RAFAEL FERNANDES PINHEIRO

THE LURIE PROBLEM AND ITS

RELATIONSHIPS WITH ARTIFICIAL NEURAL NETWORKS AND ALZHEIMER-LIKE DISEASE 

RAFAEL FERNANDES PINHEIRO

\section{THE LURIE PROBLEM AND ITS RELATIONSHIPS WITH ARTIFICIAL NEURAL NETWORKS AND ALZHEIMER-LIKE DISEASE}

Thesis presented for the degree of Doctor of Science to the Escola Politécnica of Universidade de São Paulo. 

RAFAEL FERNANDES PINHEIRO

\section{THE LURIE PROBLEM AND ITS \\ RELATIONSHIPS WITH ARTIFICIAL NEURAL NETWORKS AND ALZHEIMER-LIKE DISEASE}

Corrected Version

Thesis presented for the degree of Doctor of Science to the Escola Politécnica of Universidade de São Paulo.

Concentration Area:

Systems Engineering

Advisor:

Prof. Dr. Diego Colón 

Autorizo a reprodução e divulgação total ou parcial deste trabalho, por qualquer meio convencional ou eletrônico, para fins de estudo e pesquisa, desde que citada a fonte.

Este exemplar foi revisado e corrigido em relação à versão original, sob responsabilidade única do autor e com a anuência de seu orientador.

São Paulo, 24 de __ janeiro de 2022

Assinatura do autor:

Assinatura do orientador:

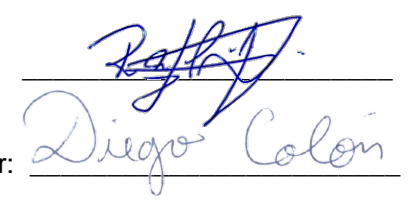

\section{Catalogação-na-publicação}

Pinheiro, Rafael Fernandes

The Lurie Problem and its Relationships with Artificial Neural Networks and Alzheimer-Like Disease / R. F. Pinheiro -- versão corr. -- São Paulo, 2022. $157 \mathrm{p}$.

Tese (Doutorado) - Escola Politécnica da Universidade de São Paulo. Departamento de Engenharia de Telecomunicações e Controle.

1.Lurie problem 2.Robust control 3.Hopfield network 4.mu-analysis 5.Alzheimer disease I.Universidade de São Paulo. Escola Politécnica. Departamento de Engenharia de Telecomunicações e Controle II.t. 



\section{Dedication}

To the One who is the $\alpha$ and the $\omega$, the Author of life, knowledge, all creation... 



\section{ACKNOWLEDGMENTS}

At the end of this journey of more than five years, it is time to thank everyone who has contributed and helped me to complete this work, where although their names do not appear on the cover, they are as much authors as I am. With this consideration, my thanks follow:

First of all to God Father, Son and Spirit. Where in the course of developing this research I have found that He can act in our lives as the $\mu$ of this work. That is, He leads us to excellent conditions of stability and performance.

To my advisor, Prof. Dr. Diego Colón, who directed me to unfailing paths in obtaining the results presented here and with his patience and wisdom formed me a scientist and researcher.

To my beloved wife Elaine, who always encouraged and supported me.

To my daughters Isabelle and Destiny where their sweetness and love inspire and sustain me.

To my parents Celso and Dorinha, and my sisters Ana Paula, Karla, and Sandra, who always supported me in all things.

To Prof. Dr. Manuel Garcia, Sonia Garcia and Pedro A. Tonelli (IME/USP), who introduced me to the incredible world of research and since my undergraduate were always present, giving precious advice and suggestions.

To Prof. Dr. Átila M. Bueno (UNESP), by the encouragement and valuable suggestions.

To everyone at LAC/USP, especially Professors Piqueira and Bruno, who transmitted me valuable knowledge. Also from LAC, to my friends André, Leo, Bruno and Andres, who were always willing to help and bring joy to our laboratory.

To Prof. Dr. Carlos Guedes and Dr. José Gaspar, who, in the final stretch of the doctorate, received me as a research fellow in the renowned CENTEC in Lisbon and supported me, where I was able to gain maturity and acquire scientific experience.

To my psychologist, counselor, and friend Marco Antônio, who helped me to overcome the most difficult moments, and to use my craziness to my advantage.

To MTVidas, Deror and Kingdom Culture. These places are really Heaven on Earth.

Finally, I thank all my friends that I have not mentioned here, but that certainly helped me by the simple fact of being my friends. 

"I am a Christian, that means: I believe in the divinity of Jesus Christ together with Tycho Brahe, Copernicus, Descartes, Newton, Fermat, Leibniz, Pascal, Grimaldi, Euler, Guldin, Boscowitsch, Gerdil, with all the great astronomers, all the great researchers of the natural sciences, all the great mathematicians of past centuries. And if you ever ask me by the reason, I will be happy to explain it. You will see that my conviction is the result of careful study and not of prejudice."

-Augustin Louis Cauchy (1789-1857), mathematician and physicist-

(GUTZWILLER, 2000) 



\section{RESUMO}

Nesta tese, são apresentados estudos que fornecem novas condições suficientes para a análise da estabilidade absoluta de sistemas do tipo Lurie para os casos Single-InputSingle-Output (SISO) e Multiple-Input-Multiple-Output (MIMO). Técnicas de projetos de controladores para sistemas tipo Lurie são deselvolvidas e aplicadas em redes neurais artificiais. Os resultados apresentados são baseados na teoria de controle $\mathcal{H}_{\infty}$, sendo, para o caso SISO, usada a técnica de sensibilidade mista (S/KS/T) e para o caso MIMO utilizada a técnica de análise e síntese $\mu$. Além disso, obtem-se condições para sistemas com atraso no tempo, mostrando que os resultados podem também ser utilizados quando existem atrasos. Na aplicação, primeiramente se faz a modelagem de uma neuropatologia que simula perda de memória usando redes de Hopfield em tempo contínuo, que é denominada neste trabalho como doença tipo-Alzheimer. Em seguida, o controlador desenvolvido, baseado na teoria deste trabalho, é aplicado para corrigir o problema de falha de memória. Exemplos são apresentados para ilustrar a teoria e simulações são realizadas para validar e mostrar a eficácia dos resultados.

Palavras-Chave - Problema de Lurie. Controle robusto. Incertezas paramétricas. Análise $\mu$. Iteração DK. Redes de Hopfield. Mal de Alzheimer. 



\section{ABSTRACT}

In this thesis, studies are presented that provide new sufficient conditions for the analysis of the absolute stability of Lurie type systems for the Single-Input-Single-Output (SISO) and Multiple-Input-Multiple-Output (MIMO) cases. From these new conditions obtained, controller designs for Lurie type systems are developed and applied to artificial neural networks. The results presented are based on the $\mathcal{H}_{\infty}$ control theory, using the mixed-sensitivity technique $(\mathrm{S} / \mathrm{KS} / \mathrm{T})$ and the $\mu$-analysis and synthesis technique. In addition, conditions for time-delay systems are also obtained. In the application, it is presented the model of a neuropathology that simulates memory loss using Hopfield networks in continuous time, which is called Alzheimer-like disease. Then, the developed controller, based on the theory of this work, is applied to correct the problem of memory failure. Examples are presented to illustrate the theory and simulations are performed to validate and show the effectiveness of the results.

Keywords -Lurie problem. Robust control. Parametric uncertainty. $\mu$-analysis. DKiteration. Hopfield network. Alzheimer disease. 



\section{LIST OF FIGURES}

1 The three axes of rotation of an aircraft. . . . . . . . . . . . . 26

2 Typical control system block diagram . . . . . . . . . . . . . . . 26

3 Aircraft control system with rudder control. . . . . . . . . . . . . . . 27

4 Block diagram of the Lurie type system. . . . . . . . . . . . . . . 27

5 Types of functions $f:$ a) $F_{(0, k]}, F_{(0, k)}$; b) $F_{\left[k_{1}, k_{2}\right]}$; c) $F_{\infty} \ldots \ldots 28$

6 Block diagram of the MIMO Lurie type system. . . . . . . . . . . . . . . . 29

7 Geometric notion of stability in dimension 2. . . . . . . . . . . . . . . 40

8 Geometric notion of asymptotic stability in dimension 2. . . . . . . . . . 40

9 Geometric notion of instability in dimension 2 . . . . . . . . . . . . . . 41

10 Block diagram of linear system. . . . . . . . . . . . . . . . . . . . . 41

11 Mapping $0<f(\sigma) \leq k \sigma \ldots \ldots \ldots \ldots \ldots$. . . . . . . . 44

12 The Popov's criterion - Theorem 1. . . . . . . . . . . . . . . 45

13 (a) $S$ as lower LFT in terms of $K . \quad$ (b) $S$ as upper LFT in terms of $\Delta .47$

$14 \Lambda$ System. . . . . . . . . . . . . . . . . . . . . . . 49

$15 \quad \Lambda$ - $\Delta$-Structure. . . . . . . . . . . . . . . . . . . . . . . . . 49

16 Mapping $0<f_{j}\left(\sigma_{j}\right) \leq k_{j} \sigma_{j} \ldots \ldots \ldots \ldots \ldots \ldots \ldots$

17 Perturbed transfer function. . . . . . . . . . . . . . . . . 53

18 System $L_{p}$ in closed loop. . . . . . . . . . . . . . . . . . . . 53

19 Complete diagram with all weights. . . . . . . . . . . . . . . . 55

20 Lurie type system in discrete form. . . . . . . . . . . . . . 56

21 Lurie type system in closed-loop with controller. . . . . . . . . . . . . . . 61

22 General Control System. . . . . . . . . . . . . . . . . . 61

$23 \quad M-\Delta$-Structure. . . . . . . . . . . . . . . . . 62 
$24 \quad M_{22}-\Delta$-Structure for robust stability analysis. . . . . . . . . . . . 63

$25 M-\Delta-\bar{\Delta}$-Structure for robust performance analysis. . . . . . . . . . . . . 64

26 Uncertain delay system. . . . . . . . . . . . . . . . . . . 66

27 Frequency response of $\gamma_{i}$ and $\gamma_{i, j}^{*} \ldots \ldots \ldots \ldots \ldots$

28 Equivalent systems by Lemma $3 . \ldots \ldots$. . . . . . . . . . . . . 75

29 Conditon $\left|\frac{\frac{k}{2} G_{o}(j w)}{1+G_{o}(j w)}\right|<1, \forall \omega$ satisfied for $\mathrm{k}=0.8677 \ldots \ldots \ldots$. . . . . . . 78

30 Popov's method for $k=0.8662 \ldots \ldots \ldots \ldots$. . . . . . . . . . 79

31 Block diagram of the Lurie type system with delay. . . . . . . . . . . . 79

32 Simplified block diagram. . . . . . . . . . . . . . . . . 80

33 Chua's Circuit unstable with $\tau=0.8 \ldots \ldots \ldots$. . . . . . . . 82

34 Condition (3.11) satisfied for $\tau_{\max }=0.4206$ visualized via Bode diagram. $\quad 82$

35 Absolute Stability to Chua's Circuit with $\tau=0.4206$ via Simulink ${ }^{\circledR} \ldots$. . . 83

36 Response to sine wave. . . . . . . . . . . . . . . 86

37 Response to step signal. . . . . . . . . . . . . . . . . . . 87

38 Block diagram of the numerical example. . . . . . . . . . . . . . . . 87

39 Step response of the family of plants with controller. . . . . . . . . . . . 89

40 The hyperbolic tangent. . . . . . . . . . . . . . . . . . . 89

41 Response to sine wave with discrete controller by mixed-sensitivity $(S / K S)$ with continuous plants. . . . . . . . . . . . . . . . . 90

42 Response to pulse wave with discrete controller by mixed-sensitivity $(S / K S)$ with continuous plants. . . . . . . . . . . . . . . . . 9 90

43 Step response of the family of plants with controller $(\mathrm{S} / \mathrm{KS} / \mathrm{T}) . \quad \ldots . . . \quad 91$

44 Response to sine wave with discrete controller by mixed-sensitivity $(S / K S / T)$ with continuous plants. . . . . . . . . . . . . . . . . . . 92

45 Response to pulse wave with discrete controller by mixed-sensitivity $(S / K S / T)$ with continuous plants. . . . . . . . . . . . . . . . . . 92

46 Graphics obtained using all the controllers designed via LMI and polytopic uncertainties, and without controller $(\mathrm{w} / \mathrm{o}) . \ldots . \ldots . \ldots 93$ 
$\mu$-analysis for an absolutely stable Lurie type system. . . . . . . . . . . 100

48 Neural network of Example 6. . . . . . . . . . . . . . . . . . . 102

49 Nonlinearity. . . . . . . . . . . . . . . . . . . . . . . . 103

$50 \mu$-analysis with sectors $0<f\left(x_{1}\right) \leq k_{1} x_{1}$ and $0<f\left(x_{2}\right) \leq k_{2} x_{2}\left(k_{1}=k_{2}=2\right) .103$

51 Check if $H(j w)>0$ for Theorem 3. . . . . . . . . . . . . . . . 104

$52 \mu$-analysis with $k_{1}=1.0999$ and $k_{2}=1.1666 \ldots \ldots \ldots 4$

$53 \quad \Lambda$ - $\Delta$-D-Structure . . . . . . . . . . . . . . . . . . . . 108

54 Chua's circuit oscillations. . . . . . . . . . . . . . . . . 112

$55 \quad \mu$-analisys. . . . . . . . . . . . . . . . . . 113

56 Absolute stability to Chua's circuit. . . . . . . . . . . . . . 113

$57 \Lambda-\Delta-\bar{\Delta}$-Structure in closed-loop with controller. . . . . . . . . . . . . 121

58 Neural network without time-delay. . . . . . . . . . . . . . . . 123

59 Neural network with time-delay. . . . . . . . . . . . . . . . . . 123

$60 \mu$-analysis of stability. . . . . . . . . . . . . . . . . 124

61 Temporal response without control. . . . . . . . . . . . . . . 125

62 Temporal response with control. . . . . . . . . . . . . . . . . 125

63 Time-delayed without control. . . . . . . . . . . . . . 125

64 Time-delayed with control. . . . . . . . . . . . . . . . 125

65 Hopfield's stability. . . . . . . . . . . . . . . . . . . . . . 129

66 Network architecture to simulate a $3 \times 4$ digital sign. . . . . . . . . . . . 130

67 Synapse between neurons. . . . . . . . . . . . . . . . . . 131

68 Hopfield's network via Simulink ${ }^{\circledR} \ldots \ldots \ldots$. . . . . . . . . . . . . . . . 132

69 Memory loss modeling process. . . . . . . . . . . . . . . . . . 132

70 Healthy network temporal response. . . . . . . . . . . . . . . . . . 133

71 Temporal evolution of the memory of the letter L via digital sign. . . . . . 134

72 Temporal response for network with partial memory loss. . . . . . . . . . . 134 
73 Temporal evolution of the letter L for network with partial loss of memory via digital sign. . . . . . . . . . . . . . . . . . . . . . 135

74 Temporal response of the worst case of memory loss. . . . . . . . . . . 135

75 Complete loss of memory via digital sign. . . . . . . . . . . . . . . . . 136

76 Hopfield's network with controllers. . . . . . . . . . . . . . . 136

77 Condition (4.31) not satisfied. . . . . . . . . . . . . . . 137

78 Value of $\mu$ that satisfies the condition (4.31) of Theorem 25. . . . . . 138

79 Temporal response of the network with controllers. . . . . . . . . . 138

80 Temporal evolution of the letter L in digital sign for network controlled. . . 139 


\section{LIST OF TABLES}

1 Works published. . . . . . . . . . . . . . . 34

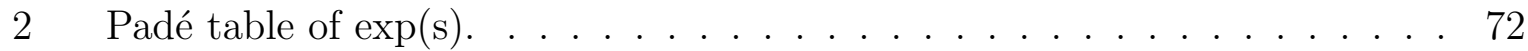

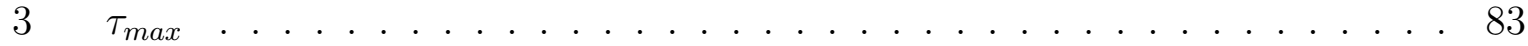

$4 \tau_{\max }$ and Padé approximation order $\ldots \ldots \ldots \ldots$

5 Controller obtained using Theorem 5 for $l \in[2,3,4] \ldots \ldots$. . . . . . . 93

$6 \quad \tau_{\max }$ and Padé approximation order. . . . . . . . . . . . . . 112

$7 \quad \tau_{\max }$ in different theorems. . . . . . . . . . . . . . 112 



\section{CONTENTS}

1 Introduction $\quad 25$

1.1 Lurie Problem in SISO Case . . . . . . . . . . . . . . . . 27

1.2 Lurie Problem in MIMO Case . . . . . . . . . . . . . . . . . . . . 28

1.3 Related Work . . . . . . . . . . . . . . . . . . . . . . 30

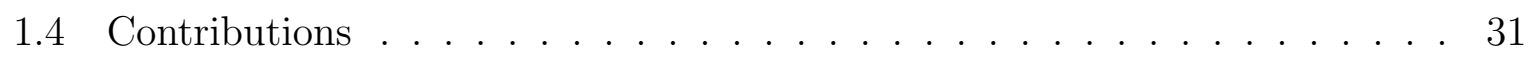

1.4.1 Contributions in Continuous SISO Systems . . . . . . . . . . . . . . 31

1.4.2 Contributions in Discrete SISO Systems . . . . . . . . . . . . . 32

1.4.3 Contributions in Continuous MIMO Systems . . . . . . . . . . . . . 32

1.4.4 Works Published ....................... 34

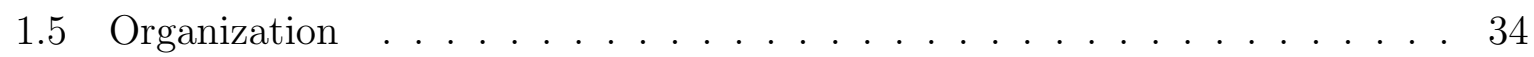

2 Theoretical Background $\quad 37$

2.1 Basic Definitions . . . . . . . . . . . . . . . 37

2.1 .1 Norms . . . . . . . . . . . . . . . . . 37

2.1.1.1 Vector Norm . . . . . . . . . . . . 37

2.1.1.2 Matrix Norm . . . . . . . . . . . . . . 38

2.1.2 Stability Definitions . . . . . . . . . . . . . . 39

2.1.2.1 Stability in the Sense of Lyapunov . . . . . . . . . . . . 39

2.1.2.2 Absolute Stability . . . . . . . . . . . . . . 42

2.1.2.3 Robust Stability . . . . . . . . . . . . . . . . 42

2.2 Three Theorems for Absolute Stability . . . . . . . . . . . . . . . 44

2.2.1 Popov Criterion . . . . . . . . . . . . . . . . . . 44

2.2.2 Theorem of Explicit Condition . . . . . . . . . . . . . . . 45

2.2.3 Generalized Popov's Criterion . . . . . . . . . . . . . . . 46 
2.3 Linear Fractional Transformations . . . . . . . . . . . . . . . . . . . . . . 46

2.3.1 Linear State-Space System with Normalized Uncertainties . . . . . 48

2.4 Modeling Nonlinearity by Means of Parametric Uncertainties and LFT . . 49

2.4.1 SISO Case . . . . . . . . . . . . . . . . . . . . . 49

2.4 .2 MIMO Case . . . . . . . . . . . . . . . . . . . 50

2.4.3 Lurie type System via Parametric Unicertainty and LFT . . . . . . 52

2.5 Mixed-Sensitivity Method . . . . . . . . . . . . . . . . 52

2.5.1 Discretization Procedure with Parametric Uncertainties . . . . . . 55

$2.6\|\mathcal{H}\|_{\infty}$ State Feedback Controller Design via LMI Method . . . . . . . . . 56

2.6.1 Discretization with Polytopic Uncertainties . . . . . . . . . . 58

$2.7 \mu$-Analysis and Synthesis . . . . . . . . . . . . . . . . . 60

2.7.1 General Control Formulation with Uncertainties . . . . . . . . . . 60

2.7.2 Robustness of Stability via $\mu \ldots \ldots$. . . . . . . . . 61

2.7.3 Robustness of Performance via $\mu \ldots \ldots$. . . . . . . . . 64

$2.7 .4 \mu$-Analysis for Systems with Delay . . . . . . . . . . . . . 64

2.7.5 $\mu$-Synthesis via DK-Iteration . . . . . . . . . . . . . . . . 67

2.8 Padé Approximation . . . . . . . . . . . . . . . . . . . . . 69

2.8.1 Classical Formulation of Padé Approximation _ . . . . . . . . . 69

2.8.2 Modern Formulation of the Padé Approximation . . . . . . . . . . 70

2.8.3 Padé Approximation of the Exponential Function . . . . . . . . . . 71

2.8.4 Theorems Useful for Padé Approximation . . . . . . . . . . . . . 72

2.8.5 Some Concepts for Rational Matrices and Complex Functions . . . 73

3 Sufficient Conditions in SISO Case $\quad 75$

3.1 Sufficient Conditions . . . . . . . . . . . . . . . . . 75

3.2 Time-delay SISO Lurie type systems . . . . . . . . . . . . . . . . . . . . . 79

3.3 Synthesis of the Controller in Continuous Case . . . . . . . . . . . . . 83 
3.4 Approach in the Discrete Case . . . . . . . . . . . . . . . . . 87

4 Sufficient Conditions in MIMO Case $\quad 95$

4.1 Sufficient Conditions . . . . . . . . . . . . . . . . . . 95

4.2 Time-Delay MIMO Lurie Type Systems . . . . . . . . . . . . . . . . . . 106

4.3 Proof of the Theorem $21 \ldots \ldots \ldots \ldots$. . . . . . . . . . . . . . . . . . .

4.4 Controller Synthesis _. . . . . . . . . . . . . . . . . . . 120

$\begin{array}{llr}5 & \text { Application } & 127\end{array}$

5.1 Alzheimer's Disease . . . . . . . . . . . . . . . . . . . . 128

5.2 Hopfield's Network and its Relationship with Lurie Problem . . . . . . . . . . . . . . . . . . . . . 128

5.3 Modeling Memory Loss Due to Alzheimer-Like's Disease via Hopfield Networks . . . . . . . . . . . . . . . . . . . 130

5.3 .1 Simulation . . . . . . . . . . . . . . . . 132

5.4 Application of a Result of Lurie Problem in Hopfield's Network . . . . . . 135

5.4 .1 Simulation . . . . . . . . . . . . . . . 136

6 Conclusion $\quad 141$

6.1 Discussion and Summary of the Results . . . . . . . . . . . . . . . . . 141

6.2 Suggestions for Future Research . . . . . . . . . . . . . . . . . . . . 143

References 



\section{INTRODUCTION}

The Lurie problem (LP), or absolute stability problem, proposed by the mathematician A.I. Lurie in the mid-1940s, came to light due to an aircraft automatic control problem (LURIE; POSTNIKOV, 1944). It consists in finding necessary and sufficient conditions on the parameters of the system in Figure 4 or Figure 6, such that the stability of the global equilibrium point is guaranteed. Although no necessary and sufficient conditions were found for the general MIMO case, the study of this problem has left significant contributions in mathematics and engineering, specially in subareas such as theory of stability and control, and laid the foundation for a new and important area: robust control.

Anatoly Isakovich Lurie (July 19, 1901 - February 12, 1980) was born in Mogilev (Belarus), and worked in the field of mechanics and control theory, leaving a great legacy. Lurie type systems and their nonlinearities can reflect to physical, mechanical, biological, and other various types of dynamical systems. Based on this system and the analysis of the stability of the null solution of the system (that is the equilibrium point), one could get a control law, seeking necessary and sufficient conditions to global asymptotic stability of the system.

Fixed-wing aircrafts have moving surfaces in order to control the flight, that are: the elevators, ailerons, and rudder. The elevators are responsible for the rise or descent movement of the aircraft (pitch), the ailerons for rolling and rudders for direction movements (yaw) of the aircraft, as presented in Figure 1.

In control theory, the basic structure of a control system is generally based on the feedback, as presented in Figure 2, where $\bar{G}$ and $K$ are linear systems, represented by transfer functions. $\bar{G}$ models the part to be controlled (the plant) and $K$ represents mathematically the controller. According to (OGATA, 2010) a transfer function is a mathematical model which is an operational method to express the ordinary differential equation that relates the output variable and the input variable. 
Figure 1: The three axes of rotation of an aircraft.

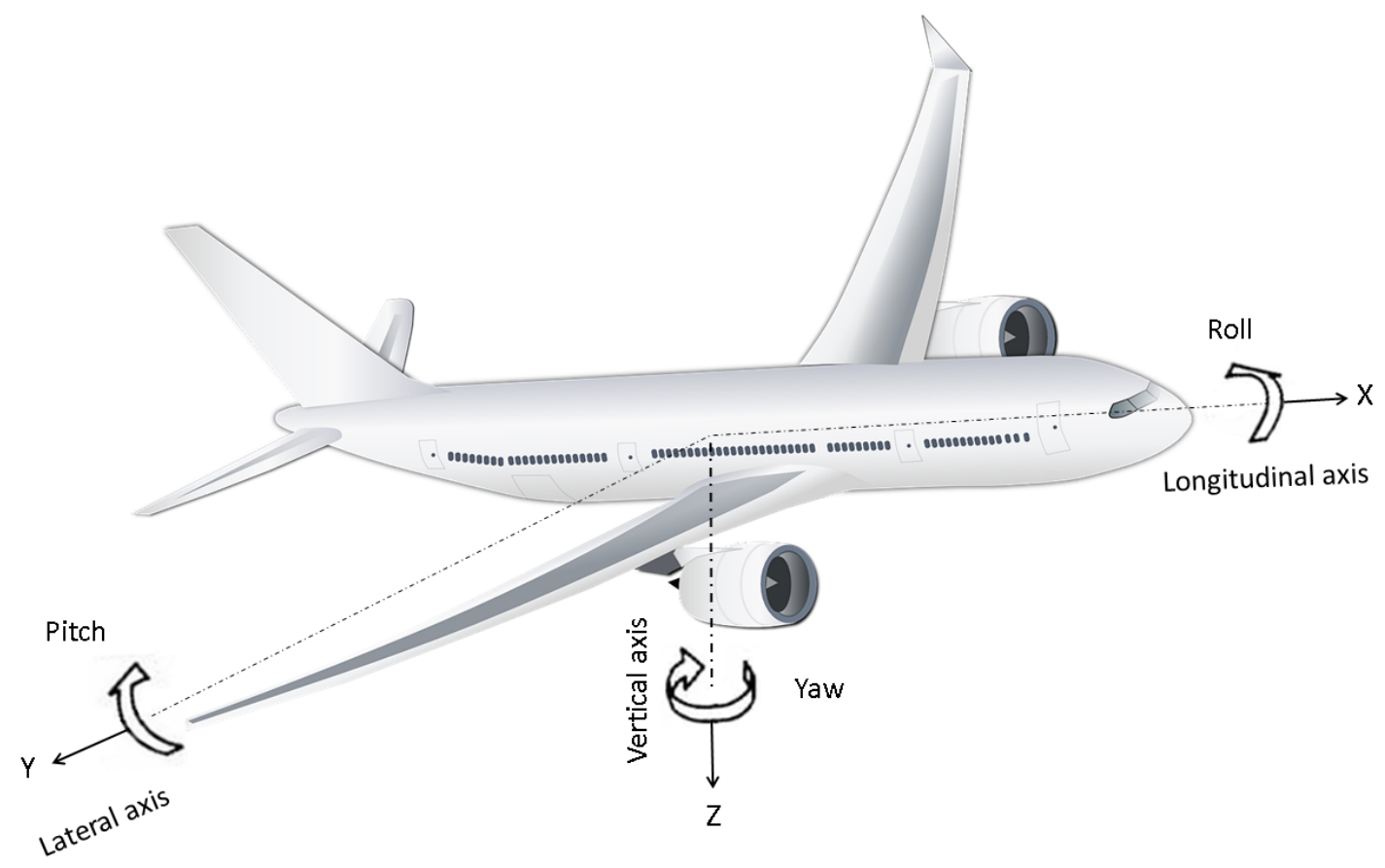

Source: https://stock.adobe.com/

Figure 2: Typical control system block diagram

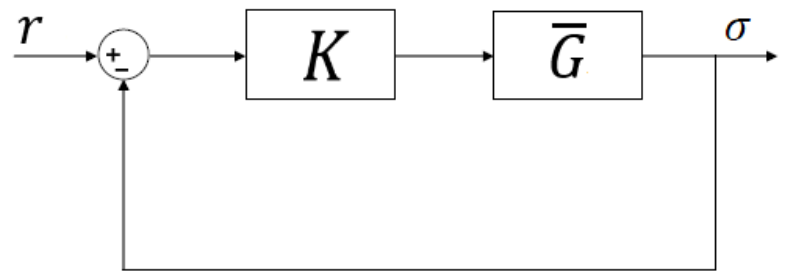

Source: Author.

In an aircraft, there are lots of feedback loops, like in Figure 2. Some of then can be performed by the pilot, as for example, to check whether the real route of the aircraft $\sigma$ matches the desired route $r$.

On the other hand, this task could also be performed by the autopilot system, which has an input signal $r$ that is the direction selected by the pilot. Usually this input signal is related to the frequency of a land station $\mathrm{NDB}, \mathrm{VOR}^{1}$ or even a radio station. This direction is constantly compared with the actual route of the aircraft $\sigma$ and if there is any deviation, then control $K$ runs the position correction, through the acting on the control surface.

\footnotetext{
${ }^{1} \mathrm{NDB}$ or VOR are earth stations that send signals of radiofrequency of reference for aircraft navigation
} 
Figure 3: Aircraft control system with rudder control.

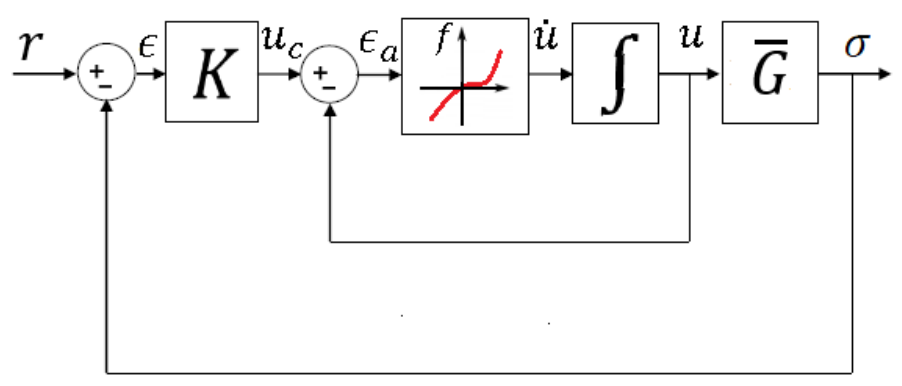

Source: Author.

However, some control loops deviates significantly from the linear ones, as for example, the one shown in Figure 3. It has been found that automatic control loops in aircraft that use control surfaces have nonlinearities, as for example, saturation of the actuator. Considering that $u$ represents the deflection of the rudder, the rudder control is intended to keep the deflection $u_{c}$ fixed by the controller $K$ until the aircraft direction deviation $\epsilon$ is corrected. What Lurie did was to separate the linear part of the nonlinear part of the model's differential equations (calculations can be checked in (RASVAN, 2011)).

\subsection{Lurie Problem in SISO Case}

In Figure 4, it is shown a general form of this separation, where the linear dynamics is represented by the system $G$, and the function $f(\sigma)$ in the feedback, represents the nonlinearity.

Figure 4: Block diagram of the Lurie type system.

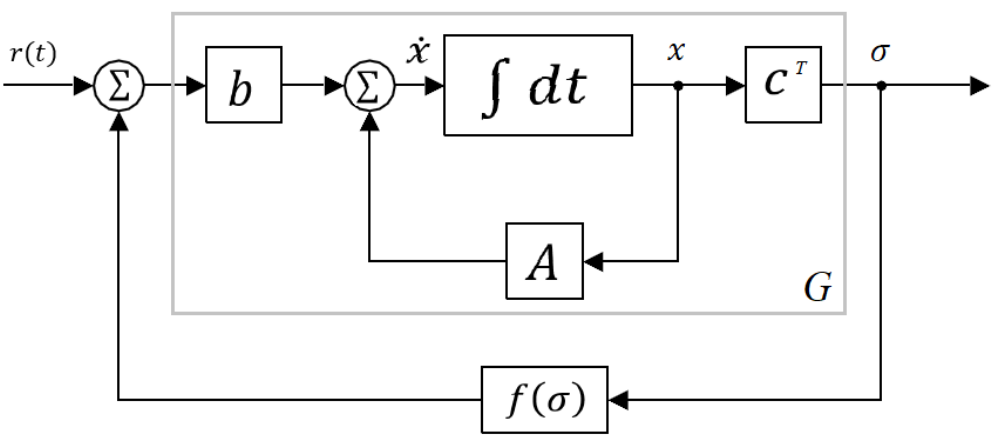

Source: Author.

The LP in SISO case, or absolute stability problem, consists in finding necessary and sufficient conditions to the global asymptotic stability of the system in Figure 4. Considering $r=0$, the diagram in Figure 4 is expressed by the system of differential 
equations presented in (1.1), which is known in the literature as a Lurie type system:

$$
\left\{\begin{array}{l}
\dot{x}=A x-b f(\sigma), \\
\sigma=c^{T} x
\end{array}\right.
$$

where $x \in \mathbb{R}^{n}$ is the state vector and $b, c \in \mathbb{R}^{n}, A \in \mathbb{R}^{n x n}$ are fixed matrices. Generally, the nonlinearity $f(\sigma)$ is a continuous function restricted to the first and third quadrants of the plane (see Figure 5). It belongs to one of the following families:

$$
\begin{gathered}
F_{(0, k]}:=\left\{f \mid f(0)=0,0<\sigma f(\sigma) \leq k \sigma^{2}, \sigma \neq 0\right\}, \\
F_{(0, k)}:=\left\{f \mid f(0)=0,0<\sigma f(\sigma)<k \sigma^{2}, \sigma \neq 0\right\}, \\
F_{\left[k_{1}, k_{2}\right]}:=\left\{f \mid f(0)=0, k_{1} \sigma^{2} \leq \sigma f(\sigma) \leq k_{2} \sigma^{2}, \sigma \neq 0\right\}, \\
F_{\infty}:=\{f \mid f(0)=0, \sigma f(\sigma)>0, \sigma \neq 0\} .
\end{gathered}
$$

Figure 5: Types of functions $f:$ a) $F_{(0, k]}, F_{(0, k)}$; b) $F_{\left[k_{1}, k_{2}\right]}$; c) $F_{\infty}$.

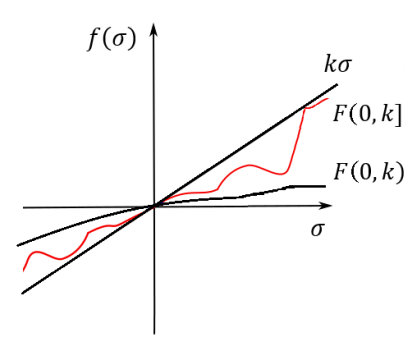

(a)

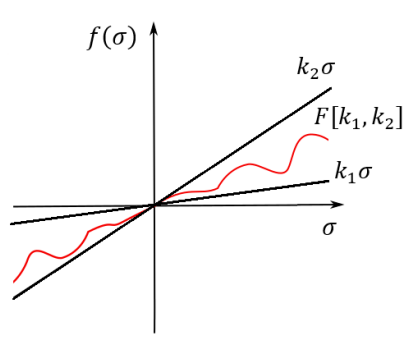

(b)

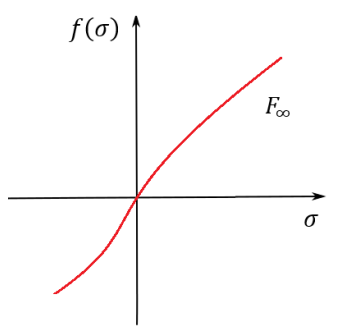

(c)

Source: Author.

\subsection{Lurie Problem in MIMO Case}

Figure 6 shows the Lurie type system block diagram in the MIMO case. In this case, there are more than one nonlinearity $f_{j}\left(\sigma_{j}\right)$, becoming a multiple input problem.

The diagram in Figure 6 is expressed by the system of differential equations in (1.2): 
Figure 6: Block diagram of the MIMO Lurie type system.

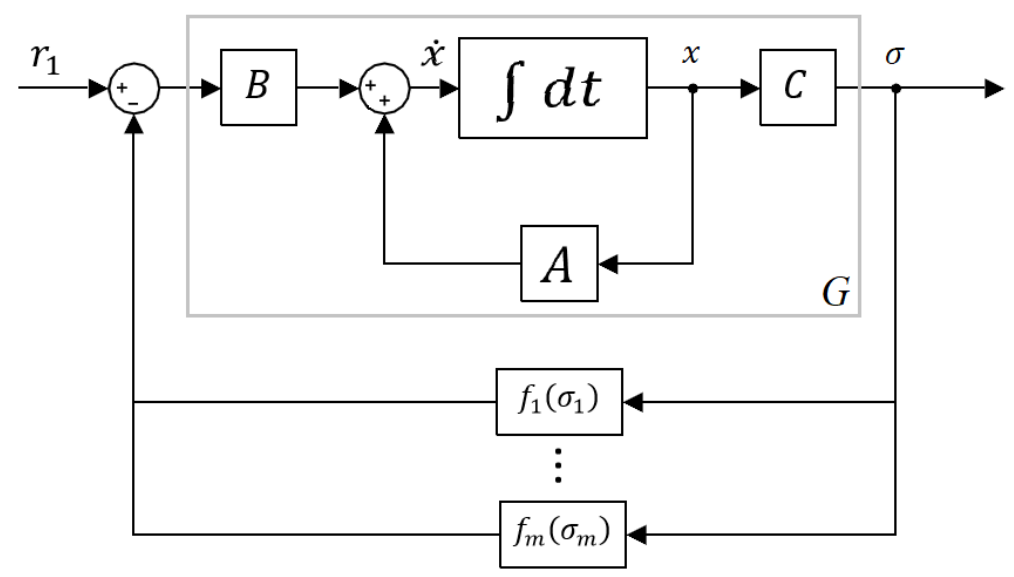

Source: Author.

$$
\left\{\begin{array}{l}
\dot{x}=A x-B f(\sigma)+B r_{1}, \\
\sigma=C x
\end{array}\right.
$$

where $x \in \mathbb{R}^{n}$ is the state vector, $f=\left[f_{1}, f_{2}, \cdots, f_{m}\right] \in \mathbb{R}^{m}$ is a vector of unknown but fixed functions and $\sigma \in \mathbb{R}^{m}$. Also the matrices $B \in \mathbb{R}^{n \times m}, C \in \mathbb{R}^{m \times n}$ are known and fixed and $A \in \mathbb{R}^{n \times n}$ is Hurwitz, know and fixed.

In general, the nonlinear functions $f_{j}\left(\sigma_{j}\right)$ are continuous and restricted to the first and third quadrants of the plane. They may belong to one of the following families, where $k$ is the sector that limits $f_{j}\left(\sigma_{j}\right)$ :

$$
\begin{gathered}
F_{\left(0, k_{j}\right]}:=\left\{f_{j} \mid f_{j}(0)=0,0<\sigma_{j} f_{j}\left(\sigma_{j}\right) \leq k \sigma_{j}^{2}, \sigma_{j} \neq 0\right\}, \\
F_{\left(0, k_{j}\right)}:=\left\{f_{j} \mid f_{j}(0)=0,0<\sigma_{j} f_{j}\left(\sigma_{j}\right)<k \sigma_{j}^{2}, \sigma_{j} \neq 0\right\}, \\
F_{\left[\underline{k}_{j}, \bar{k}_{j}\right]}:=\left\{f_{j} \mid f_{j}(0)=0, \underline{k}_{j} \sigma_{j}^{2} \leq \sigma_{j} f_{j}\left(\sigma_{j}\right) \leq \bar{k}_{j} \sigma_{j}^{2}, \sigma_{j} \neq 0\right\}, \\
F_{\infty}:=\left\{f_{j} \mid f_{j}(0)=0, \sigma_{j} f_{j}\left(\sigma_{j}\right)>0, \sigma_{j} \neq 0\right\} .
\end{gathered}
$$

It can be assumed, without loss of generality, that the set of line vectors $c_{i}=$ $\left(c_{i 1}, \ldots, c_{i n}\right), i=1,2, \ldots, m$ of matrix $C$, are linearly independent, and with the purpose of separating the variables, by a transformation (see (LIAO; YU, 2008)) the system (1.2) can be transformed in the equations in (1.3) or (1.4):

$$
\dot{y}=\tilde{A} y+\sum_{j=n-m+1}^{n} \tilde{b}_{j} \tilde{f}_{j}\left(y_{j}\right),
$$




$$
\dot{y}_{i}=\sum_{j=1}^{n} \tilde{a}_{i j} y_{j}+\sum_{j=n-m+1}^{n} \tilde{b}_{i j} \tilde{f}_{j}\left(y_{j}\right) .
$$

\subsection{Related Work}

Many researchers have initially worked on this problem in the 1950s and 1960s, among whom one can mention Aizerman (AIZERMAN, 1947), with his well known conjecture (proved wrong afterwards), Krasovskii (KRASOVSKII, 1953), Popov (POPOV, 1961), and Kalman (KALMAN, 1963). The research in the LP has grown since 1980s, when different papers were published linking this problem to other areas and approaches, such as neural networks (FORTI et al., 1994; KASZKUREWICZ; BHAYA, 1995; PINHEIRO; COLÓN, 2019; TOWNLEY et al., 2000; YI; HENG; VADAKKEPAT, 2002); chaos, Chua's circuits and Hopfield neural network (HNN) (LIAO; YU, 2008; KAZEMY; FARROKHI, 2017; PINHEIRO, 2015; LIN; WANG; TAN, 2020); convex approach (GAPSKI; GEROMEL, 1994); active disturbance rejection control (QI; XIA; AL., 2017); $\mu$-analysis (ABTAHI; YAZDI, 2019; BERNSTEIN; HADDAD; SPARKS, 1995; LEE; JUANG, 2005); Linear Fractional Transformation (LFT) (ALVERGUE; GU; ACHARYA, 2013); uncertain systems (TAN; ATHERTON, 2003); Integral Quadratic Constraints (IQC) and ZamesFalb multipliers (CARRASCO; TURNER; HEATH, 2016); and Linear Parameter Varying (LPV) systems (SERON; DONA, 2015; YU; LIAO, 2019). In addition to all these new lines of applications, its study remains current in the aeronautical field, as can be seen in (IMANI, 2019).

In order to solve this problem, Nyquist criterion, Lyapunov functions and Linear Matrix Inequalities (LMI) are used. For example, the Circle criterion (ZAMES, 1966) uses the approach of Nyquist; and Popov criterion (POPOV, 1961), generally less conservative than the Circle criterion, uses Lyapunov functions in its proof which are sufficient conditions for the Single-Input-Single-Output (SISO) case. More recent works, like (DUAN et al., 2018; GAO; DU; LIU, 2017; KAZEMY; FARROKHI, 2017; QI; XIA; AL., 2017; YU; LIAO, 2019) also use Lyapunov functions and LMI. In addition, works have emerged that revisit past problems in order to bring new conditions for absolute stability, for example, in (NADERI et al., 2019) the conjectures of Aizerman and Kalman to provide new criteria for absolute stability are addressed. In SISO case others works also have emerged, as (LI et al., 2018), where new conditions for instability are presented.

Multivariate versions of the circle and Popov criteria have been formulated, which also 
present only sufficient conditions for absolute stability (VIDYASAGAR, 1993; HADDAD; BERNSTEIN, 1993). Many other papers have also provided sufficient conditions for several cases of this problem that also use the Lyapunov techniques, as for example: conditions for Lurie systems with parameter intervals (LIAO et al., 2007), systems with delays (HAO; ZHAO, 2010; GAO; DU; LIU, 2017) and chaotic systems (ZHENG; CUI, 2018). Several examples of stabilizing controllers design are mentioned in (PEIRAN et al., 2012; WANG; XUE; ZHANG, 2016; GYURKOVICS; ESZES, 2015; ZENG et al., 2014; ZENG et al., 2010). However, there still remains a need for more general results that that establish necessary and sufficient conditions for absolute stability and controllers designs in MIMO cases.

The $\mu$-analysis and synthesis techniques were introduced in classical works due to Doyle (DOYLE, 1982), Skogestad (SKOGESTAD; POSTLETHWAITE, 2007) and Zhou (ZHOU; DOYLE; GLOVER, 1995). They use, as the main tool, the Structured Singular Value (SSV), also known as $\mu$, which must be calculated in the frequency domain and for different frequencies, in order to guarantee robustness of stability and performance for closed-loop systems. The SSV is a very powerful tool for determining the robustness of stability and performance, as it comes from the Nyquist criterion. Recent works have arisen using Doyle's techniques (SANJEEWA; PARNICHKUN, 2019; GAO; YANG; SHARDT, 2018). On the other hand, its calculation can be done only in computers, as it involves complex optimization problems. There are commercial software capable of doing them, as for example Matlab ${ }^{\circledR}$. In addition, the SSV can be used in the synthesis of controllers, also known in the literature as $\mu$-synthesis or DK-Iteration. Besides $\mu$, the design of controllers via mixed-sensitivity, also introduced by Doyle, is a technique also used for the development of absolute stability conditions and design of controller of this work.

\subsection{Contributions}

The following is a summary of contributions of this work.

\subsubsection{Contributions in Continuous SISO Systems}

Based in the idea of controller design using $\mathcal{H}_{\infty}$ Mixed-Sensitivity (S/KS/T), two new conditions in frequency domain to absolute stability of the Lurie type systems are presented in Theorems 13 and 14, whose proof are original from this author (also Lemma 
2, which is necessary to this proof). The level of conservatism is similar to the Popov's criterion. Furthermore, from the Theorem 14, a new condition is obtained in Theorem 15 for time-delay system and applied in Chua's circuits. This theorem is the proof of the conjecture of (PINHEIRO; COLÓN, 2021a). The new condition is showed to be better than other conditions in the literature. In Theorems 16 and 17, the design of controllers via $\mathcal{H}_{\infty}$ Mixed-Sensitivity $(\mathrm{S} / \mathrm{KS} / \mathrm{T})$ is generalized to any SISO Lurie type system, as it was done in (PINHEIRO; COLÓN, 2017) for a specific system. Thereby, the problem of performance and robustness of performance of the closed loop Lurie type system in SISO case can be solved. Some of these results were published in (PINHEIRO; COLÓN, 2017) and (PINHEIRO; COLÓN, 2021a).

\subsubsection{Contributions in Discrete SISO Systems}

Two methodologies are presented in the discrete-time domain version. The first, as made in (PINHEIRO; COLÓN, 2017) in continuous-time domain, a technique of design of controllers via mixed-sensitivity $\mathcal{H}_{\infty}$ for Lurie type systems with parametric uncertainties is developed. In the second, the design of controller is made via LMI and polytopic uncertainties, with the theoretical basis for this approach obtained in works like: (SHIEH; WANG; CHEN, 1998; OLIVEIRA; GEROMEL; BERNUSSOU, 2002; HETEL; DAAFOUZ; IUNG, 2007; GEROMEL; KOROGUI, 2011); and (BRAGA et al., 2013). These results are being prepared for submission to a relevant journal in the area.

\subsubsection{Contributions in Continuous MIMO Systems}

It is presented a new technique using $\mu$-analysis and LFT, which applies not only to the absolute stability analysis, but also to the design of controllers for Lurie type systems. The results obtained for the stability analysis guarantee sufficient conditions for systems without time-delay. Another contribution of this work is a solution of the problem of performance and robustness of performance for the closed-loop system for MIMO cases, as it has been done in (PINHEIRO; COLÓN, 2017) for SISO case. Furthermore, all these results also enable the synthesis of controllers via DK-iteration, which is another contribution of this work. More specifically, one of the main contributions of this work to the MIMO case is the Lemma 4, in which one proves the structures of the matrices $\Delta$ and $\Lambda$ for Lurie type systems, as well as compute them. The challenges and difficulties to get this lemma involved a lot of algebraic manipulations, which is necessary to $\mu$-analysis and synthesis. Theorems 18, 19 and 25 are also contributions, as they are extensions of 
theorems in the literature for the Lurie type systems. It is also presented examples of numerical applications of the developed theoretical results, which are in the Examples 5 to 8. Those examples are used in order to validate the results, and in the specific case of Example 6, one applies Theorem 18 to a Hopfield neural network (HNN), and finds that the developed method is as good as a famous result in the literature, which is the generalized Popov theorem (Theorem 3). On the other hand, this method is for a more general class of systems and needs less conditions to verify. For MIMO Lurie type system with delay, first it is presented Theorem 20, which is an extension of the Theorem 9, and the Theorem 21 which brings the proof of the Conjecture of (PINHEIRO; COLÓN, 2021b). This work presents contributions in the study of the convergence of the Padé approximation used in Lurie type systems and in $\mu$-analysis. These contributions are: Lemmas 5, 6, 7, 8, 9, 10; Theorems 22, 23, 24; and Corollary 1. The Example 7 is the application of Theorem 21 for a well know problem in the literature, that is the Chua's circuit with time-delay. It is obtained a less conservative result than other presented the literature. Finally, Example 8 is the application of Theorem 25 for controller design for systems with and without time-delay. Some of these results were published in (PINHEIRO; COLÓN, 2021b).

In the bibliographic research of this work, it was found papers that relate in some way HNN to Alzheimer Disease (AD) (MORRISON; MAIA; KUTZ, 2017; SERGIO; BRAGA; BUARQUE, 2009; SWIETLIK et al., 2020; THURAISINGHAM, 2015; TABEKOUENG; KENGNE; FOTSIN, 2020) and papers that connect LP with HNN (AOUITI; LI; MIAADI, 2019; KASZKUREWICZ; BHAYA, 1995; LIAO; YU, 2008; PINHEIRO; COLÓN, 2019). But, it was not found works that relate the LP, HNN and AD. In this sense, this author believes that it is also a contribution of this work, that is, the relationship of those three areas of research. Thus, using HNN, it is present a modeling of one of the consequences of $\mathrm{AD}$, which is memory loss, as suggested in (PINHEIRO; COLÓN, 2019; PINHEIRO; COLÓN, 2021b). Although the use of HNN in AD modeling is not new (SERGIO; BRAGA; BUARQUE, 2009; THURAISINGHAM, 2015), here it is done in a different way. It is done in continuous-time based on external inputs (like in (ZHOU et al., 2016)), instead of in discrete time domain. Other differences are: in paper (SERGIO; BRAGA; BUARQUE, 2009) the HNN is used to train the Venn's networks which models the AD; in (THURAISINGHAM, 2015) the HNN is used in conjunction with a mean field theory; in (ZHOU et al., 2016) the model is based on external inputs, but it does not use HNN. Therefore, the modeling of neuropathology in the present thesis can be considered as a contribution. Another contribution of this work is in the application 
of the recent result presented by (PINHEIRO; COLÓN, 2021b), which is the design of a controller via DK-iteration to correct the effect of the memory loss in the computationally modeled disease. In practical terms, there is still a certain difficulty in accessing the brain neurons. But with all technological development, as already seen in works such as (MORRISON; MAIA; KUTZ, 2017), it is promising that in the near future, areas of electrical engineering, such as artificial neural networks and artificial intelligence will bring even more efficient solutions for the diagnosis, treatment and cure of AD. These results were published in Congresso Brasileiro de Automática (CBA) 2020.

\subsubsection{Works Published}

During the doctoral research, until the present moment, some papers have been published (see Table 1).

Table 1: Works published.

\begin{tabular}{|c|l|l|l|l|}
\hline N. & Work's Name & Type & Event / Journal & Year \\
\hline \hline 1 & $\begin{array}{l}\text { An Application of the Lurie Lroblem in } \\
\text { Hopfield Neural Networks }\end{array}$ & Paper & DINAME & 2017 \\
\hline 2 & $\begin{array}{l}\text { Controller by } \mathcal{H}_{\infty} \text { Mixed-Sensitivity } \\
\text { Design }(\mathrm{S} / \mathrm{KS} / \mathrm{T}) \text { for Lurie Type Sys- } \\
\text { tems }\end{array}$ & Paper & COBEM & 2017 \\
\hline 3 & $\begin{array}{l}\text { An Application of the Lurie problem in } \\
\text { Hopfield Neural Networks }\end{array}$ & $\begin{array}{l}\text { Book } \\
\text { Chapter }\end{array}$ & SPRINGER & 2019 \\
\hline 4 & $\begin{array}{l}\text { On the } \mu \text {-Analysis and Synthesis of } \\
\text { MIMO Lurie type Systems with Appli- } \\
\text { cation in Complex Networks }\end{array}$ & $\begin{array}{l}\text { Paper } \\
\text { Signal Process }\end{array}$ & 2020 \\
\hline 5 & $\begin{array}{l}\text { Relating Lurie's Problem, Hopfield's } \\
\text { Network and Alzheimer's Disease }\end{array}$ & $\begin{array}{l}\text { Paper } \\
\text { Analysis and Synthesis of SISO } \\
\text { Lurie Type Systems via } \mathcal{H}_{\infty} \text { Mixed- } \\
\text { Sensitivity }\end{array}$ & $\begin{array}{l}\text { CBA } \\
\text { Paper Measur and } \\
\text { Control }\end{array}$ & 2020 \\
\hline
\end{tabular}

Source: Author.

\subsection{Organization}

This work is organized as follow: in Chapter 2, important theorems and definitions are presented, which can be found in the literature and form the basis of the developed results. In particular, in section 2.7, it is provided a clear explanation of the concepts of structured singular value $(\mu)$ and DK-iteration, and presented Algorithm 1, which indicates the Matlab ${ }^{\circledR}$ functions that can be used. In Chapter 3, results for SISO case 
are presented. In Chapter 4, one presents the main theoretical contributions of the work, all for MIMO systems with and without delay. In Chapter 5, the modeling of Alzheimerlike diseases via Hopfield networks are presented, and then the application of the Theorem 25, which solves the problem of memory failure in the modeled disease. Finally, in Chapter 6 , the conclusions, comments and a prospect for future research are presented. 


\section{THEORETICAL BACKGROUND}

This chapter presents the theoretical basis for the results which will be presented in the following chapters.

\subsection{Basic Definitions}

This section gives some basic definitions about norms, stability in the sense of Lyapunov, absolute stability, and robust stability.

\subsubsection{Norms}

Norms are functions that measure the size of a vector, matrix, signal or system. In this subsection, with content based on (SKOGESTAD; POSTLETHWAITE, 2007), the concept of norm will be presented, and more specifically, the definitions of norms of vectors and matrices are given.

Definition 1. A norm in a vector space $V$ is a real number, denoted $\|v\|$, that satisfies the following properties.

1. $\|v\| \geq 0$ with $\|v\|=0$ if, and only if, $v=0$.

2. $\|\alpha v\|=|\alpha|\|v\|$.

3. $\|u+v\| \leq\|u\|+\|v\|$.

More precisely, $v$ is an element in a vector space $V$ over the field $\mathbb{C}$ of complex numbers, and the properties above must be satisfied $\forall v, u \in V$ and $\forall \alpha \in \mathbb{C}$.

\subsubsection{Vector Norm}

Examples of norms for $v=\left[v_{1}, v_{2}, \ldots, v_{n}\right]^{T}$ include: 
- Vector 1-norm.

$$
\|v\|_{1}=\sum_{i=1}^{n}\left|v_{i}\right|=\left|v_{1}\right|+\left|v_{2}\right|+\ldots+\left|v_{n}\right|
$$

- Vector 2-norm (Euclidean norm).

$$
\|v\|_{2}=\sqrt{\sum_{i=1}^{n}\left|v_{i}\right|^{2}}=\sqrt{\left|v_{1}\right|^{2}+\left|v_{2}\right|^{2}+\ldots+\left|v_{n}\right|^{2}}
$$

- Vector $\infty$-norm.

$$
\|v\|_{\infty}=\max _{i=1: n}\left|v_{i}\right|=\max \left\{\left|v_{1}\right|,\left|v_{2}\right|, \ldots,\left|v_{n}\right|\right\}
$$

\subsubsection{Matrix Norm}

A matrix norm can be defined for any matrix vector space. In this thesis, norms will be defined for real and complex constant matrices and for variable matrices, like for example the matrix of transfer function $G(j \omega)$, which will result in a function of $\omega$.

Definition 2. A norm on a matrix $\|A\|$ is a matrix norm if, in addition to properties of the Definition 1, it also satisfies the multiplicative property (also called consistency condition):

$$
\|A B\| \leq\|A\| \cdot\|B\|
$$

The three main matrix norms are:

- Sum matrix norm.

$$
\|A\|_{s u m}=\sum_{i j}\left|a_{i j}\right|
$$

- Frobenius matrix norm (or Euclidean norm).

$$
\|A\|_{F}=\sqrt{\sum_{i j}\left|a_{i} j\right|^{2}} .
$$

- Max element norm (or $\infty$-norm).

$$
\|A\|_{\infty}=\max _{i}\left(\sum_{j}\left|a_{i j}\right|\right) .
$$




\subsubsection{Stability Definitions}

In this section, some stability concepts and definitions are presented. First of all, the stability in the sense of Lyapunov, which is the basic one, is presented. After that, absolute stability, which is necessary for this thesis, is presented, as well as the definition of robust stability, which connects to the concept of absolute stability. Those definitions are presented in (GUZMAN, 1980), (LIAO; YU, 2008), (OGATA, 2010) and (SKOGESTAD; POSTLETHWAITE, 2007) in greater detail.

\subsubsection{Stability in the Sense of Lyapunov}

Stability in the sense of Lyapunov is largely used in dynamical systems and is the basis for defining absolute stability. Suppose a general autonomous system, which is a time-independent set of ordinary differential equations in an initial value problem:

$$
\left\{\begin{array}{llc}
\dot{x}(t) & = & f(x(t)), \\
x\left(t_{0}\right) & = & x_{0}
\end{array}\right.
$$

where $f: D \rightarrow \mathbb{R}^{n}$, for $D \subset \mathbb{R}^{n}$ is an open set, satisfies conditions that guarantee existence and uniqueness of solutions, and suppose that a solution is denoted by $x\left(t ; t_{0}, x_{0}\right)$ and can be extended to $\left[t_{0},+\infty\right)$. If there is a point $x^{*}$ such that that $f\left(x^{*}\right)=0$, then $x\left(t, t_{0}, x^{*}\right) \equiv 0$ is a trivial solution, also called the null solution or equilibrium point.

Definition 3. The equilibrium point $x^{*}$ of (2.8) is said to be stable in sense of Lyapunov if for all $\epsilon>0$ there is $\delta>0$ ( $\delta$ is dependent on $\epsilon$ ) such that for all $x_{0} \in D$ with $\left\|x_{0}-x^{*}\right\|<\delta$ one verifies

$$
\left\|x\left(t ; t_{0}, x_{0}\right)-x^{*}\right\| \leq \epsilon, \forall t \in\left[t_{0},+\infty\right)
$$

where $\|\cdot\|$ is a norm in $\mathbb{R}^{n}$.

This means that if there exists a ball of radius $\delta$ centered at $x^{*}$ such that for any trajectory $x\left(t ; t_{0}, x_{0}\right)$ with initial condition $x_{0}$ belonging to that ball, then $x\left(t ; t_{0}, x_{0}\right)$ remains inside another ball of radius $\epsilon$ centered at $x^{*}$ as time passes. Figure 7 illustrates this idea.

Definition 4. The equilibrium point $x^{*}$ of (2.8) is said to be locally asymptotically stable if it is stable in the sense of Lyapunov and there is $\eta>0$ such that if $\left\|x_{0}-x^{*}\right\| \leq \eta$ for all $x_{0} \in D$, then

$$
\lim _{t \rightarrow+\infty}\left\|x\left(t ; t_{0}, x_{0}\right)\right\|=x^{*}
$$


Figure 7: Geometric notion of stability in dimension 2.

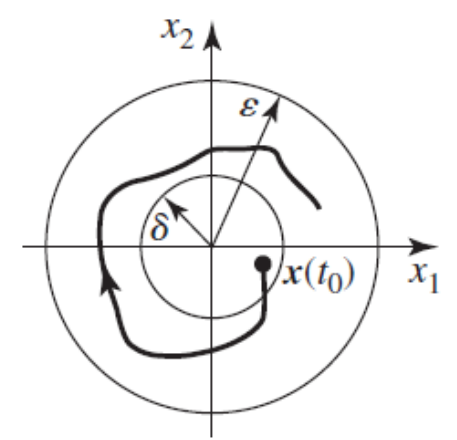

Source: (ZAK, 2003).

This definition says that $x^{*}$ will be an asymptotically stable equilibrium point if all trajectories $x\left(t ; t_{0}, x_{0}\right)$, whose initial conditions are contained in a ball of radius $\eta$ with center at $x^{*}$, approach $x^{*}$ as time passes. If this ball has finite radius, then $x^{*}$ is locally asymptotically stable, and if this ball has infinite radius, then the equilibrium point is globally asymptotically stable. It also means that the equilibrium point must be unique.

Figure 8: Geometric notion of asymptotic stability in dimension 2.

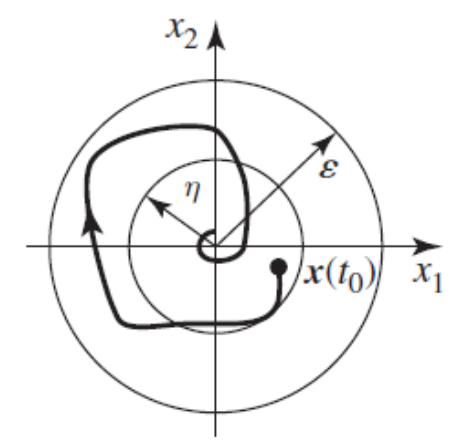

Source: (ZAK, 2003).

Definition 5. The equilibrium point $x^{*}$ of (2.8) is said to be globally asymptotically stable if it is stable in the sense of Lyapunov and for all $x_{0} \in \mathbb{R}^{n}$ one has:

$$
\lim _{t \rightarrow+\infty}\left\|x\left(t ; t_{0}, x_{0}\right)\right\|=x^{*}
$$

Hence an equilibrium point that is not stable is called unstable, that is, if there exists $x_{0}$ such that, independent of how close it is to $x^{*}$, the corresponding trajectory moves away from the neighborhood of radius $\epsilon$ in a finite time $t$, then the point is unstable.

A linear system like in Figure 10, which is very common in control applications and appears in the LP, has the following equation: 
Figure 9: Geometric notion of instability in dimension 2.

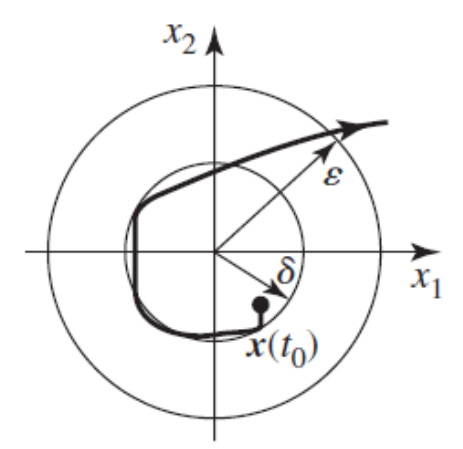

Source: (ZAK, 2003).

$$
\left\{\begin{array}{l}
\dot{x}=A x+B r_{1}, \\
\sigma=C x+D r_{1},
\end{array}\right.
$$

where $x \in \mathbb{R}^{n}$ is the state vector, $r_{1} \in \mathbb{R}^{m}$ is the input vector, $\sigma \in \mathbb{R}^{m}$ is the output vector, and the matrices $A \in \mathbb{R}^{n \times n}, B \in \mathbb{R}^{n \times m}$ and $C \in \mathbb{R}^{m \times n}$ are known and fixed. The transfer function matrix, that relates outputs and inputs, is given by:

$$
G(s)=C(s I-A)^{-1} B+D .
$$

Figure 10: Block diagram of linear system.

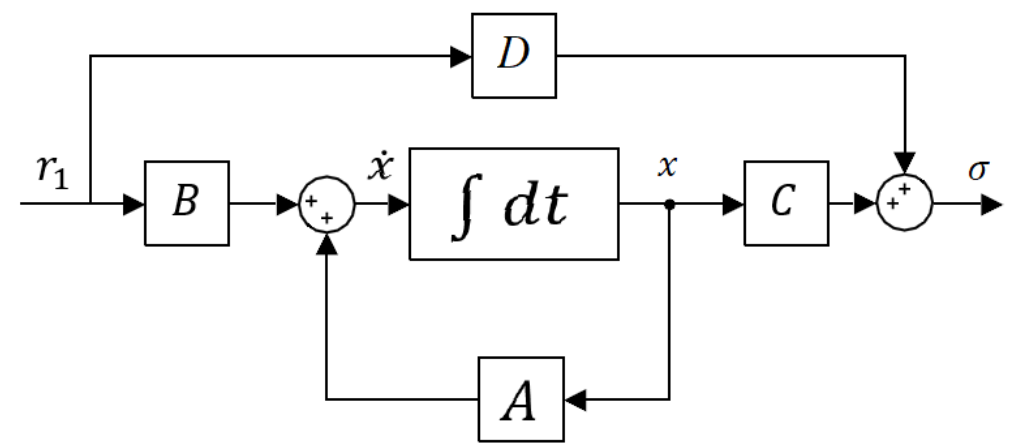

Source: Adapted of (OGATA, 2010).

In the following, stability is defined for a linear and time-invariant control system (OGATA, 2010).

Definition 6. A linear and time-invariant control system of equations (2.12) or (2.13) is stable if the output always returns to the equilibrium state when the system is subjected to an initial condition.

Remark 1. Note that the Definition 6 is derived from the Definition 5. Where in the Definition 6 is considered an output $\sigma(t)$ that is composed of the solution $x\left(t ; t_{0}, x_{0}\right)$. For LP systems like (1.1) and (1.2), one has $D=0$. 
Definition 7. It is said that a real or complex matrix $n \times n$ is Hurwitz if all its eigenvalues have negative real part.

Remark 2. For the systems (2.12) or (2.13) to be stable, the poles of $G(s)$ must be in the open complex left half-plane, that is, they must have negative real part. In equivalence to this statement it is sufficient and necessary that A be Hurwitz (SKOGESTAD; POSTLETHWAITE, 2007).

\subsubsection{Absolute Stability}

Absolute stability is a special type of stability that is related to Lurie type system as presented by equations $(1.1),(1.2),(1.3)$ or (1.4). One can see that it refers to a closedloop nonlinear system. Below it is presented the definition of absolute stability for the MIMO case. For the SISO case, just consider $j=1$.

Definition 8. (Absolute Stability) Assuming null input $\left(r_{1}=0\right)$, it is said that the system (1.2) is absolutely stable if the zero solution (equilibrium point) of (1.2) is globally asymptotically stable in Lyapunov sense for $f_{j}\left(\sigma_{j}\right) \in F_{\left(0, k_{j}\right]}$, that is, $\forall \epsilon>0, \exists \delta(\epsilon)>0$, such that the solution $x(t):=x\left(t, t_{0}, x_{0}\right)$ of the initial value problem associated to (1.2) is unique and satisfies:

$$
\|x(t)\|=\sum_{j=1}^{m}|x(t)|<\epsilon, \quad \forall t \geq t_{0}, \quad \text { if } \quad\left\|x_{0}\right\|<\delta(\epsilon),
$$

and for any $x_{0} \in \mathbb{R}^{n}$ :

$$
\lim _{t \rightarrow \infty}\|x(t)\|=0
$$

Remark 3. Note that the definition of absolute stability indicates an unique equilibrium point. In fact, it is a necessary condition that the equilibrium point be unique for the system to be globally asymptotically stable.

Remark 4. Considering the system (1.2) and the Definition 8, it is presented the formulation for the Lurie problem in MIMO case for $f_{j}\left(\sigma_{j}\right) \in F_{\left(0, k_{j}\right]}$. Similar definitions may also be formulated for the families $F_{\left(0, k_{j}\right)}, F_{\left[\underline{k}_{j}, \bar{k}_{j}\right]}$, and $F_{\infty}$.

\subsubsection{Robust Stability}

Throughout the history of the study of Lurie's problem, the conjecture or problem of Aizerman (AIZERMAN, 1947) initially distinguished itself, where an Aizerman type system is a particular case of a Lurie type system (PINHEIRO, 2015). In his conjecture, 
Aizerman said: the Hurwitz and absolute stability sectors coincide (RASVAN; POPESCU, 2012). It was proved that the conjecture was valid for systems of dimension 1 and dimension 2 (KRASOVSKII, 1953). However, Pliss (PLISS, 1958) presented a counterexample in dimension 3 invalidating the conjecture. Therefore, it is important to emphasize that in this work one is not working on Aizerman's conjecture, but on Lurie's problem, that is, absolute stability analysis.

When one talks about absolute stability, by definition it is being considered that the nonlinearity belongs to a class of functions mapped in a certain sector. This indicates one can convert to a robustness problem, where the following question is valid: If one has a nonlinearity in feedback, for the class of nonlinearities defined as a sector, where does stability exist? This can be understood as the search for global asymptotic stability conditions, which are like tests that check the parameters of the system and the sectors that map the nonlinearities where in fact the system may have stability. Thus, providing sufficient conditions that are valid for certain classes of functions, but which usually has some conservatism.

The nonlinearity of the Lurie type system in SISO case of the equation (1.1) is mapped according to Figure 11 and changed in equation (2.27), and for MIMO case of the equation (1.2) is mapped according to Figure 16 and changed in equation (2.28). Thus, the problem of absolute stability of the Lurie type systems can be studied as a problem of analysis of robust stability. In order to use this idea, the definition of robust stability, in SISO case, is formulated:

Definition 9. Assuming null input $\left(r_{1}=0\right)$, it is said that the system in (2.27) is robustly stable if for any perturbation $\alpha$, the output $\sigma_{\alpha}$ always returns to the equilibrium state (zero), i.e., $\lim _{t \rightarrow \infty} \sigma_{\alpha}=0$.

For the MIMO case follows the definition:

Definition 10. Assuming null input $\left(r_{1}=0\right)$, one says that the uncertain but fixed system (2.28) is robustly stable if for any value of $\alpha_{j}$ in a certain interval, the system remains stable (i.e. asymptotically stable).

More details about the connection between absolute stability and robust stability will be given in section 2.4 and Lemma 2 . 
Figure 11: Mapping $\quad 0<f(\sigma) \leq k \sigma$.

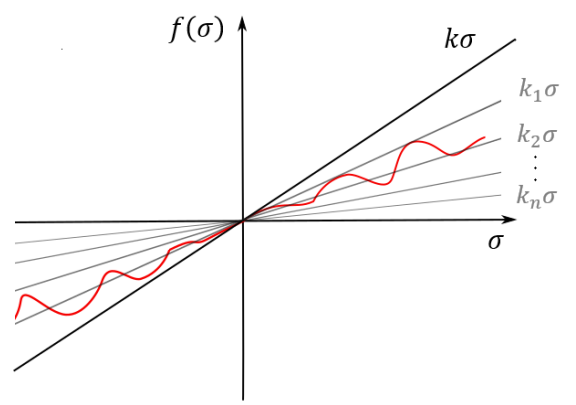

Source: Author.

\subsection{Three Theorems for Absolute Stability}

Three basic theorems that provide sufficient conditions for absolute stability are presented in this section. The first is Popov's criterion (POPOV, 1961) for SISO system. The second is a theorem, due to Liao (LIAO, 1993), that establishes a condition from the coefficients of a MIMO Lurie type system in state space, which in this work is called explicit condition. And the last theorem, is the generalized Popov's criterion, due to Haddad (HADDAD; BERNSTEIN, 1993), which brings condition from the frequency response for MIMO Lurie system. These theorems will be used to compare with the results of this thesis.

\subsubsection{Popov Criterion}

The next theorem is well-established result in the literature that will be used to study and compare a result presented in this work. The Popov's criterion (POPOV, 1961), one of the most used to verify the stability of Lurie type system in SISO case. In Figure 12 it can be observed the graphical representation of Popov's criterion according to Theorem 1. This theorem establishes only sufficient condition.

Theorem 1. Let $G_{1}$ and $G_{2}$ be real transfer functions and let $P$ be complex function, such that, $G(j w)=G_{1}(w)+j G_{2}(w)$ and $P(j w)=G_{1}(w)+j w G_{2}(w)$. If the system (1.1) satisfies the conditions:

i. A is Hurwitz and the pair $(A, b)$ is controllable.

ii. The nonlinearity $f$ belongs to the sector $(0, k]$.

iii. $\exists q>0, q \in R$, such that, $x-q y+\frac{1}{k}>P(j w), \forall w \geq 0$, where in complex plane $x \in R$ and $y \in R$ represent real and imaginary axis, respectively. 
Then, the zero solution (equilibrium point) of the system (1.1) is absolutely stable.

Proof. It can be found in (KHALIL, 2002), and in (SLOTINE; LI, 1991) one has the graphical interpretation.

Figure 12: The Popov's criterion - Theorem 1.

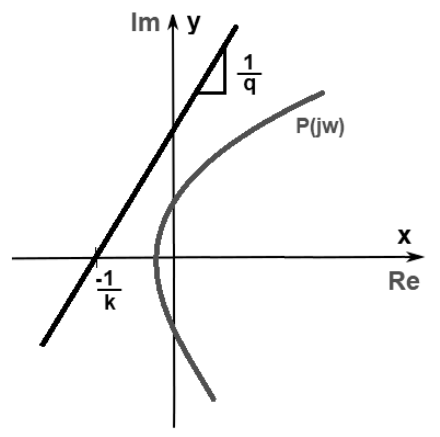

Source: Author.

\subsubsection{Theorem of Explicit Condition}

The next theorem, due to Liao (LIAO, 1993), establishes a sufficient condition for the MIMO case. This theorem provides explicit conditions. This theorem also will be used to compare with one of the results of this thesis.

Theorem 2. The zero solution of (1.4) is absolutely stable if one of the sets of conditions is satisfied:

1) $\tilde{a}_{i i}<0$ for $i=1, \ldots, n$, and the matrix $\left((-1)^{\bar{\delta}_{i j}}\left|\tilde{a}_{i j}\right|\right)_{n_{x} n}$ is Hurwitz $\left(\bar{\delta}_{i j}\right.$ is the Kronecker delta).

2) There is a constant $k_{l}>0$ for $l=1, \ldots, n$ such that:

$$
\left\{\begin{array}{c}
k_{l} \tilde{b}_{l l} \leq \tilde{a}_{l l}, \quad k_{l}\left|\tilde{b}_{i l}\right|<\left|\tilde{a}_{i l}\right|, \quad \text { for } \quad l=1, \ldots, n, \quad i=1, \ldots, n, \quad i \neq l, \\
\text { or } \\
k_{l} \tilde{b}_{l l}<\tilde{a}_{l l}, \quad k_{l}\left|\tilde{b}_{i l}\right| \leq\left|\tilde{a}_{i l}\right|, \quad \text { for } \quad l=1, \ldots, n, \quad i=1, \ldots, n, \quad i \neq l,
\end{array}\right.
$$

or

$\left.1^{\prime}\right) \tilde{a}_{i i}<0$ for $i=1, \ldots, n$, and the matrix $\left((-1)^{\bar{\delta}_{i j}}\left|\alpha_{i j}\right|\right)_{n_{x} n}$ is Hurwitz.

2') There is a constant $k_{l}>0$ for $l=1, \ldots, n$ such that: 


$$
\left\{\begin{array}{c}
k_{l} \tilde{a}_{l l} \leq \tilde{b}_{l l}, \quad k_{l}\left|\tilde{a}_{i l}\right|<\left|\tilde{b}_{i l}\right|, \quad \text { for } \quad l=1, \ldots, n, \quad i=1, \ldots, n, \quad i \neq l \\
\text { or } \\
k_{l} \tilde{a}_{l l}<\tilde{b}_{l l}, \quad k_{l}\left|\tilde{a}_{i l}\right| \leq\left|\tilde{b}_{i l}\right|, \quad \text { for } \quad l=1, \ldots, n, \quad i=1, \ldots, n, \quad i \neq l
\end{array}\right.
$$

Proof. It can be found in (LIAO, 1993).

\subsubsection{Generalized Popov's Criterion}

The next theorem is know as generalized Popov's criterion (HADDAD; BERNSTEIN, 1993). This theorem presents a sufficient condition for MIMO Lurie type systems with $\tilde{C}$ equal to the identity matrix. It will be used to compare the conservatism of the results of this work

Theorem 3. Let $\left[0, k_{j}\right]$ be the sectors that limit $\tilde{f}_{j}\left(\tilde{\sigma}_{j}\right)$ such that $\tilde{f}_{j} \in F_{\left(0, k_{j}\right]}$ and $K=$ $\operatorname{diag}\left[k_{j}\right]$ for $j=1, \ldots, m$. If $(\tilde{A}, \tilde{B})$ is controllable, $(\tilde{A}, \tilde{C})$ is observable and there exists $N=\operatorname{diag}\left[n_{j}\right]$, where $n_{j}$ are nonnegative constants such that:

$$
Q(s)=K^{-1}+(I+N s) \tilde{G}(s)>0,
$$

(i.e., $Q$ is strongly positive real), where $\tilde{G}(s)=\tilde{C}(s I-\tilde{A})^{-1} \tilde{B}$, then the zero solution of (1.4) is absolutely stable.

Proof. It can be found in (HADDAD; BERNSTEIN, 1993).

Remark 5. According to (HADDAD; BERNSTEIN, 1993), Q(s) is strongly positive real if: i) $Q(s)$ is asymptotically stable; ii) $H(j \omega)=Q(j \omega)-Q^{\prime}(j \omega)>0$ for all real $\omega$; and iii) $H(\infty)=Q(\infty)-Q^{T}(\infty)>0$.

\subsection{Linear Fractional Transformations}

The Linear Fractional Transformation (LFT) is well known in the theory of complex variables for scalars (ZHOU; DOYLE; GLOVER, 1995), and its generalization for matrices was first used in the control literature by Doyle (DOYLE, 1984), who realized that a feedback system can be represented in this way. In fact, there are two forms of LFT: lower and upper (see Figure 13). The lower LFT is typically used when one is designing and analysing feedback controllers (that is $K$ ), and the upper LFT is typically used when one wants to isolate the uncertainties in a single subsystem, that is $\Delta$ (or vice-versa). In this work, it is used the lower LFT for representing uncertainties. 
Figure 13: (a) $S$ as lower LFT in terms of $K$.

(b) $S$ as upper LFT in terms of $\Delta$.
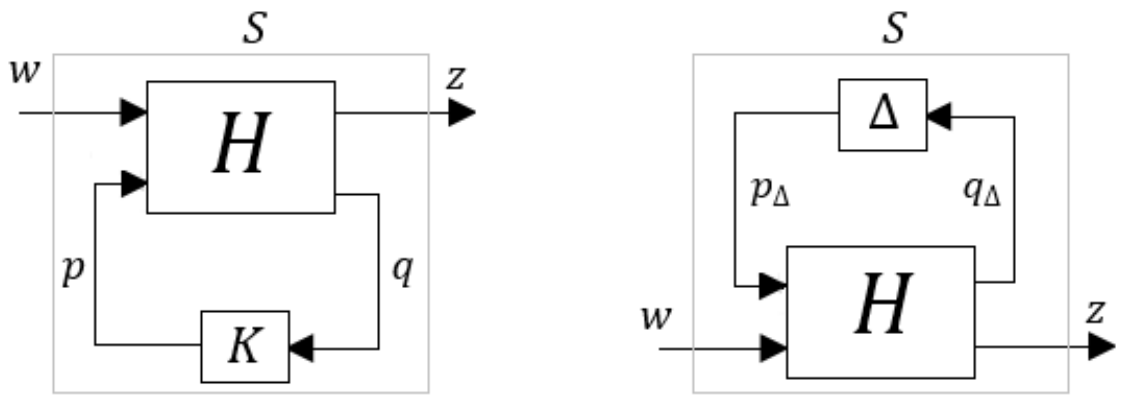

Source: Author.

Given a matrix of transfer function $H$ of dimensions $\left(n_{1}+n_{2}\right) \times\left(m_{1}+m_{2}\right)$ and partitioned as follows:

$$
H=\left[\begin{array}{ll}
H_{11} & H_{12} \\
H_{21} & H_{22}
\end{array}\right],
$$

and the matrices $\Delta \in \mathbb{R}^{m_{1} \times n_{1}}$ and $K \in \mathbb{C}^{m_{2} \times n_{2}}$ with compatible dimension, the formulas for lower and upper LFT are:

$$
\begin{aligned}
& F_{L}(H, K) \triangleq H_{11}+H_{12} K\left(I-H_{22} K\right)^{-1} H_{21} \\
& F_{U}(H, \Delta) \triangleq H_{22}+H_{21} \Delta\left(I-H_{11} \Delta\right)^{-1} H_{12}
\end{aligned}
$$

where subscript $L$ denotes lower and subscript $U$ denotes upper. The matrices of transfer function $F_{L}(H, K)$ and $F_{U}(H, \Delta)$ are the closed-loop matrices of transfer functions of the system. Formulas (2.17) and (2.18) can be easily obtained using block diagram algebra.

In applications, the control designer must obtain, first of all, the system model, normalize the uncertain parameters and isolate them in a matrix $\Delta$, which is block diagonal with real or complex entries $\delta_{i j}$ such that $\left|\delta_{i j}\right| \leq 1$. The matrix $H$ is then called the nominal plant. If the model is in the form of block diagrams (in which each block is a transfer function), there is no systematic method to obtain that description (see (SKOGESTAD; POSTLETHWAITE, 2007) for some examples). If, on the other hand, the model is in state-space form, the process is much simpler (the uncertain matrices must be written as linear combinations of fixed matrices with uncertain coefficients $\delta$ ). In fact, in the proofs of the developed results presented here, it is assumed that the model is in the state-space form. 


\subsubsection{Linear State-Space System with Normalized Uncertainties}

Next, a methodology proposed by (MORTON; MCAFOOS, 1985) is presented, which will be used to prove the Lemma 4. Consider an uncertain state-space model, where $A_{0}, B_{0}, C_{0}$ and $D_{0}$ are the nominal matrices. If $\alpha_{j}$ is parameterized using a normalized uncertainty $\delta_{j}$ such that $\left|\delta_{j}\right| \leq 1$, for $j=1, \ldots, m$, then:

$$
\begin{gathered}
{\left[\begin{array}{c}
\dot{x} \\
\sigma
\end{array}\right]=\left[\begin{array}{cc}
A_{0}+\sum_{j=1}^{m} \delta_{j} A_{j} & B_{0}+\sum_{j=1}^{m} \delta_{j} B_{j} \\
C_{0}+\sum_{j=1}^{m} \delta_{j} C_{j} & D_{0}+\sum_{j=1}^{m} \delta_{j} D_{j}
\end{array}\right]\left[\begin{array}{l}
x \\
r_{1}
\end{array}\right]} \\
=\left(\left[\begin{array}{cc}
A_{0} & B_{0} \\
C_{0} & D_{0}
\end{array}\right]+\sum_{j=1}^{m} \delta_{j}\left[\begin{array}{cc}
A_{j} & B_{j} \\
C_{j} & D_{j}
\end{array}\right]\right)\left[\begin{array}{l}
x \\
r_{1}
\end{array}\right],
\end{gathered}
$$

where for each $\mathrm{j}=1, \ldots, \mathrm{m}$ :

$$
\left[\begin{array}{cc}
A_{j} & B_{j} \\
C_{j} & D_{j}
\end{array}\right] \in \mathbb{R}^{(n+m) \times(n+m)}
$$

Let

$$
\rho_{j}=\operatorname{rank}\left[\begin{array}{cc}
A_{j} & B_{j} \\
C_{j} & D_{j}
\end{array}\right]
$$

and factor each matrix as:

$$
\left[\begin{array}{ll}
A_{j} & B_{j} \\
C_{j} & D_{j}
\end{array}\right]=\left[\begin{array}{c}
E_{j} \\
F_{j}
\end{array}\right]\left[\begin{array}{ll}
G_{j} & H_{j}
\end{array}\right]
$$

where

$$
\left[\begin{array}{c}
E_{j} \\
F_{j}
\end{array}\right] \in \mathbb{R}^{(n+m) \times \rho_{j}} \quad, \quad\left[\begin{array}{ll}
G_{j} & H_{j}
\end{array}\right] \in \mathbb{R}^{\rho_{j} \times(n+m)} .
$$

Now, define a linear system $\Lambda$, with extra inputs and outputs via the state equations:

$$
\left[\begin{array}{l}
\dot{x} \\
\sigma \\
q_{1} \\
\vdots \\
q_{m}
\end{array}\right]=\underbrace{\left[\begin{array}{ccccc}
A_{0} & B_{0} & E_{1} & \ldots & E_{m} \\
C_{0} & D_{0} & F_{1} & \ldots & F_{m} \\
G_{1} & H_{1} & 0 & \ldots & 0 \\
\vdots & \vdots & \vdots & \ddots & \vdots \\
G_{m} & H_{m} & 0 & \ldots & 0
\end{array}\right]}_{\Lambda}\left[\begin{array}{c}
x \\
r_{1} \\
p_{1} \\
\vdots \\
p_{m}
\end{array}\right],
$$

as shown in Figure 14. The uncertain system is represented as a LFT around $\Lambda$, namely $\sigma=F_{L}(\Lambda, \Delta) r_{1}$, where $\Delta$ maps $q \rightarrow p$ (see Figure 15), and has the following structure:

$$
\Delta=\left\{\operatorname{diag}\left[\delta_{1} I_{\rho_{1}}, \ldots, \delta_{m} I_{\rho_{m}}\right]: \delta_{j} \in \mathbb{R}\right\}
$$


Figure 14: $\Lambda$ System.

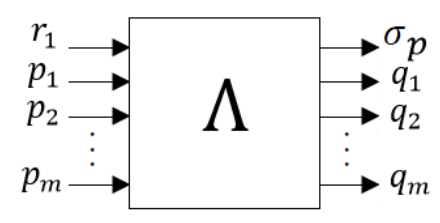

Source: Author.
Figure 15: $\Lambda-\Delta$-Structure.

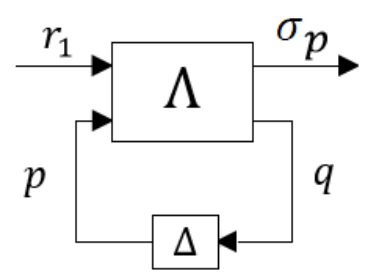

Source: Author.

\subsection{Modeling Nonlinearity by Means of Parametric Uncertainties and LFT}

In this section, one presents the method of modeling nonlinearities as is done in the Lurie problem. In subsections 2.4.1 and 2.4.2, the strategies adopted in this work for modeling nonlinearity via parametric uncertainties for use in robust control theory are presented for the SISO and MIMO cases of Lurie type systems. Finally, in the subsection 2.4.3 a summary is presented in a few steps, of how to obtain a linearized Lurie type system via parametric uncertainties with LFT.

\subsubsection{SISO Case}

According to the approach presented in this work, nonlinearity has to be modelled as a parametric uncertainty. The following is a methodology used by (PINHEIRO; COLÓN, 2017). The nonlinearity $f$ of the Figure 4 must be replaced by a parametric uncertainty. The resulting system will be an equivalent family of plants $G_{\alpha}$. The idea is to replace $f$ by a set of linear functions $k \sigma$ (see Figure 11), where $k$ is the uncertain parameter.

In fact, any nonlinearity is being mapped according to this constraint in the first and third quadrants. This makes sense for Lurie problem, because the function $f(\sigma)$ is not known, except that it is between the first and third quadrants. For more information on linear spaces and mapping see (DULLERUD; PAGANINI, 2013). In practice, the block $f(\sigma)$ can be replaced by the uncertain block $\alpha$, where $0<\alpha \leq k$.

From the diagram of Figure 4 (with $\alpha$ in place of $f$ ), the perturbed transfer function $G_{\alpha}(s)$ is obtained as follows:

$$
\begin{gathered}
\dot{x}=A x+b\left(r_{1}-\alpha c^{T} x\right) \quad \rightarrow \quad \dot{x}=A x+b r_{1}-b \alpha c^{T} x \\
\rightarrow \quad \dot{x}=\left(A-b \alpha c^{T}\right) x+b r_{1} .
\end{gathered}
$$


Making $A_{\alpha}=\left(A-b \alpha c^{T}\right)$, one has:

$$
\left\{\begin{array}{l}
\dot{x}=A_{\alpha} x+b r_{1} \\
\sigma_{\alpha}=c^{T} x .
\end{array}\right.
$$

Therefore, the perturbed transfer function of the plant is given by:

$$
G_{\alpha}(s)=c^{T}\left(s I-A_{\alpha}\right)^{-1} b
$$

or, making $G(s)=c^{T}(s I-A)^{-1} b$ in linear part of Figure 4, one has:

$$
G_{\alpha}(s)=\frac{G(s)}{1+\alpha G(s)} .
$$

The following lemma is an important result adapted from (GUZMAN, 1980) and (LIAO; YU, 2008), that makes possible the study of the absolute stability of the system (1.1) by means of the study of the robust stability of system (2.27).

Lemma 1. For some class of functions in a sector, if the linear fixed and uncertain system (2.25), (2.26) or (2.27) are robustly stable, then the system (1.1) is absolutely stable.

Proof. The proof is analogous to presented in Lemma 2.

\subsubsection{MIMO Case}

Above it is presented a methodology used by (PINHEIRO; COLÓN, 2017) for the SISO case. Here one extends it to the MIMO case. The goal is to replace the unknown but fixed static nonlinearity $f$ of $(1.2)$ by a set of uncertain but fixed linear functions $\alpha \sigma(x(t))$, where the parameters $\alpha=\left[\alpha_{1}, \ldots, \alpha_{m}\right]$ are fixed but unknown real constants (see Figure 16), such that $0<\alpha_{j} \leq k_{j}$. The resulting system is an equivalent family of plants $G_{p}(s)$, as is common in robust control. In fact, any nonlinearity is mapped according to this constraint in the first and third quadrants (DULLERUD; PAGANINI, 2013). Thus, the closed-loop linearized model of the system (1.2) is as follows:

$$
\begin{gathered}
\dot{x}=A x+B\left(r_{1}-\alpha C x\right) \rightarrow \dot{x}=A x+B r_{1}-B \alpha C x \\
\rightarrow \quad \dot{x}=(A-B \alpha C) x+B r_{1} .
\end{gathered}
$$

Making $A_{\alpha}=(A-B \alpha C)$ (see (4.1)), one has: 


$$
\left\{\begin{array}{l}
\dot{x}=A_{\alpha} x+B r_{1} \\
\sigma=C x
\end{array}\right.
$$

Figure 16: Mapping $\quad 0<f_{j}\left(\sigma_{j}\right) \leq k_{j} \sigma_{j}$.
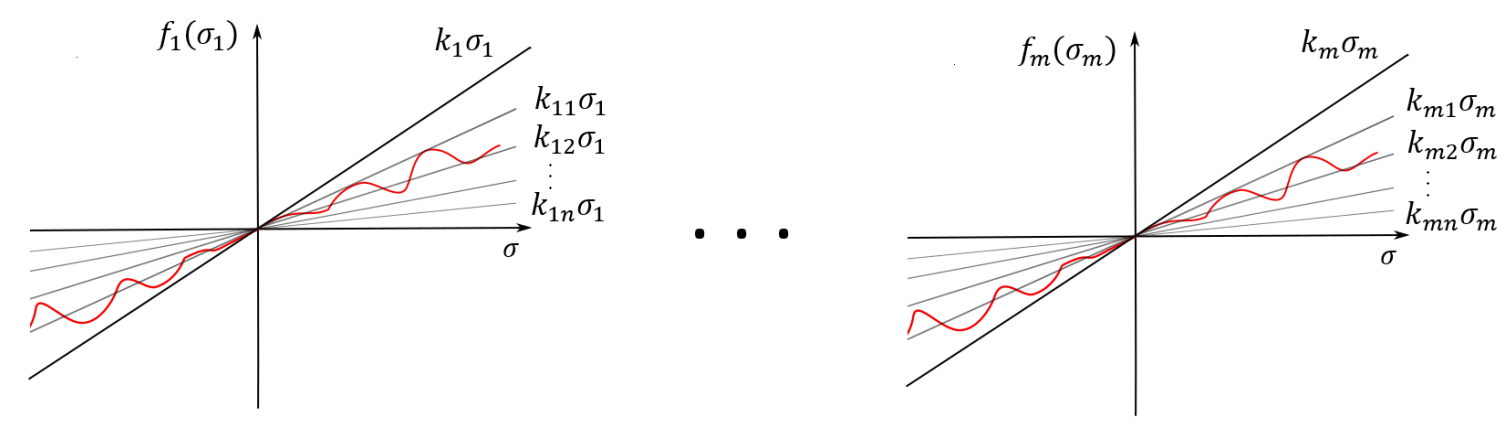

Source: Author.

It is studied the following lemma which is an important result adapted from (GUZMAN, 1980) and (LIAO; YU, 2008), that makes possible the study of the absolute stability of the system (1.2) by means of the study of the robust stability of system (2.28). It is given just a sketch of the proof here, but the interested readers can find the complete proof in (GUZMAN, 1980) pp. 190, Theorem 6.4.3; and in (LIAO; YU, 2008) pp. 158, Theorem 7.4, from sufficiency onward.

Lemma 2. For some class of functions in a sector, if the linear fixed and uncertain system (2.28) is robustly stable, then the system (1.2) is absolutely stable.

Sketch of Proof. Adding $(B \alpha \sigma-B \alpha \sigma)$ in (1.2), the equation can be rewritten as, $\dot{x}=A x+B \alpha \sigma-B \alpha \sigma-B f(\sigma)$ and replacing $\sigma$ by $C x$, one has: $\dot{x}=(A-B \alpha C) x+B \alpha C x-$ $B f(C x)$. By applying Lagrange's formula, the solution $x\left(t, t_{0}, x_{0}\right)$ of (1.2) satisfies:

$$
x(t)=e^{A_{\alpha}\left(t-t_{0}\right)} x_{0}+\int_{t_{0}}^{t} e^{A_{\alpha}(t-\tau)} \underbrace{B[\alpha C x(\tau)-f(C x(\tau))]}_{F(\tau, x)} d \tau .
$$

Now, if the uncertain but fixed system (2.28) is robustly stable, then $A_{\alpha}$ is Hurwitz stable (all eigenvalues with real part negative) for all $\alpha$ in a certain interval, which means that there exist constants $L \geq 1$ and $\beta>0$ such that:

$$
\left\|e^{A_{\alpha}\left(t-t_{0}\right)}\right\| \leq L e^{-\beta\left(t-t_{0}\right)}, \quad t \geq t_{0}
$$

which means that the part of the solution that depends on the initial condition goes to zero asymptotically. In order to have the integral part going to zero, some additional 
conditions in $F(\tau, x)$ are necessary. Let us suppose that the second condition of theorem 4.3 of (LIAO; YU, 2008) is satisfied. Then:

1) $\forall \epsilon>0, \exists \delta(\epsilon)>0$ and $t_{1} \geq t_{0}$, such that $\left\|x_{0}\right\|<\delta(\epsilon)$ implies $\|x(t)\|=\| e^{A_{\alpha}\left(t-t_{0}\right)} x_{0}+$ $\int_{t_{0}}^{t} e^{A_{\alpha}(t-\tau)} B[\alpha C x(\tau)-f(C x(\tau))] d \tau \|<\epsilon$; and

2) for any $x_{0} \in R$, one has:

$$
\begin{aligned}
& 0 \leq \lim _{t \rightarrow \infty}\|x(t)\| \leq \lim _{t \rightarrow \infty} L e^{-\beta\left(t-t_{0}\right)} \\
& \quad+\lim _{t \rightarrow \infty} \int_{t_{0}}^{t} L e^{-\beta\left(t-t_{0}\right)} B[\alpha C x(\tau)-f(C x(\tau))] d \tau=0 .
\end{aligned}
$$

Then, for the class of functions in a sector that satisfy the Liao's condition, robustness of stability implies absolute stability. Thus, the results presented in this thesis are effective when the problems under analysis coincide with this class of functions, where sufficient conditions of absolute stability are obtained.

\subsubsection{Lurie type System via Parametric Unicertainty and LFT}

In order to model the nonlinear system by a linear normalized uncertain system with LFT, one must follow the steps:

1. Replace the nonlinear functions $f(\sigma)$ with the uncertain linear functions $\alpha \sigma$, where $\alpha$ is the vector of uncertain parameters such as $\alpha=\left[\alpha_{1}, \ldots, \alpha_{m}\right]$.

2. Normalize the uncertainties $\alpha_{j}$, obtaining $\left|\delta_{j}\right| \leq 1$, and putting the system if the form of (2.19).

3. Find the ranks of the matrices like in (2.21).

4. Calculate the matrices $E_{j}, F_{j}, G_{j}$ and $H_{j}$, like in (2.22).

5. Find the matrices $\Lambda$ and $\Delta$ like in (2.23) and (2.24), respectively.

\subsection{Mixed-Sensitivity Method}

In this section, the robust control theory is presented, in particular the $\mathcal{H}_{\infty}$ mixedsensitivity $(\mathrm{S} / \mathrm{KS} / \mathrm{T})$ method, necessary for the results of this work. In order to design a 
robust controller, a $G_{p}(s)$ is used, which represents the perturbed transfer function of a plant family including the normalized $\delta$-uncertainty, according to $(2.32)$ and Figure 17:

Figure 17: Perturbed transfer function.

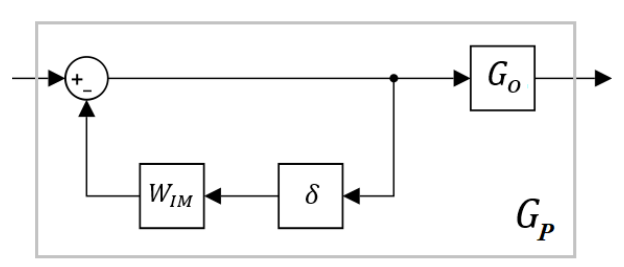

Source: Author.

$$
G_{p}(s)=\frac{G_{o}(s)}{1+W_{I M}(s) \delta}, \quad|\delta| \leq 1 .
$$

The transfer function $G_{o}(s)$ is the new nominal model of the plant and $W_{I M}$ is the inverse multiplicative weight. The functions $W_{I M}$ and $G_{O}$ depend on $G$ of (2.27) (this is shown in the Theorem 13 and 14); The $\delta$-uncertainty is obtained from $\alpha$ of (2.27).

$L$ is the function without uncertainty:

$$
L=G_{o} K
$$

this is, nominal plant with controller, and $L_{p}$ of Equation (2.34) is the plant with uncertainty and controller:

$$
L_{p}=G_{p} K
$$

Figure 18: System $L_{p}$ in closed loop.

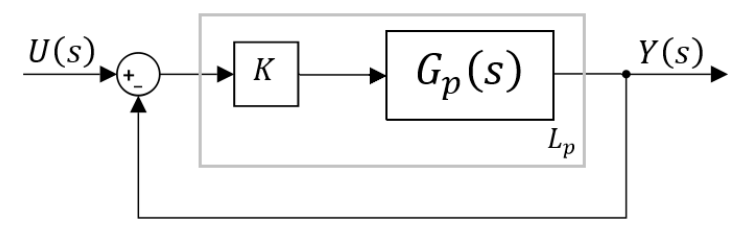

Source: Author.

The transfer function of the system in Figure 18 in closed loop is:

$$
\frac{Y(s)}{U(s)}=\frac{L_{p}(s)}{1+L_{p}(s)}
$$

The next theorem is used to obtain the robustness stability condition.

Theorem 4. The system (2.35) is robustly stable if, and only if,

$$
\left|\frac{W_{I M}}{1+L}\right|<1, \quad \forall \omega
$$


Proof. It can be found in (SKOGESTAD; POSTLETHWAITE, 2007).

Considering $S=\left(I+G_{o} K\right)^{-1}$ the sensitivity function and $T=\left(I+G_{o} K\right)^{-1} G_{o} K$ the complementary sensitivity function, where $G_{o}$ and $K$ are as shown in Figure 17 and Figure 18, respectively, the goal is to design controllers that guarantee the robustness of stability and performance of the system in Figure 18. Let $N$ be a vector, such that: $N=\left[\begin{array}{lll}W_{p} S & W_{u} K S & W_{I M} T\end{array}\right]^{T}$ where $W_{p}, W_{u}$ and $W_{I M}$ are weight functions. The weight $W_{I M}$ is used for modeling the uncertainty and is given by the uncertainty model of the family of plants. The weights $W_{u}$ and $W_{p}$ are chosen by the designer in order to reflect the desired specifications for the closed loop system. $W_{u}$ is used to penalize high controls. According to (SKOGESTAD; POSTLETHWAITE, 2007) the weight could be chosen as:

$$
W_{p}=\frac{s / M+w_{b}}{s+w_{b} E}
$$

where: $\omega_{b}$ is minimum bandwidth frequency (defined as the frequency where $S(j \omega)$ crosses $-3 d B$ from below); $E$ is parameter for $S(s)$ to be small in low frequency, which causes a small stationary error if $E \leq 1 ; M$ is maximum peak magnitude of $S$, that is equal to $\|S(j \omega)\|_{\infty}$. Normally one chooses $M=2$.

Now, consider the condition:

$$
\|N\|_{\infty}=\max _{\omega} \bar{\sigma}(N(j \omega))<1
$$

where $\bar{\sigma}$ is a superior limit to the norm, used here to measure the size of the matrix $N$ at each frequency. For SISO systems, $N$ is a vector and $\bar{\sigma}(N)$ is the usual Euclidean vector norm: $\bar{\sigma}(N)=\sqrt{\left|W_{p} S\right|^{2}+\left|W_{I M} T\right|^{2}+\left|W_{u} K S\right|^{2}}$. Note that the norm shown in (2.38) is different from the norm in (2.7). Because in (2.38) one has a transfer function matrix norm. According to (SKOGESTAD; POSTLETHWAITE, 2007), if the condition (2.38) is satisfied, and the controller $\mathcal{H}_{\infty}(\mathrm{S} / \mathrm{KS} / \mathrm{T})$ is obtained by solving the problem: $\min _{K}\|N(K)\|_{\infty}$, where $K$ is a stabilizing controller, then the robustness of stability and performance of system in Figure 19 is guaranteed.

Note that, a similar procedure can be implemented in the case $(S / K S)$, meaning that the following constraints must be considered:

$$
\|N\|_{\infty}=\max _{\omega} \bar{\sigma}(N(j \omega))<1 ; \quad N=\left[\begin{array}{c}
W_{p} S \\
W_{u} K S
\end{array}\right]
$$


Figure 19: Complete diagram with all weights.

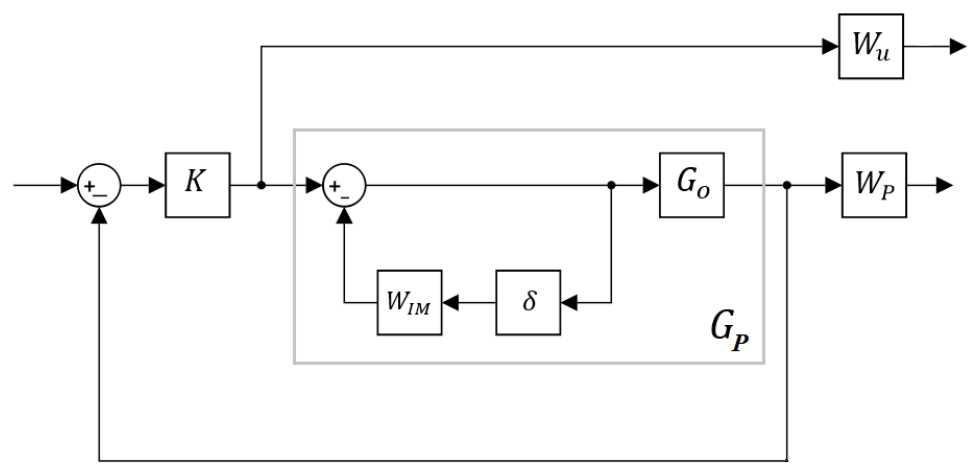

Source: Author.

\subsubsection{Discretization Procedure with Parametric Uncertainties}

For the discretization procedures and obtaining the controllers, a class of Lurie type systems (1.1) with one nonlinearity and the matrix $A$ Hurwitz and for $\sigma \neq 0,0<f(\sigma) \leq$ $k \sigma$, and for $\sigma=0$ one has $f(0)=0$.

Making the assumption of parametric uncertainties in the Lurie system in the discretetime domain, the Zero-Order-Holder $(\mathrm{ZOH})$ is not suitable for the task, due to the fact that the information about the nonlinearity may be lost.

Therefore, for the LP a discretization using the finite difference methods fit perfectly. Also known as the Euler method by difference in forward rectangular. So, a differential equation is transformed into a finite difference equation:

$$
\dot{x}(n) \cong \frac{x[n+1]-x[n]}{T_{s}} .
$$

So, (2.25) in the discrete form becomes:

$$
\left\{\frac{x[n+1]-x[n]}{T_{s}}=A_{\alpha} x[n]+b r_{1}[n],\right.
$$

knowing that $x[n+1]=z X(z), x[n]=X(z)$ and $r_{1}[n]=R_{1}(z)$, one obtains the z-transform of the previous system, its transfer function, and finally the inverse multiplicative input uncertainty:

$$
G_{p}(z)=\frac{X(z)}{R_{1}(z)}=\frac{G(z)}{1+W_{I}(z) \Delta_{I}(z)}, \quad\left\|\Delta_{I}\right\|_{\infty} \leq 1 .
$$

Thereby, the Lurie type system in continuous-time of Figure 4 is modified into the discrete-time system of Figure 20. 
Figure 20: Lurie type system in discrete form.

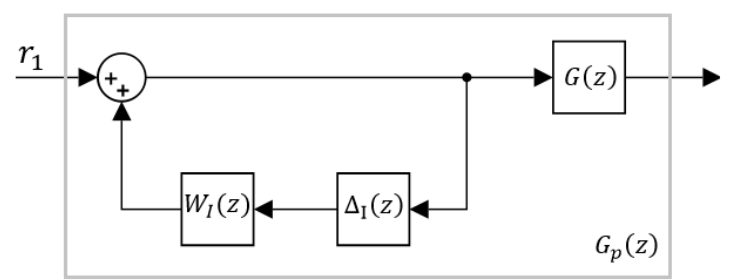

Source: Author.

The $\mathcal{H}_{\infty}$ sub-optimal discrete-time control problem is to find an internally stabilizing controller $K(z)$ such that, for a pre-specified positive value $\gamma$ :

$$
\left\|F_{L}(P, K)\right\|_{\infty}<\gamma
$$

$N$ of the (2.38) or (2.39) is related to $P$ and $K$ by a lower linear fractional transformation, as follows:

$$
F_{L}(P, K)=P_{11}+P_{12} K\left(I-P_{22} K\right)^{-1} P_{21}=N,
$$

in such a way that connects the (2.43) and (2.38) or (2.39). The design of the generalized plant $P$ is explained in detail in (SKOGESTAD; POSTLETHWAITE, 2007). The controller is obtained using the Matlab ${ }^{\circledR}$ functions hinfsyn or mixsyn. These functions obtain the controller solving two Riccati equations in order to satisfy conditions of asymptotic stability for the control problem (see (GU; PETKOV; KONSTANTINOV, 2006)).

\section{6 $\|\mathcal{H}\|_{\infty}$ State Feedback Controller Design via LMI Method}

As mentioned in the introduction, LMIs have a very important place in obtaining new conditions for Lurie problem. In this work, in generally, the results of this thesis do not consist of using LMI, but it will be used a result (Theorems 5) that uses LMI with polytopic uncertainties for comparison. Thus, it is necessary to define LMI. The following definition is adapted from (VANANTWERP; BRAATZ, 2000) and (BIANCHI; BATTISTA; MANTZ, 1981). 
Definition 11. A linear matrix inequality (LMI) has the form:

$$
F(x)=F_{0}+\sum_{i=1}^{m} x_{i} F_{i}>0,
$$

where $x \in \mathbb{R}^{m}, F_{i} \in \mathbb{R}^{n \times n}$ and real symmetric matrices. The inequality means that $F(x)$ is a positive definite matrix, that is,

$$
z^{T} F(x) z>0, \quad \forall z \neq 0, \quad z \in R^{n}
$$

Besides that, it is also possible to define LMIs of the form

$$
\begin{gathered}
F(x)<0, \\
F(x)>G(x),
\end{gathered}
$$

as well as non-strict LMIs such as

$$
F(x) \leq 0
$$

where $F(x)$ and $G(x)$ are affine in $x$. Note that inequalities (2.47) and (2.48) are actually special cases of the (2.45). The matrix inequality (2.47) cab be written as the inequality $-F(x)>0$ and (2.48) as $F(x)-G(x)>0$.

After defining LMI, one can define the control law:

$$
u(m)=K x(m)
$$

In (OLIVEIRA; GEROMEL; BERNUSSOU, 2002), the LMI constraint to design a state feedback controller (2.50) is presented. The controller can be designed using the following theorem:

Theorem 5. There exists a controller in the form (2.50) such that the inequality $\|\mathcal{H}\|_{\infty}^{2}<$ $\xi$ holds if, and only if, the LMI:

$$
\left[\begin{array}{cccc}
P & A X+B L & J & 0 \\
\bullet & X+X^{T}-P & 0 & X^{T} C_{z}^{T}+L^{T} D_{z}^{T} \\
\bullet & \bullet & I & D_{z}^{T} \\
\bullet & \bullet & \bullet & \xi I
\end{array}\right]>0
$$

holds, where the matrices $X$ and $L$ and the symmetric matrix $P$ are the variables. The controller is obtained by $K=L X^{-1}$.

Proof. The proof for this theorem can be found in (OLIVEIRA; GEROMEL; BERNUS- 
SOU, 2002).

\subsubsection{Discretization with Polytopic Uncertainties}

A system subjected to uncertainties can be represented as a polytopic system as follows:

$$
\mathcal{G}_{a}:\left\{\begin{array}{l}
\dot{x}(t)=\mathcal{A} x(t)+\mathcal{B} u(k) \\
y(t)=C(t) x(t)
\end{array}\right.
$$

and if these parameters with uncertainty vary within a known range, it is possible to describe the system matrices as vertex belonging to a polytope. Each vertex represents a linear system with a specific combination of the uncertain parameters.

Consider that the nonlinearity is bounded in a section in the first and third quadrants delimited by a boundary $k$, as presented in the Figure 11. The conversion to a polytopic uncertainties is given by the following equations:

$$
\begin{array}{r}
A_{l}=A+B \rho C, \\
\rho \in[0, k] .
\end{array}
$$

where $A_{l}$ represents the polytope vertex. Generalizing the concept above for a system where there are more than one nonlinearity, the equation to obtain the polytope vertex is given by:

$$
A_{l}=A^{n \times n}+B^{n \times q}\left[\begin{array}{ccc}
\rho_{11} & \ldots & \rho_{1 q} \\
\vdots & \ldots & \vdots \\
\rho_{q 1} & \ldots & \rho_{q q}
\end{array}\right]^{q \times q} \quad C^{q \times n}
$$

With the generalization presented in (2.55) it is possible to create a vertex for each combination of nonlinearity, and also it is possible to consider some coupling between the nonlinearity. After obtaining the vertex the polytope is obtained using the following equations:

$$
\mathcal{A}(\beta)=\left\{\mathcal{A}(\beta)=\sum_{l=1}^{V} \mu_{l} A_{l}, \quad \mu_{l} \geq 0, \sum_{l=1}^{V} \mu_{l}=1\right\} .
$$

It is simple to apply the politope in the LMI constraint shown in the Theorem 5 . The procedure is simply adding the index $l$ in the LMI constraint presented in the Theorem 
5:

$$
\left[\begin{array}{cccc}
P & A_{l} X+B_{l} L & J & 0 \\
\bullet & X+X^{T}-P & 0 & X^{T} C_{z}^{T}+L^{T} D_{z}^{T} \\
\bullet & \bullet & I & D_{z}^{T} \\
\bullet & \bullet & \bullet & \xi I
\end{array}\right]>0
$$

where the addition of the index $l$ means that there are now $l$ LMI constrains, instead of only one constraint, which means that a increase of conservatism in the optimizations problem. For that reason the Theorem 5 loses the necessary condition, and now provides only sub-optimal condition. And, as the number of vertex increases the optimization problem feasibility decreases.

The discretization process is an usual procedure and there are some techniques to perform it, like the $\mathrm{ZOH}$. Yet, the commonly used discretization process imposes some challenges for polytopic systems. That happens as a result of the polytope obtained by discretizing all the continuous-time matrices belonging to a given polytopic system may loose the convexity, therefore, making impossible to use any convex optimization process to design the controllers.

Thus, common procedures like the $\mathrm{ZOH}$ become unfit for the task. However, there are some proposals in the literature to address this challenge, like (SHIEH; WANG; CHEN, 1998) and (HETEL; DAAFOUZ; IUNG, 2007). Those procedures are numerical solutions or Taylor series expansions of the exponential matrices related to the discretization with a finite number of terms. In the present work, one uses the solution provided by (BRAGA et al., 2013), which is a Taylor series expansion with variable number of terms $l$. This feature is desirable, because it is necessary to bound system norm and to the residue norm to ensure the performance. For that, one utilizes the Small Gain Theory. The proof can be found in (GEROMEL; KOROGUI, 2011). Considering the continuous-time polytopic system:

$$
\dot{x}(t)=A_{\text {cont }}(\beta) x(t)+B_{\text {cont }}(\beta) u(t),
$$

the equivalent discrete-time polytopic system is:

$$
x(n+1)=A_{d i s c}(\beta) x(n)+B_{d i s c}(\beta) u(n),
$$

where it is possible to write the discrete matrices as:

$$
\begin{aligned}
& A(\beta)=A_{l}(\beta)+\Delta A_{l}(\beta), \\
& B(\beta)=B_{l}(\beta)+\Delta B_{l}(\beta),
\end{aligned}
$$


where $A_{l}(\beta), B_{l}(\beta)$ are the Taylor series expansion and $\Delta A_{l}(\beta), \Delta B_{l}(\beta)$ Taylor series residue. The matrices $A_{l}(\beta), B_{l}(\beta), \Delta A_{l}(\beta)$ and $\Delta A_{l}(\beta)$ can be calculated as:

$$
\begin{aligned}
A_{l}(\beta) & =\sum_{j=0}^{l} \frac{A_{\text {cont }}(\beta)^{j}}{j !} T^{j}, \\
B_{l}(\beta) & =\sum_{j=1}^{l} \frac{A_{\text {cont }}(\beta)^{j-1}}{j !} T^{j} B_{\text {cont }}(\beta), \\
\Delta A_{l}(\beta) & =e^{A_{\text {cont }}(\beta) T}-A_{l}(\beta), \\
\Delta B_{l}(\beta) & =\left(\int_{0}^{T} e^{A_{\text {cont }}(\beta) s} d s\right) B_{\text {cont }}(\beta)-B_{l}(\beta),
\end{aligned}
$$

where $l \in \mathbb{N}$ is the number of terms in the Taylor expansion and $T>0[\mathrm{~s}]$ is the sampling time. After expanding in Taylor series the polynomial matrices must be homogenized, for details see (BRAGA et al., 2013).

\section{$2.7 \quad \mu$-Analysis and Synthesis}

Developed by Doyle (DOYLE, 1982), the singular structured value is a powerful tool in robust control. The notations normally used for the structured singular value are $\mu$, $\mathrm{mu}$ or SSV. It gives the possibility of obtaining necessary and sufficient conditions for robustness of stability and performance of a closed-loop uncertain system in the form of Figure 22. Here, it is presented a brief summary of Doyle's theory, which in the following sections, enable us to provide sufficient conditions for the Lurie problem.

\subsubsection{General Control Formulation with Uncertainties}

Following the diagram of Figure 6, insert a controller $K(s)$ and replace the functions $f_{j}$ by the uncertain parameters $\alpha_{j}$, as presented in Figure 21.

A general closed-loop control system with uncertainties can be arranged according to Figure 22. Re-arrange the system of Figure 21 in this form, where $P$ is the generalized nominal plant:

$$
P(s)=\left[\begin{array}{lll}
P_{11}(s) & P_{12}(s) & P_{13}(s) \\
P_{21}(s) & P_{22}(s) & P_{23}(s) \\
P_{31}(s) & P_{32}(s) & P_{33}(s)
\end{array}\right] .
$$

By closing the loop of the nominal plant and the controller, one obtains the system 
Figure 21: Lurie type system in closed-loop with controller.

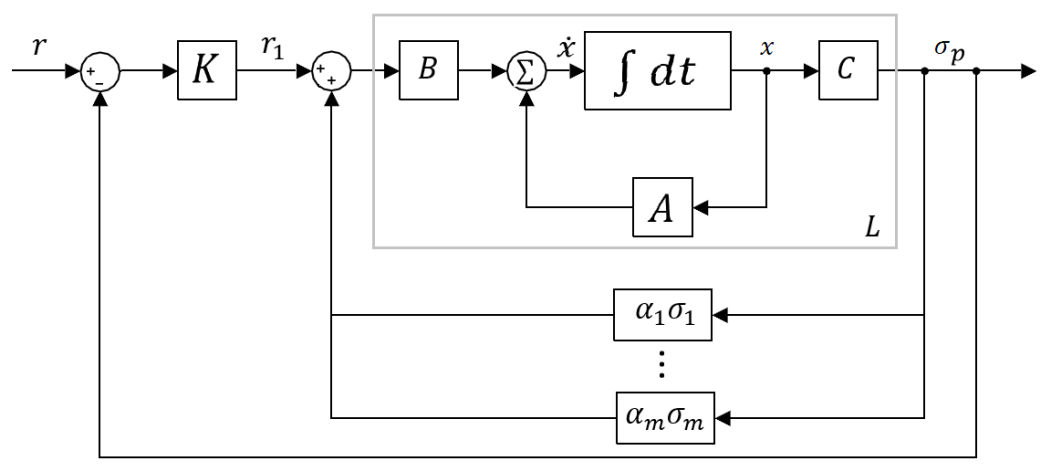

Source: Author.

Figure 22: General Control System.

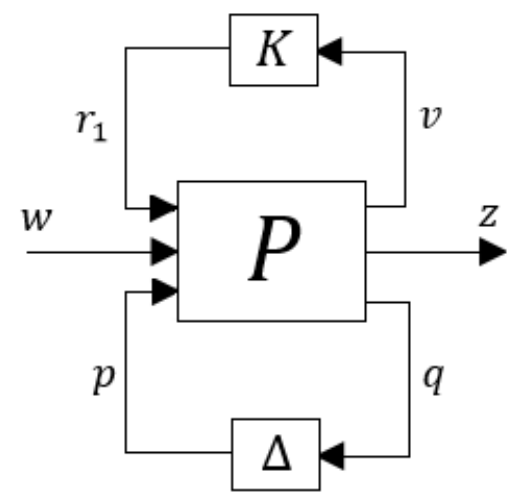

Source: Author.

in Figure 23, such that:

$$
M(s)=F_{U}(P, K)=\left[\begin{array}{ll}
M_{11}(s) & M_{12}(s) \\
M_{21}(s) & M_{22}(s)
\end{array}\right] .
$$

The closed-loop system from $w$ to $z$ is obtained by the lower linear fractional transformation $z=F_{L}(M, \Delta) w$ with:

$$
F_{L}(M, \Delta)=M_{11}+M_{12} \Delta M_{21}\left(I-M_{22} \Delta\right)^{-1}
$$

\subsubsection{Robustness of Stability via $\mu$}

Assume that the system in Figure 23 is nominally stable (i.e., it is stable for $\Delta=0$ ). According to (2.68), the only possibility of instability is in the term $\left(I-M_{22} \Delta\right)^{-1}$. Thus, the stability of the system of Figure 23 is equivalent to the stability of the system $M_{22}$ according to Figure 24. Therefore, one needs to obtain conditions for checking the stability of the $M_{22}$-Structure. The next theorem is derived from the generalized Nyquist theorem. 
Figure 23: $M-\Delta$-Structure.

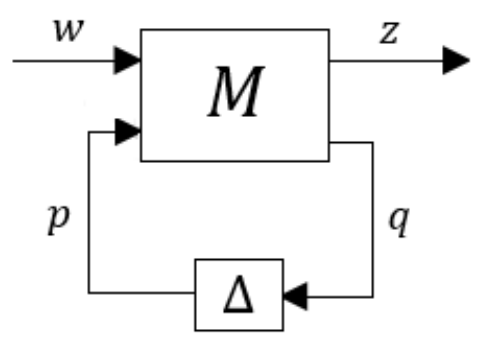

Source: Author.

Theorem 6. (SKOGESTAD; POSTLETHWAITE, 2007). Assume that the nominal system $M_{22}(s)$ is stable. Consider the convex set of perturbations $\Delta$, such that if $\Delta^{\prime}$ is an allowed perturbation then so is $c \Delta^{\prime}$ where $c$ is any real scalar such that $|c| \leq 1$. Then the $M_{22}$-structure is stable for all allowed perturbations (one has $R S$ ) if and only if:

Nyquist plot of $\operatorname{det}\left(I-M_{22} \Delta(s)\right)$ does not encircle the origin, $\forall \Delta$.

$$
\Longleftrightarrow \operatorname{det}\left(I-M_{22} \Delta(j w)\right) \neq 0, \quad \forall w, \forall \Delta
$$

$\Longleftrightarrow \quad \lambda_{i}\left(M_{22} \Delta\right) \neq 0, \quad \forall i, \forall w, \forall \Delta$, where $\lambda_{i}$ are eigenvalues of $M_{22} \Delta$.

Proof. It can be found in (SKOGESTAD; POSTLETHWAITE, 2007).

When $\Delta$ has a block diagonal structure, $\mu$ is a function which provides a generalization of the maximum singular value $\bar{\sigma}$ and the spectral radius $\rho$.

Definition 12. (SKOGESTAD; POSTLETHWAITE, 200\%). The structured singular value, also denoted by $S S V$ or simply $\mu$, is a real non-negative function defined by:

$$
\mu\left(M_{22}\right) \triangleq \frac{1}{\min \left\{h_{m} \mid \operatorname{det}\left(I-h_{m} M_{22} \Delta\right)=0 \text { for structured } \Delta, \bar{\sigma}(\Delta) \leq 1\right\}},
$$

where $M_{22}$ is a complex matrix (matrix of transfer functions), $\Delta=\operatorname{diag}\left\{\Delta_{i}\right\}$ is a block diagonal matrix such that $\bar{\sigma}\left(\Delta_{i}\right) \leq 1$. Some of the blocks may be repeated and some may be restricted to be real. If no such structured $\Delta$ exists, then $\mu\left(M_{22}\right)=0$.

In order to give to the reader a better understanding of the $\mu$ function, remember that the equation $\operatorname{det}\left(I-h_{m} M_{22}(j \omega) \Delta\right)=0$ gives the frequency $\omega$ and the matrices $\Delta$ for which the Nyquist plot is in the borderline of stability (see Theorem 6). Then, $1 / \mu\left(M_{22}\right)$ is the minimal value of $h_{m}$ for which the closed-loop uncertain system is unstable. One can see that this number is related to the stability margin of the closed-loop system. Note that $\mu\left(M_{22}\right)$ is a function of frequency $\omega$. 
Remark 6. In the cases of this work, the matrix $\Delta$ belongs to $\mathbb{R}^{n \times n}$ and is of the form $\Delta=\operatorname{diag}\left\{\delta_{1} I_{n_{1} \times n_{1}}, \delta_{2} I_{n_{2} \times n_{2}}, \cdots, \delta_{m} I_{n_{m} \times n_{m}}\right\}$, where $\delta_{i}$ is real and $n_{1}+n_{2}+\cdots+n_{m}=n$. For different $n$-uples $\left(n_{1}, n_{2}, \cdots, n_{m}\right)$, the value of $\mu$ is different (for the same $\omega$ ).

Remark 7. In general, one cannot find a closed form solution to the optimization problem in (2.72), that is, one has to solve a numerical problem each time. Also, in practical situations one can only find upper and lower bounds to $\mu$. There are several algorithms in the literature for calculating those bounds (GU; PETKOV; KONSTANTINOV, 2006). In particular, the upper bound is a convex problem, which is easier to solve.

In connection with the Remark 7 , for the computation in Matlab ${ }^{\circledR}$, is then defined the upper bound to $\mu$ :

Definition 13 (Upper Bound). The function given by:

$$
m(j \omega)=\min _{D(\omega) \in \mathcal{D}} \bar{\sigma}\left(D(\omega) M(j \omega) D(\omega)^{-1}\right),
$$

where $D \in \mathcal{D}_{\Delta}$ is the set of matrices that commutes with $\Delta$, is the upper bound for $\mu$.

Combining the definition of $\mu$ and condition (2.70) for RS, one can state the following theorem that provides a necessary and sufficient condition for robust stability using $\mu$. This theorem was adapted from (SKOGESTAD; POSTLETHWAITE, 2007).

Theorem 7. Assume that the nominal system $M_{22}$ is stable. Then the $M_{22}-\Delta$-system in Figure 24 is robustly stable for all allowed perturbations with $\bar{\sigma}(\Delta) \leq 1, \forall \omega$, if and only if:

$$
\mu\left(M_{22}(j \omega)\right)<1, \quad \forall w
$$

Proof. It can be found in (SKOGESTAD; POSTLETHWAITE, 2007).

Figure 24: $M_{22}-\Delta$-Structure for robust stability analysis.

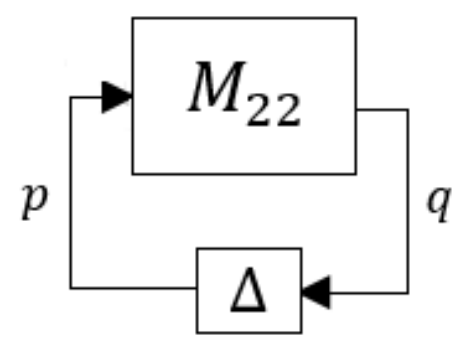

Source: Author. 
Figure 25: $M-\Delta-\bar{\Delta}$-Structure for robust performance analysis.

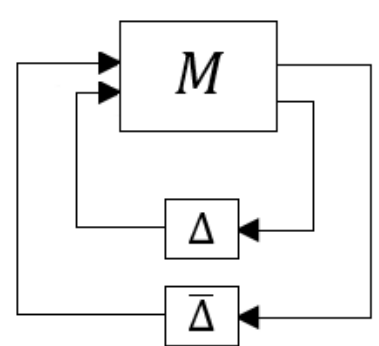

Source: Author.

\subsubsection{Robustness of Performance via $\mu$}

In order to use the $\mu$ tool to the analysis of the robustness of performance, it is common to suppose that the signal $w$ in Figure 23 is generated from the signal $z$ by a uncertain system $\bar{\Delta}(j \omega)$ with $\bar{\sigma}(\bar{\Delta}(j \omega)) \leq 1, \forall \omega$. Then the closed-loop uncertain system assumes the form in Figure 25.

Theorem 8. (SKOGESTAD; POSTLETHWAITE, 2007). Consider the system in Figure 25, and assume that the nominal system $M$ is (internally) stable. Then the system has robustness of performance if and only if:

$$
\mu\left(M_{\tilde{\Delta}}(j \omega)\right)<1, \quad \forall \omega
$$

where $\mu$ is computed with respect to the matrix:

$$
\tilde{\Delta}=\left[\begin{array}{ll}
\Delta & 0 \\
0 & \bar{\Delta}
\end{array}\right],
$$

and $\bar{\Delta}$ is a full complex transfer function matrix with the same dimensions as $F_{L}(M, \Delta)^{T}$.

Proof. It can be found in (SKOGESTAD; POSTLETHWAITE, 2007).

\subsection{4 $\mu$-Analysis for Systems with Delay}

In order to determine the stability of delayed systems, two types of analysis can be performed. One is when the stability of the system is not delay-dependent, this being called delay-independent stability. The other is when the stability depends on delay. In general, for the first case, the conditions are more conservative. Therefore, in this work, the second type will be treated, that is, delay-dependent stability. This section is based on the theories of (HUANG; ZHOU, 2000) and (WANG; LUNDSTROM; SKOGESTAD, 1994), where it is possible to determine the stability for delay-dependent systems via 
$\mu$-analysis.

Consider a time-delay system:

$$
\dot{x}=A x(t)+\sum_{i=1}^{k} A_{i} x\left(t-\tau_{i}\right),
$$

where $\tau_{i} \in\left[0, h_{i}\right], i=1,2, \ldots, k$ are uncertain constants, such that $h_{i} \geq 0$ for $i>j$, with initial condition $x\left(t_{0}+\theta\right)=\phi(\theta), \forall \theta \in[-\bar{\tau}, 0], \bar{\tau}=\max _{i}\left(\tau_{i}\right)$.

Considering that the analysis will be of the type delay-dependent stability, an alternative representation of the previous system is required. Notice that system (2.77) can be rewritten as

$$
\dot{x}=\left(A+\sum_{i=1}^{k} A_{i}\right) x(t)+\sum_{i=1}^{k} A_{i}\left(x\left(t-\tau_{i}\right)-x(t)\right) .
$$

For convenience, one denotes $\mathcal{D}_{\tau}$ the delay operator such that

$$
\mathcal{D}_{\tau} \phi(t)=\phi(t-\tau)
$$

for any scalar function $\phi(t)$.

In addition, assume that there is a $B_{i} \in \mathbb{R}^{n x r_{i}}$ and a $C_{i} \in \mathbb{R}^{r_{i} x n}$ such that

$$
A_{i}=B_{i} C_{i}
$$

Thus, an alternative interconnected form for the system (2.78) is

$$
\left\{\begin{array}{l}
\dot{x}(t)=\left(A+\sum_{i=1}^{k} A_{i}\right) x(t)+\sum_{i=1}^{k} B_{i} u_{i}(t) \\
y_{i}=C_{i} x(t) \\
u_{i}(t)=\left(\mathcal{D}_{\tau_{i}}-1\right) I_{r_{i}} y_{i}(t)
\end{array}\right.
$$

and shown in Figure 26.

So,

$$
P(s)=C\left(s I-A-\sum_{i=1}^{k} A_{i}\right)^{-1} B .
$$

Since the magnitude of uncertainty

$$
\Delta=\operatorname{diag}\left(\left(e^{-\tau_{1} s}-1\right) I_{\tau_{1}}, \ldots,\left(e^{-\tau_{k} s}-1\right) I_{\tau_{k}}\right.
$$


Figure 26: Uncertain delay system.

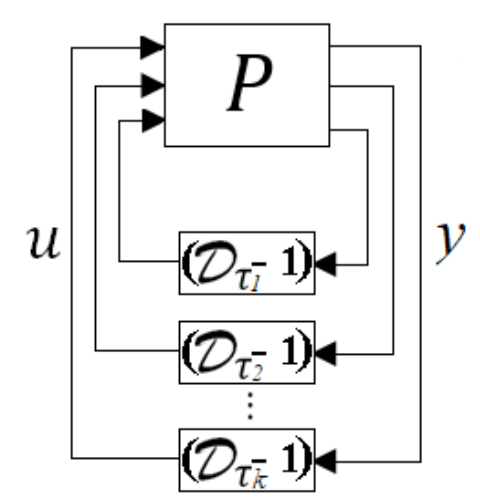

Source: Author.

is bounded by

$$
W(w)=\operatorname{diag}\left(\gamma_{1}(w) I_{\tau_{1}}, \ldots, \gamma_{k}(w) I_{\tau_{k}}\right),
$$

where $\gamma_{i}(w)$ bounds the magnitude of $e^{-j w \tau_{i}}-1$.

A number of possible functions that satisfy $\gamma(\omega) \leq\left\|\gamma_{i, j}^{*}(s)\right\|, j=1,2,3,4,5$, are provided by (WANG; LUNDSTROM; SKOGESTAD, 1994):

$$
\begin{gathered}
\gamma_{i, 1}^{*}(s)=\tau_{i} s, \\
\gamma_{i, 2}^{*}(s)=\frac{\tau_{i} s}{\tau_{i} s / 3.465+1}, \\
\gamma_{i, 3}^{*}(s)=\frac{1.216 \tau_{i} s}{\tau_{i} s / 2+1}, \\
\gamma_{i, 4}^{*}(s)=\frac{\tau_{i} s\left(2 x 0.2152^{2} \tau_{i} s+1\right.}{\left(0.2152 \tau_{i} s+1\right)^{2}}, \\
\gamma_{i, 5}^{*}(s)=\frac{\tau_{i} s}{\tau_{i} s / 2+1} \frac{\left(\tau_{i} s / 2.363\right)^{2}+1.676\left(\tau_{i} s / 2.363\right)+1}{\left(\tau_{i} s / 2.363\right)^{2}+1.370\left(\tau_{i} s / 2363\right)+1} .
\end{gathered}
$$

In Figure 27 is shown the frequency response of these transfer functions.

The following theorem provides a sufficient condition for delayed system via $\mu$-analysis.

Theorem 9. The uncertain delay system (2.77) is robustly stable for all $\tau_{i} \in\left[0, \tau_{i}\right)$ if $\dot{x}=\left(A+\sum_{i=1}^{k} A_{i}\right) x(t)$ is stable and:

$$
\mu_{\Delta}(W(\omega) P(j \omega))<1, \quad \forall \omega
$$


Figure 27: Frequency response of $\gamma_{i}$ and $\gamma_{i, j}^{*}$.



Source: Author.

Proof. See (HUANG; ZHOU, 2000).

\subsection{5 $\mu$-Synthesis via DK-Iteration}

The DK-iteration, proposed by Doyle (DOYLE, 1985), is a synthesis method of robust controllers that combines $\mathcal{H}_{\infty}$ design with $\mu$-analysis. The method is based upon a property that relates the upper bound of $\mu$ with the scaled singular value (SKOGESTAD; POSTLETHWAITE, 2007) as defined by Definition 13, that is:

$$
\mu(M) \leq \min _{D \in \mathcal{D}} \bar{\sigma}\left(D M D^{-1}\right)=m(j \omega)
$$

where $\mathcal{D}$ is the set of matrices that commute with $\Delta$, and $M=F_{U}(P, K)$ according to (2.67). It can be shown that the minimization problem in (2.86) is convex (GU; PETKOV; KONSTANTINOV, 2006). Therefore, the goal of the DK-iteration is to find some controller $K(s)$ that minimizes the peak value of the upper bound of $\mu(M)$ as follows:

$$
\min _{K(s)}\left(\min _{D \in \mathcal{D}}\left\|D F_{U}(P, K) D^{-1}\right\|_{\infty}\right)
$$

Briefly, a suggestion of DK-iteration can be seen in Algorithm 1. 


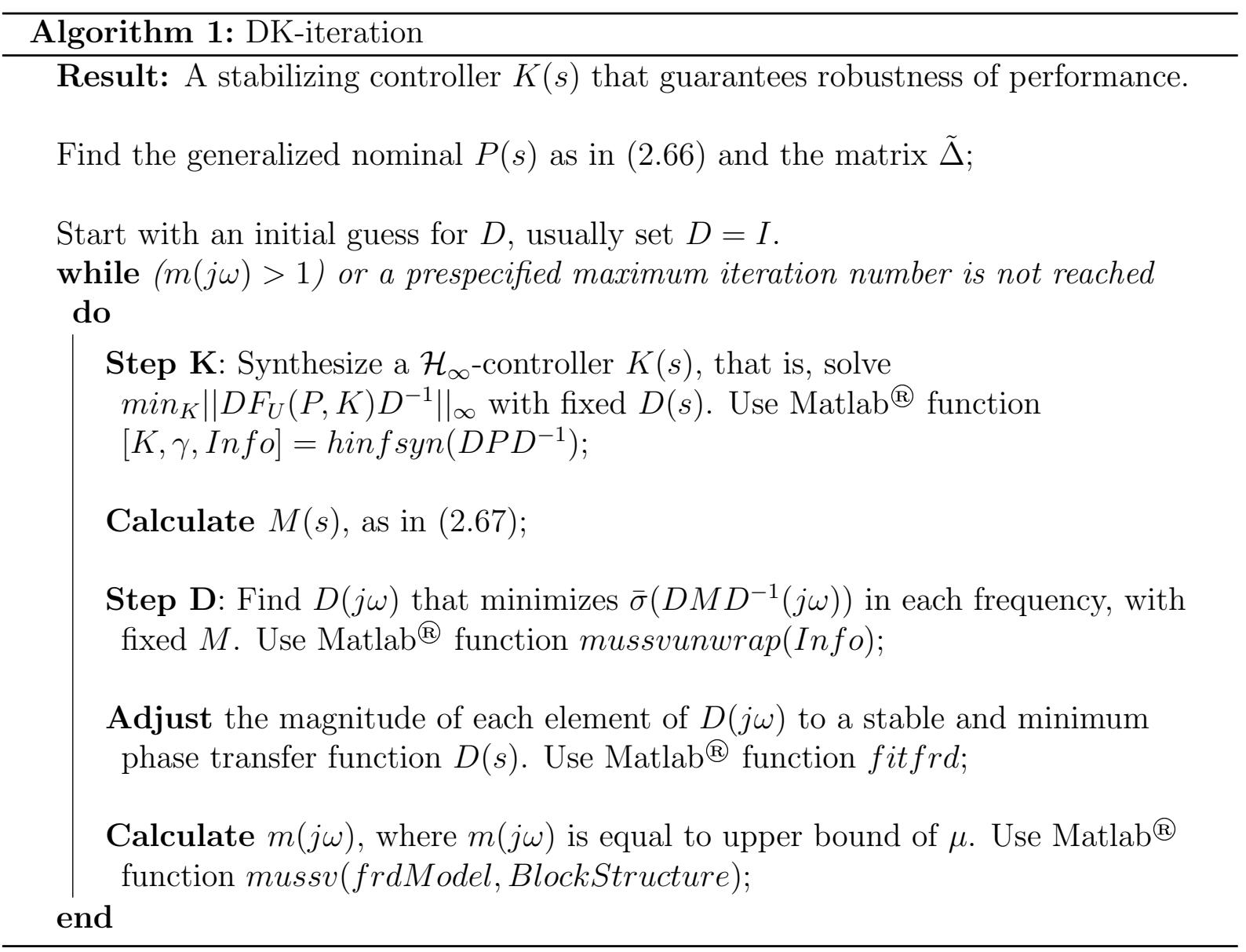

Remark 8. A method to find the generalized plant $P(s)$ can be found in (SKOGESTAD; POSTLETHWAITE, 2007; GU; PETKOV; KONSTANTINOV, 2006). It is worth mentioning that $P(s)$ must include the performance specifications. In this work, it is applied to Lurie type systems.

Remark 9. The Matlab ${ }^{\circledR}$ function mussv must receive the information about the structure of matrix $\Delta$ in the following form:

If the $i$-th block is $r$-by-r block diagonal with repeated real scalar perturbation, then use

$$
\text { BlockStructure }(i,:)=\left[\begin{array}{ll}
-r & 0
\end{array}\right] \text {. }
$$

If the $i$-th block is $r$-by-r block diagonal with repeated complex scalar perturbation, then use

$$
\text { BlockStructure }(i,:)=\left[\begin{array}{ll}
r & 0
\end{array}\right] .
$$


If the $i$-th block is an r-by-c of complex full-block perturbation, then

$$
\begin{aligned}
& \text { BlockStructure }(i,:)=\left[\begin{array}{ll}
r & c
\end{array}\right] \text {. } \\
& \text { For , the Matlab }{ }^{\circledR} \text { form of } \Delta=\left[\begin{array}{ccccc}
\delta_{1} & 0 & 0 & 0 & 0 \\
0 & \delta_{2} & 0 & 0 & 0 \\
0 & 0 & \delta_{2} & 0 & 0 \\
0 & 0 & 0 & \delta_{3} & \delta_{4} \\
0 & 0 & 0 & \delta_{5} & \delta_{6}
\end{array}\right] \text {, for } \delta_{j} \in \mathbb{C} i s \text { : } \\
& \text { BlockStructure }=\left[\begin{array}{ll}
1 & 0 \\
2 & 0 \\
2 & 2
\end{array}\right] \text {. }
\end{aligned}
$$

\subsection{Padé Approximation}

\subsubsection{Classical Formulation of Padé Approximation}

Given an analytic function on a region of $\mathbb{C}$ given by a power series:

$$
f(s)=\sum_{i=0}^{\infty} c_{i} s^{i},
$$

a Padé approximation of order $(L, M)$ is a rational function given by:

$$
[L / M]=\frac{a_{0}+a_{1} s+\ldots+a_{L} s^{L}}{b_{0}+b_{1} s+\ldots+b_{M} s^{M}} .
$$

Thus, the following approximation is valid: 


$$
\sum_{i=0}^{\infty} c_{i} s^{i}=\frac{a_{0}+a_{1} s+\ldots+a_{L} s^{L}}{b_{0}+b_{1} s+\ldots+b_{M} s^{M}}+O\left(s^{L+M+1}\right),
$$

and cross-multiplying is found:

$$
\left(b_{0}+b_{1} s+\ldots+b_{M} s^{M}\right)\left(c_{0}+c_{1} s \ldots\right)=a_{0}+a_{1} s+\ldots+a_{L} s^{L}+O\left(s^{L+M+1}\right),
$$

such that the coefficients $b^{\prime} s$ can be found by solving the linear system:

$$
\left[\begin{array}{ccccc}
c_{L-M+1} & c_{L-M+2} & c_{L-M+3} & \cdots & c_{L} \\
c_{L-M+2} & c_{L-M+3} & c_{L-M+4} & \cdots & c_{L+1} \\
c_{L-M+3} & c_{L-M+4} & c_{L-M+5} & \cdots & c_{L+2} \\
\vdots & \vdots & \vdots & \ddots & \vdots \\
c_{L} & c_{L+1} & c_{L+2} & \cdots & c_{L+M-1}
\end{array}\right]\left[\begin{array}{l}
b_{M} \\
b_{M-1} \\
b_{M-2} \\
\vdots \\
b_{1}
\end{array}\right]=-\left[\begin{array}{l}
c_{L+1} \\
c_{L+2} \\
c_{L+3} \\
\vdots \\
c_{L+M}
\end{array}\right]
$$

and the coefficients can be found by the formula:

$$
\begin{aligned}
& a_{0}=c_{0}, \\
& a_{1}=c_{1}+b_{1} c_{0}, \\
& a_{2}=c_{2}+b_{1} c_{1}+b_{2} c_{0}, \\
& \vdots \\
& a_{L}=c_{L}+\sum_{i=1}^{\min (L, M)} b_{i} c_{L-i} .
\end{aligned}
$$

\subsubsection{Modern Formulation of the Padé Approximation}

Let be the determinants:

$$
P^{[L / M]}(s)=\left|\begin{array}{cccc}
c_{L-M+1} & c_{L-M+2} & \cdots & c_{L+1} \\
c_{L-M+2} & c_{L-M+3} & \cdots & c_{L+2} \\
\vdots & \vdots & \ddots & \vdots \\
c_{L-1} & c_{L} & \cdots & c_{L+M-1} \\
c_{L} & c_{L+1} & \cdots & c_{L+M} \\
\vdots & \vdots & \ddots & \vdots \\
\sum_{i=0}^{L-M} c_{i} s^{M+i} & \sum_{i=0}^{L-M+1} c_{i} s^{M+i-1} & \cdots & \sum_{i=0}^{L} c_{i} s^{i}
\end{array}\right|
$$

and 


$$
Q^{[L / M]}(s)=\left|\begin{array}{ccccc}
c_{L-M+1} & c_{L-M+2} & \cdots & c_{L} & c_{L+1} \\
c_{L-M+2} & c_{L-M+3} & \cdots & c_{L+1} & c_{L+2} \\
\vdots & \vdots & \ddots & \vdots & \vdots \\
c_{L-1} & c_{L} & \cdots & c_{L+M-2} & c_{L+M-1} \\
c_{L} & c_{L+1} & \cdots & c_{L+M-1} & c_{L+M} \\
s^{M} & s^{M-1} & \cdots & s & 1
\end{array}\right|
$$

Then, another way to write Padé's approximation of $\sum_{i=0}^{\infty} c_{i} s^{i}$ is defined:

$$
[L / M]=\frac{P^{[L / M]}(s)}{Q^{L / M}(s)},
$$

provided $Q^{L / M}(s) \neq 0$.

So that:

$$
\sum_{i=0}^{\infty} c_{i} s^{i}-\frac{P^{[L / M]}(s)}{Q^{L / M}(s)}=O\left(s^{L+M+1}\right)
$$

\subsubsection{Padé Approximation of the Exponential Function}

For the case of the exponential function, one has:

$$
Q^{[L / M]}(s)=\left|\begin{array}{ccccc}
\frac{1}{(L-M+1) !} & \frac{1}{(L-M+2) !} & \cdots & \frac{1}{L !} & \frac{1}{(L+1) !} \\
\frac{1}{(L-M+2) !} & \frac{1}{(L-M+3) !} & \cdots & \frac{1}{(L+1) !} & \frac{1}{(L+2) !} \\
\vdots & \vdots & \ddots & \vdots & \vdots \\
\frac{1}{L !} & \frac{1}{(L+1) !} & \cdots & \frac{1}{(L+M-1) !} & \frac{1}{(L+M) !} \\
s^{M} & s^{M-1} & \cdots & s & 1
\end{array}\right| .
$$

One can further simplify it as follows:

$$
C[L / M]=\left|\begin{array}{cccc}
\frac{1}{(L-M+1) !} & \frac{1}{(L-M+2) !} & \cdots & \frac{1}{L !} \\
\frac{1}{(L-M+2) !} & \frac{1}{(L-M+3) !} & \cdots & \frac{1}{(L+1) !} \\
\vdots & \vdots & \ddots & \vdots \\
\frac{1}{L !} & \frac{1}{(L+1) !} & \cdots & \frac{1}{(L+M-1) !}
\end{array}\right| .
$$

And: 


$$
\begin{gathered}
Q^{[L / M]}(s)=C(L / M) \sum_{j=0}^{M} \frac{(L+M-j) !}{(L+M) !} \frac{M !}{(M-j) !} \frac{(-s)^{j}}{j !} \\
P^{[L / M]}(s)=C(L / M) F(-L,-L-M ; s)
\end{gathered}
$$

so that:

$$
[L / M]=\frac{F(-L,-L-M ; s)}{F(-M,-L-M ; s)}
$$

where

$$
F(-M,-L-M ; s)=\sum_{j=0}^{M} \frac{(L+M-j) !}{(L+M) !} \frac{M !}{(M-j) !} \frac{(-s)^{j}}{j !}
$$

For the case of the pure exponential function, it can be shown that:

Table 2: Padé table of $\exp (\mathrm{s})$.

\begin{tabular}{|c|c|c|c|}
\hline$M \backslash L$ & 0 & 1 & 2 \\
\hline 0 & $\frac{1}{1}$ & $\frac{1+s}{1}$ & $\frac{2+2 s+s^{2}}{2}$ \\
1 & $\frac{1}{1-s}$ & $\frac{2+s}{2-s}$ & $\frac{6+4 s+s^{2}}{6-2 s}$ \\
2 & $\frac{2}{2-2 s+s^{2}}$ & $\frac{6+2 s}{6-4 s+s^{2}}$ & $\frac{12+6 s+s^{2}}{12-6 s+s^{2}}$ \\
\hline
\end{tabular}

Source: Author.

\subsubsection{Theorems Useful for Padé Approximation}

The following theorem, known as Homographic Invariant of Value Transformations, allows to calculate Padé approximations for transformations of simple functions, given that the Padé approximation of the original function is known.

Theorem 10. Given a function $f(s)=\sum_{i=0}^{\infty} c_{i} s^{i}$, one defines

$$
g(s)=\frac{a+b f(s)}{c+d f(s)}
$$

If $c+d f(0) \neq 0$, then

$$
[M / M]_{g}(s)=\frac{a+b[M / M]_{f}(s)}{c+d[M / M]_{f}(s)}
$$

provided $[M / M]_{f}(s)$ exists.

Proof. See (BAKER; GRAVE-MORRIS, 1981).

The following theorem proves the uniform convergence of Padé's approximation. 
Theorem 11. Let $f(s)$ be a meromorphic function. Suppose that $\epsilon$ and $\delta$ are given positive numbers. Then $M_{0}$ exists such that any $[M / M]$ Padé approximant satifies:

$$
\|f(s)-[M / M]\|<\epsilon
$$

for all $M>M_{0}$ on any compact set of s-plane except for a set $\mathcal{E}_{M}$ of measure less than $\delta$.

Proof. See (BAKER; GRAVE-MORRIS, 1981).

The reader should note that this convergence is uniform, as $\delta$ and $\epsilon$ do not depend on $s$.

\subsubsection{Some Concepts for Rational Matrices and Complex Func- tions}

Let $A_{1}, A_{2}, \ldots$ be a sequence of matrices belonging to $\mathbb{C}^{m \times n}$ and let $a_{i j}^{(n)}$ be the $i, j$ th element of $A_{p}, p=1,2, \ldots$. The sequence $A_{1}, A_{2}, \ldots$ is said to converge to the matrix $A \in \mathbb{C}^{m \times n}$ if there exist numbers $a_{i j}$ (the elements of $A$ ) such that $a_{i j}^{(p)} \rightarrow a_{i j}$ as $p \rightarrow \infty$ for each pair of subscripts $i, j$. A sequence that does not converge is defined as elementwise convergence. The definition also includes the convergence of column and row vectors as special cases (LANCASTER; TISMENETSKY, 1985).

Since each matrix component converges uniformly, it is natural that the sequence of matrices also converges uniformly. Furthermore, the interest here is in uniform convergence of functions, the following definition and theorem (Weierstrass's theorem) are important.

Definition 14. Given a sequence of functions $\left\{f_{n}(s)\right\}$, which converges to $f(s)$, this sequence is said to converge uniformly if for each $\epsilon>0$, there exists a positive integer $N$ such that for all $n>N$ one has:

$$
\left|f_{k}(s)-f(s)\right|<\epsilon
$$

Remark 10. In the case of the sequence of partial sums $S_{n}=\sum_{i=0}^{n} s^{i}$, it does not converge uniformly at $|s|<1$, however, for any $k<1$, it converges uniformly to $|s| \leq k$.

Theorem 12 (Weierstrass). If each member of a sequence of functions $f_{1}(s), f_{2}(s)$, $\cdots, f_{n}(s)$ is analytic in $D \subset \mathbb{C}$ and converges uniformily to a function $f(s)$ in any domain $S$ in the interior of $D$, then $f(s)$ is analytic in $S$. 
Proof. See (BAKER; GRAVE-MORRIS, 1981). 


\section{SUFFICIENT CONDITIONS IN SISO CASE}

In this section, the results are divided into four parts. First, two sufficient conditions are presented for the original Lurie problem in (1.1), which are Theorems 13 and 14 . Second, it is obtained a condition for time-delay systems and it is applied in the Chua's circuit. Third, the synthesis of a controller using Theorem 13 and $\mathcal{H}_{\infty}$ Mixed-Sensitivity $(\mathrm{S} / \mathrm{KS} / \mathrm{T})$ and $(\mathrm{S} / \mathrm{KS})$ is presented in Theorems 16 and 17. Fourth, an initial approach of the synthesis of a controller is made to the discrete case in SISO systems. The results presented concern to the class of $f$ functions such that $f \in F_{(0, k]}$ and $f \in F_{\left[k_{1}, k_{2}\right]}$.

\subsection{Sufficient Conditions}

In order to use the criterion of the theory of robust stability presented by the condition (2.36), consider the following lemma:

Lemma 3. The system (2.35) is equivalent to the system (2.27), if:

$$
G_{p}(s)=\frac{G(s)}{K[1+G(s)(\alpha-1)]} .
$$

Figure 28: Equivalent systems by Lemma 3.

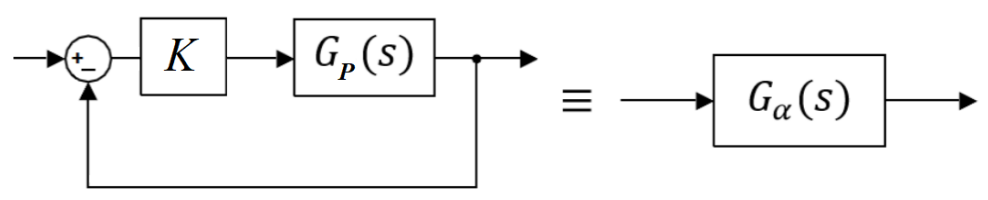

Source: Author.

Proof. In order to have equivalence according to Figure 28, one must do:

$$
\frac{L_{p}(s)}{1+L_{p}(s)}=G_{\alpha}(s)
$$

Isolating $L_{p}(s)$, one has:

$$
L_{p}(s)=\frac{G_{\alpha}(s)}{1-G_{\alpha}(s)}
$$


But, of (2.34), one has $L_{p}=K G_{p}$, so:

$$
G_{p}(s)=\frac{G_{\alpha}(s)}{K\left[1-G_{\alpha}(s)\right]}
$$

Replacing the system (2.27) in (3.2), one has (3.1).

Theorem 13. Suppose that $f \in F_{(0, k]}$ and assume that the nominal system $G_{p}(s)$ in closed loop is stable. Then the system (1.1) is absolutely stable for some class of functions $f \in F_{(0, k]}$ if:

$$
\left|\frac{\frac{k}{2} G_{o}(j w)}{1+G_{o}(j w)}\right|<1, \quad \forall \omega
$$

where:

$$
G_{o}(s)=\frac{G(s)}{1-G(s)+G(s) \frac{k}{2}},
$$

and

$$
G(s)=c^{T}(s I-A)^{-1} b .
$$

Proof. The aim is to transform the problem of the absolute stability of the system (1.1) in the robust stability problem of the system (2.35). This system is obtained as follows: By Lemma 3 and making the controller $K=1$, one has:

$$
G_{p}(s)=\frac{G(s)}{1+G(s)(\alpha-1)}=\frac{G(s)}{1+G(s) \alpha-G(s)},
$$

since one has $f \in F_{(0, k]}$ one must do $\alpha=\frac{k}{2}+\frac{k}{2} \delta,|\delta| \leq 1$. So:

$$
G_{p}(s)=\frac{G(s)}{1+G(s) \frac{k}{2}+G(s) \frac{k}{2} \delta-G(s)},
$$

dividing the numerator and denominator by $1+G(s) \frac{k}{2}-G(s)$, one has:

$$
G_{p}(s)=\frac{\frac{G(s)}{1+G(s) \frac{k}{2}-G(s)}}{1+\left(\frac{G(s) \frac{k}{2}}{1+G(s) \frac{k}{2}-G(s)}\right) \delta}
$$

so one has inverse multiplicative input uncertainty, according to $(2.32): G_{p}(s)=\frac{G_{o}(s)}{1+W_{I M}(s) \delta}$, $|\delta| \leq 1$. Where:

$$
\begin{gathered}
G_{o}(s)=\frac{G(s)}{1-G(s)+G(s) \frac{k}{2}} \\
W_{I M}(s)=G_{o}(s) \frac{k}{2} \quad \text { and } \quad L=G_{o} K=G_{o} .
\end{gathered}
$$

By applying the Theorem 4, which leads us to $\left|\frac{\frac{k}{2} G_{o}(j w)}{1+G_{o}(j w)}\right|<1$, and by Lemma 1 the 
theorem is proved.

Theorem 14. Suppose that $f \in F_{\left[k_{1}, k_{2}\right]}$ and assume that the nominal system $G_{p}^{k}(s)$ in closed loop is stable. Then the system (1.1) is absolutely stable for some class of functions $f \in F_{\left[k_{1}, k_{2}\right]}$ if:

$$
\left|\frac{\left(\frac{k_{2}-k_{1}}{2}\right) G_{o}^{k}(j w)}{1+G_{o}^{k}(j w)}\right|<1, \quad \forall \omega
$$

where:

$$
G_{o}^{k}(s)=\frac{G(s)}{1-G(s)+G(s)\left(\frac{k_{2}+k 1}{2}\right)},
$$

and

$$
G(s)=c^{T}(s I-A)^{-1} b .
$$

Proof. The proof of this theorem is analogous to the Theorem 13, with a different detail: one must do $k_{1} \leq \alpha \leq k_{2}$, thus:

$$
\begin{gathered}
\alpha=k_{1}+\left(\frac{k_{2}-k_{1}}{2}\right)+\frac{\left(k_{2}-k_{1}\right)}{2} \delta=\left(\frac{k_{2}+k_{1}}{2}\right)+\frac{\left(k_{2}-k_{1}\right)}{2} \delta, \\
|\delta| \leq 1, \quad \text { for } \quad j=1, \ldots, m .
\end{gathered}
$$

So,

$$
G_{p}^{k}(s)=\frac{G(s)}{1+G(s)\left(\frac{k_{2}+k_{1}}{2}\right)+G(s)\left(\frac{k_{2}-k_{1}}{2}\right) \delta-G(s)},
$$

dividing the numerator and denominator by $1+G(s)\left(\frac{k_{2}+k_{1}}{2}\right)-G(s)$, one has:

$$
G_{p}^{k}(s)=\frac{\frac{G(s)}{1+G(s)\left(\frac{k_{2}+k_{1}}{2}\right)-G(s)}}{1+\left(\frac{G(s)\left(\frac{k_{2}-k_{1}}{2}\right)}{1+G(s)\left(\frac{k_{2}+k_{1}}{2}\right)-G(s)}\right) \delta}
$$

so, one has inverse multiplicative input uncertainty, according to (2.32):

$$
G_{p}^{k}(s)=\frac{G_{o}^{k}(s)}{1+W_{I M}^{k}(s) \delta}, \quad|\delta| \leq 1 .
$$

Where:

$$
\begin{gathered}
G_{o}^{k}(s)=\frac{G(s)}{1+G(s)\left(\frac{k_{2}+k_{1}}{2}\right)-G(s)}, \\
W_{I M}^{k}(s)=G_{o}^{k}(s)\left(\frac{k_{2}+k_{1}}{2}\right) \quad \text { and } L=G_{o}^{k} K=G_{o}^{k} .
\end{gathered}
$$

Using the condition $(2.36)$ of the Theorem 4, which leads us to $\left|\frac{\left(\frac{k_{2}-k_{1}}{2}\right) G_{o}^{k}(j w)}{1+G_{o}^{k}(j w)}\right|<1$, and by Lemma 2 the theorem is proved. 
Example 1. Check the stability of the Lurie type system below, in sector $f \in F_{(0,1]}$ :

$$
\left\{\begin{array}{c}
\dot{x_{1}}=-2 x_{1}+x_{2}+2 f\left(x_{1}+x_{2}\right) \\
\dot{x_{2}}=-x_{1}-x_{2}+f\left(x_{1}+x_{2}\right)
\end{array}\right.
$$

In this case, one has:

$$
A=\left(\begin{array}{cc}
-2 & 1 \\
-1 & -1
\end{array}\right), b=\left(\begin{array}{l}
2 \\
1
\end{array}\right), c^{T}=\left(\begin{array}{ll}
1 & 1
\end{array}\right) .
$$

Using the Theorem 13, it is found a maximum value for $k, k_{\max }=0.8677$. Easily it is verified which the nominal systems is stable, so in Figure 29 the condition (3.3) is satisfied (note that $0 d B=$ unitary gain). Therefore, the system is absolutely stable for $0 \leq k \leq 0.8677$.

In addition, the Popov's criterion is used, with $q=10^{7}$ to compare with this result. In Figure 30, with $k=0.8662$, the Popov's criterion is not satisfied. The $k$ value to ensure absolute stability by Popov's criterion should be $k=0.8661$. The developed criterion supplies a condition slightly more relaxed lower than Popov's criterion, probably due to numerical precision

Figure 29: Conditon $\left|\frac{\frac{k}{2} G_{o}(j w)}{1+G_{o}(j w)}\right|<1, \forall \omega$ satisfied for $\mathrm{k}=0.8677$.

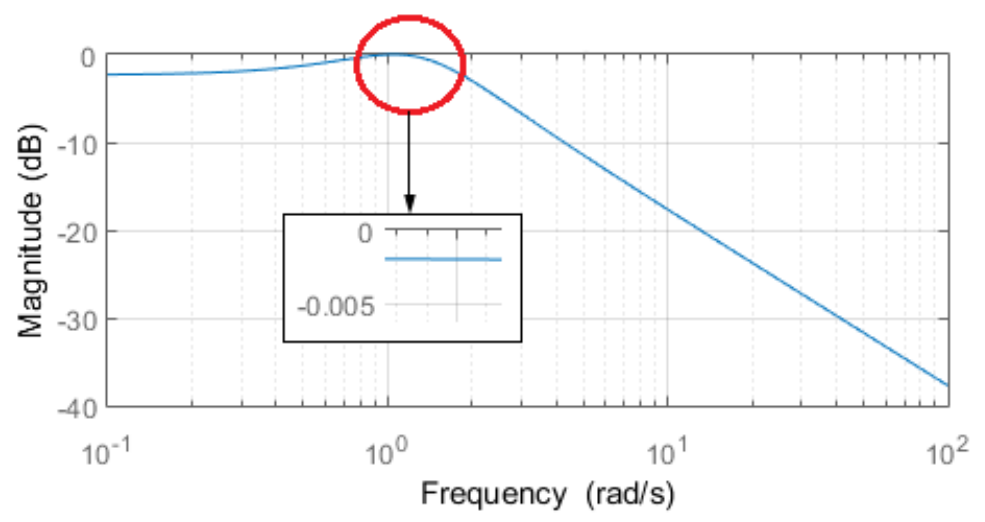

Source: Author. 
Figure 30: Popov's method for $k=0.8662$.

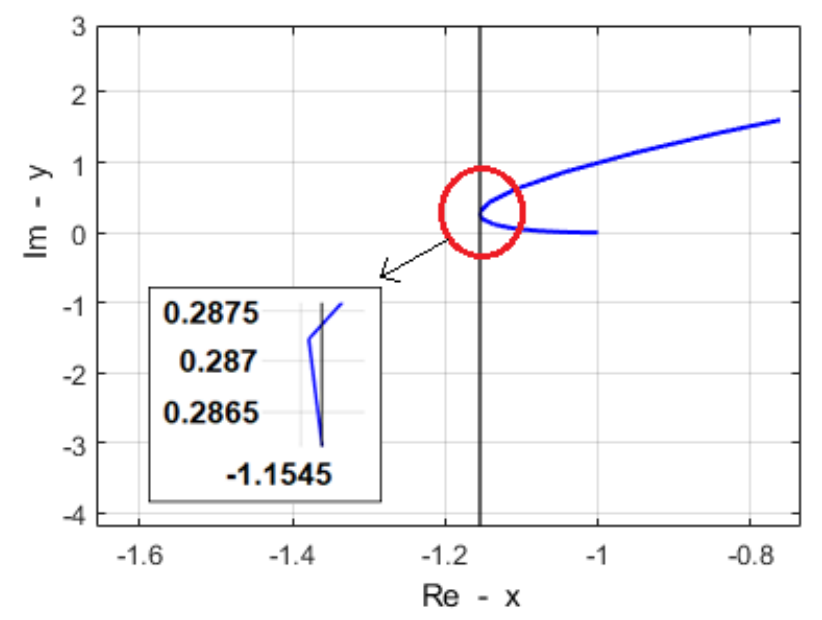

Source: Author.

\subsection{Time-delay SISO Lurie type systems}

Let be the Lurie type system with delay according to the following equation

$$
\left\{\begin{array}{l}
\dot{x}=A x-b f(\sigma)+F_{\tau} x(t-\tau)+b r_{1}, \\
\sigma=c^{T} x
\end{array}\right.
$$

where $x \in \mathbb{R}^{n}$ is the state vector, $\tau>0$ is a real constant delay, $f(\sigma) \in \mathbb{R}$ and $\sigma \in \mathbb{R}$. One has the fixed matrices $F_{\tau} \in \mathbb{R}^{n \times n}, b \in \mathbb{R}^{n}, c \in \mathbb{R}^{n}$ and $A \in \mathbb{R}^{n \times n}$ is Hurwitz. The equation (3.9) is an extension of equation (1.1), which includes the time-delay. To illustrate the insertion of the delay in system (1.1), let's consider the Figure 31.

Figure 31: Block diagram of the Lurie type system with delay.

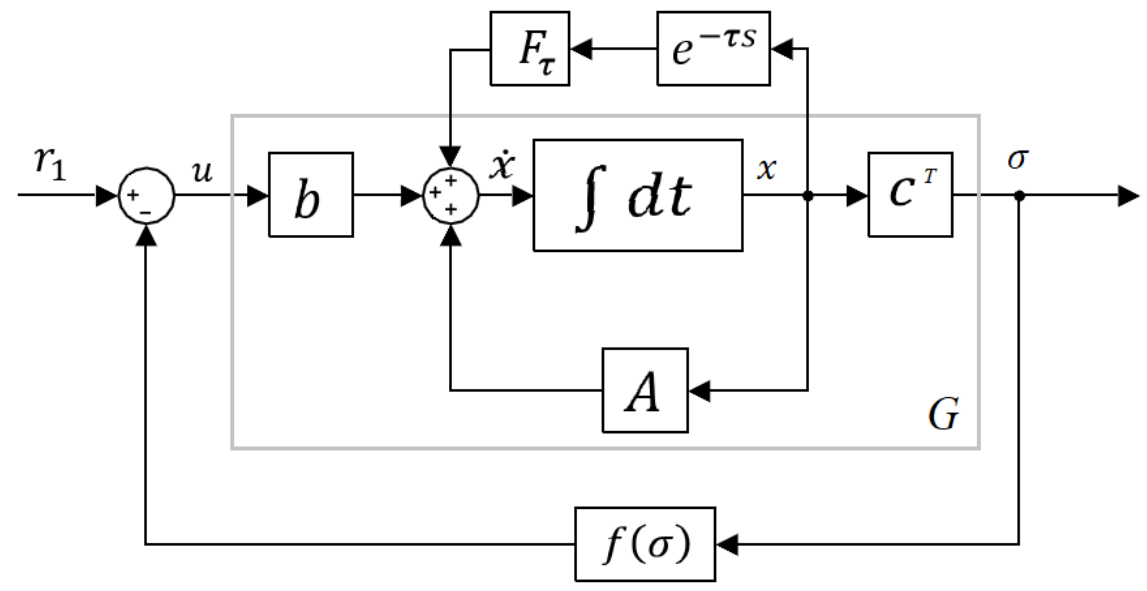

Source: Author. 
Figure 32: Simplified block diagram.

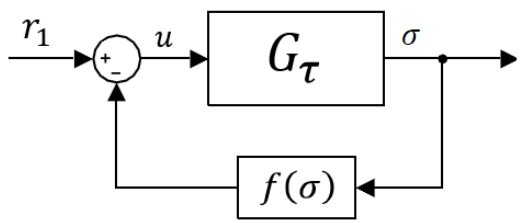

Source: Author.

The equation for system of Figure 31 , for $r_{1}=0$, is:

$$
\left\{\begin{array}{l}
\dot{x}=A x+b u+F_{\tau} x(t-\tau) \\
\sigma=c^{T} x
\end{array}\right.
$$

Theorem 15. Let be $f \in F_{\left[k_{1}, k_{2}\right]}$ and for a real fixed delay $\in[0, \tau]$ assume that the nominal system $G_{o}^{\tau}(s)$ in closed loop is stable. Then the system (3.9) is absolutely stable for some class of functions in the sector, if:

$$
\left|\frac{\left(\frac{k_{2}-k_{1}}{2}\right) G_{o}^{\tau}(j w)}{1+G_{o}^{\tau}(j w)}\right|<1, \quad \forall \omega, k,
$$

where:

$$
G_{o}^{\tau}(s)=\frac{G_{\tau}(s)}{1-G_{\tau}(s)+G_{\tau}(s)\left(\frac{k_{2}+k 1}{2}\right)},
$$

and

$$
G_{\tau}(s)=c^{T}\left(s I-A-F_{\tau}[L / L]_{k}\right)^{-1} b .
$$

Proof. In order to obtain $G_{\tau}(s)$, as shown in the Figure 32, one must obtain the transfer function that relates the input signal $u$ to the output signal $\sigma$ applying the Laplace transform to the equation (3.10):

$$
\begin{gathered}
s X(s)=A X(s)+b U(s)+F_{\tau} e^{-\tau s} \\
X(s)\left(s I-A-F_{\tau} e^{-\tau s}\right)=b U(s) \\
\frac{X(s)}{U(s)}=\left(s I-A-F_{\tau} e^{-\tau s}\right)^{-1} b .
\end{gathered}
$$

But,

$$
\sigma(s)=c^{T} X(s)
$$

so:

$$
\frac{\sigma(s)}{U(s)}=c^{T}\left(s I-A-F_{\tau} e^{-\tau s}\right)^{-1} b=G_{\tau}(s) .
$$

Since one replaces the exponential term with the Padé approximation (see Equation 
(2.96)), it is needed to check if the sequence $\left(s I-A-F_{\tau}[L / L]_{k}\right)^{-1}$ converge to $(s I-A-$ $\left.F_{\tau} e^{-\tau s}\right)^{-1}$. By the Lemmas 7 and 8 , one has which the sequence $\left(s I-A-F_{\tau}[L / M]_{k}\right)^{-1}$ converge point-to-point and uniformly (for more details see section 4.3). From this point on, the proof is analogous to the proof of the Theorem 14, replacing $G(s)$ with $G_{\tau}(s)$.

In the following, a comparison with the paper of (HE et al., 2005) is made, where it is presented a sufficient condition to absolute stability (see Theorem 7 in that paper).

Example 2. The system (3.13) presents a Chua's Circuit in state equations.

$$
\left\{\begin{array}{l}
\dot{x_{1}}(t)=\alpha\left[-x_{1}(t)+x_{2}-f\left(x_{1}(t)\right)\right]+F_{11} x(t-\tau)+F_{12} x(t-\tau)+F_{13} x(t-\tau), \\
\dot{x_{2}}(t)=x_{1}(t)-x_{2}(t)+x_{3}(t)+F_{21} x(t-\tau)+F_{22} x(t-\tau)+F_{23} x(t-\tau), \\
\dot{x_{3}}(t)=-\beta x_{2}(t)-\vartheta x_{3}(t)+F_{31} x(t-\tau)+F_{32} x(t-\tau)+F_{33} x(t-\tau),
\end{array}\right.
$$

where $x_{1}(t), x_{2}(t)$ and $x_{3}(t)$ are state variables. The nonlinearity, $f \in F_{\left[k_{1}, k_{2}\right]}$, is:

$$
f\left(x_{1}(t)\right)=m_{1} x_{1}(t)+\frac{1}{2}\left(m_{0}-m_{1}\right)\left(\left|x_{1}(t)+1\right|-\left|x_{1}(t)-1\right|\right),
$$

and $\alpha, \beta, \vartheta, m_{0}$ and $m_{1}$ are real numbers. Furthermore, $m_{0}=k_{2}$ and $m_{1}=k_{1}$ are the bounds of the sector. Placing in the form of (3.9), a matrix $F$ is inserted with the delay parameters ( $F$ is in Table 3 ) and the matrices $A, b$ and $c$ are:

$$
A=\left[\begin{array}{ccc}
-\alpha & \alpha & 0 \\
1 & -1 & 1 \\
0 & -\beta & \vartheta
\end{array}\right], \quad b=\left[\begin{array}{c}
-\alpha \\
0 \\
0
\end{array}\right], \quad c=\left[\begin{array}{l}
1 \\
0 \\
0
\end{array}\right]
$$

The example analyzes the influence of time-delay $\tau$ on the absolute stability of the Chua's circuit until a maximum value $\tau_{\max }$. In simulations, via Simulink ${ }^{\circledR}$, the parameters of the time-delay Chua's circuit are set as $\alpha=-1.3018, \beta=-0.0136, \vartheta=-0.0297, m_{0}=$ 0.1691, $m_{1}=-0.4768$. Simulating with $\tau=0.8$, the Figure 33 shows the Chua's circuit is oscillatory. Now, one applies the condition (3.11) of the Theorem 15. Performing the stability test via condition (3.11), according to Figure 34 it is predicted absolute stability for $\tau_{\max }=0.4206$. The $\tau_{\max }$ value was obtained with a few attempts in the stability test using the condition (3.11). 
Figure 33: Chua's Circuit unstable with $\tau=0.8$.

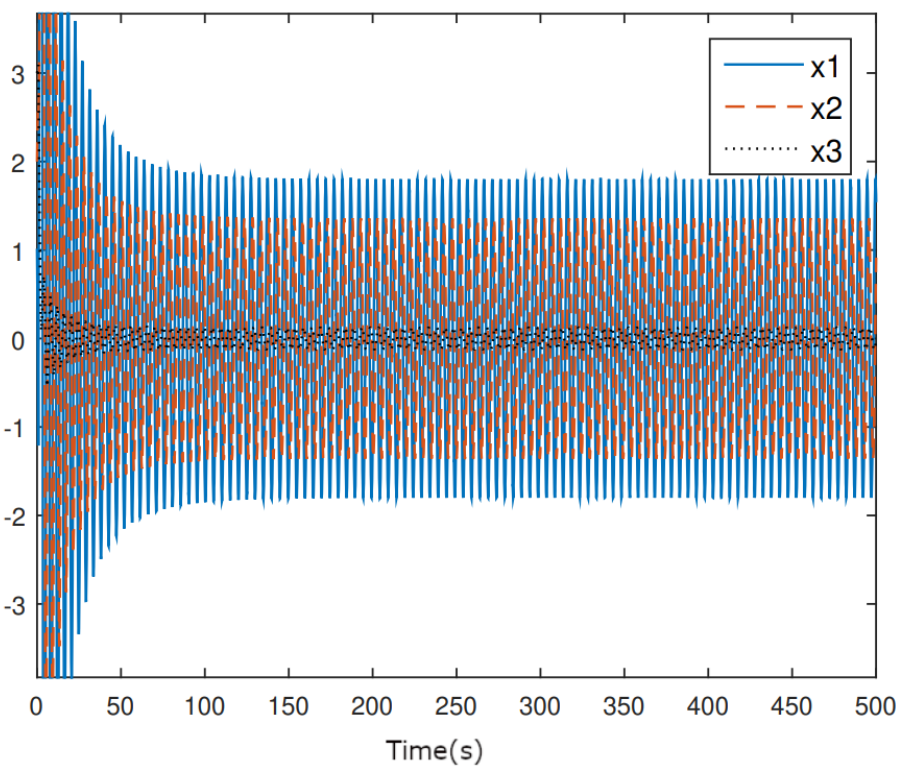

Source: Author.

Figure 34: Condition (3.11) satisfied for $\tau_{\max }=0.4206$ visualized via Bode diagram.

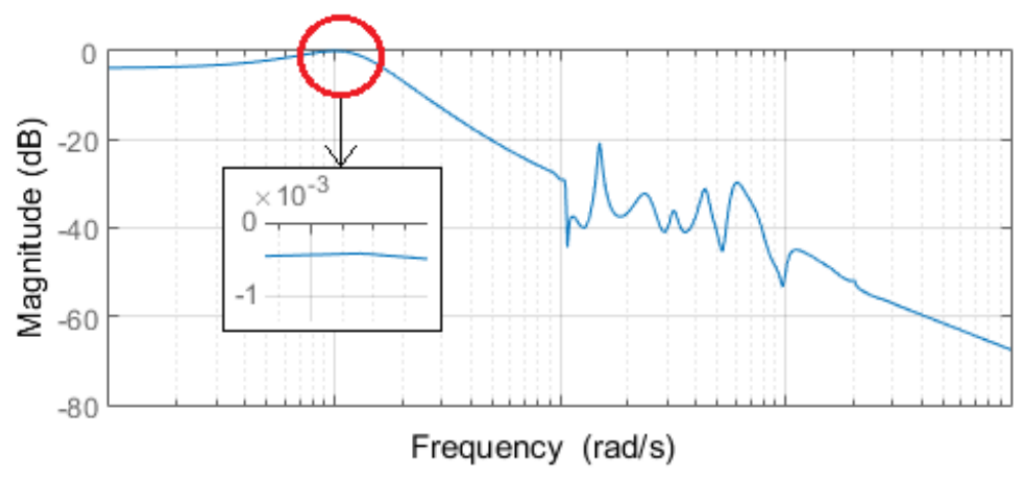

Source: Author.

The Table 3 shows a comparison between $\tau_{\text {max }}$ predicted by Theorem 7 of (HE et al., 2005) and the developed Theorem 15, showing that the criterion is less conservative. In Table 4, different values of $\tau_{\text {max }}$ for different orders of Padé approximation are shown for the developed criterion. It can be seen that the value of $\tau_{\max }$ converges to 0.4206 as $r \rightarrow \infty$. In time simulation via Simulink ${ }^{\circledR}$, the absolute stability is verified for $\tau=0.4206$ (see Figure 35). 
Table 3: $\tau_{\max }$

\begin{tabular}{|c|c|c|c|c|}
\hline $\mathrm{F}$ & & & Theorem 7 in (HE et al., 2005) & Theorem 15 \\
\hline-0.8 & 0.5 & 3.6 & \multirow{3}{*}{0.0284} & \multirow{3}{*}{0.4206} \\
\hline 0.4 & 0.1 & -0.1 & & \\
\hline 0.4 & 0.01 & -0.8 & & \\
\hline
\end{tabular}

Source: Author.

Table 4: $\tau_{\max }$ and Padé approximation order

\begin{tabular}{llllll}
\hline Order & 1 & 2 & 5 & 9 & 10 \\
\hline$\tau_{\max }$ & 0.4301 & 0.4207 & 0.4206 & 0.4206 & 0.4206 \\
\hline \multicolumn{5}{c}{ Source: Author. }
\end{tabular}

Figure 35: Absolute Stability to Chua's Circuit with $\tau=0.4206$ via Simulink ${ }^{\circledR}$.

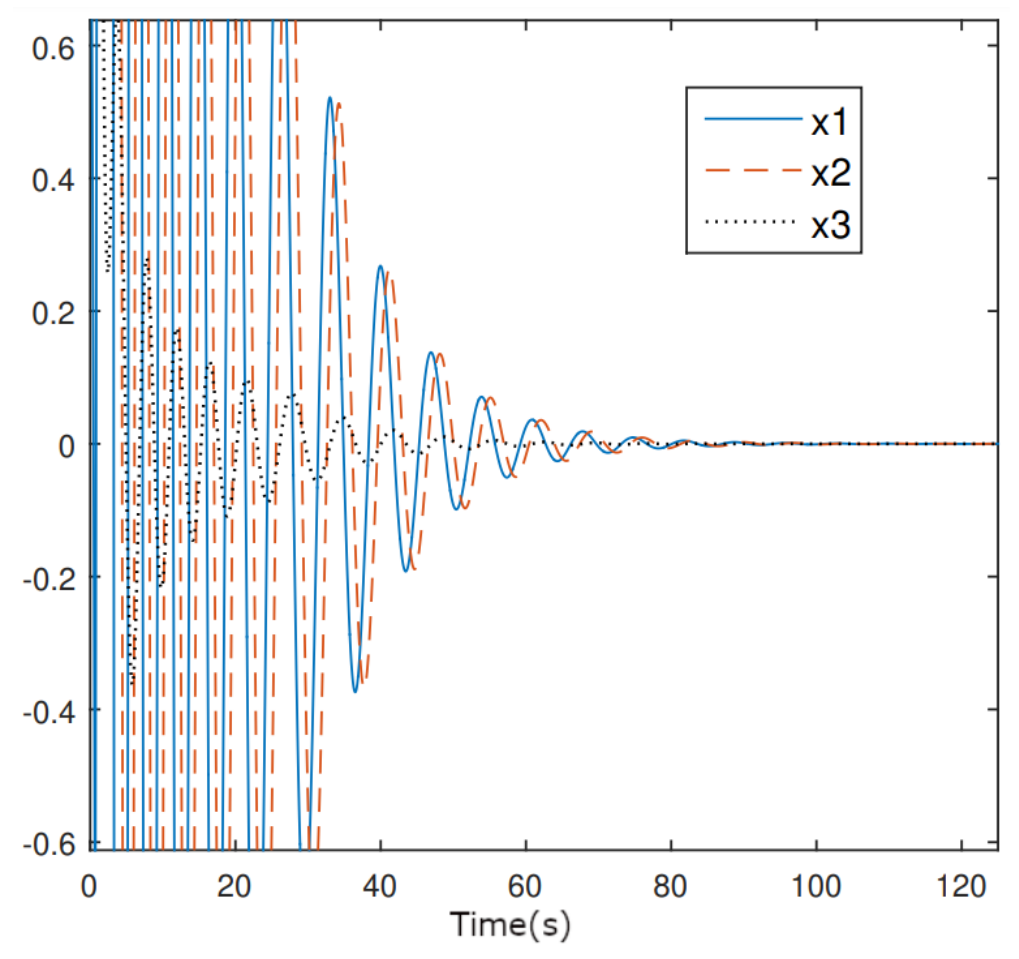

Source: Author.

\subsection{Synthesis of the Controller in Continuous Case}

Theorems 13 and 14 use the $K=1$, as the objective was to analyze the absolute stability of the Lurie type system. In the case of controller design $(K \neq 1)$, the idea is simpler, not being necessary to use the Lemma 3. 
Thus, to design a controller for the Lurie type system (1.1) stable and with good performance, one can use the control system of the Figure (19). Theorems 16 and 17 establish the technique of design of controllers for Lurie type systems for two classes of nonlinearities.

Theorem 16. Given a controller $K(s)$ and $[0, k]$ the sector, for some class of functions $f \in F_{(0, k]}$, the system (2.27) is robustly stable and have robustness of performance, if and only if:

$$
\left\|\begin{array}{c}
W_{p} S \\
W_{u} K(s) S \\
W_{I M} T
\end{array}\right\|_{\infty}<1
$$

where:

$$
\begin{gathered}
S=\left(1+G_{o} K(s)\right)^{-1}, \\
T=\left(1+G_{o} K(s)\right)^{-1} G_{o} K(s), \\
W_{I M}(s)=G_{o}(s) \frac{k}{2},
\end{gathered}
$$

with:

$$
G_{o}(s)=\frac{G(s)}{1+G(s) \frac{k}{2}} .
$$

$W_{p}$ and $W_{u}$ are chosen to match specification (see section 2.5).

Proof. First of all, the system (1.1) can be placed in form of Figure 17 and Figure 18, from the (2.27), one has:

$$
G_{p}(s)=\frac{G(s)}{1+\alpha G(s)},
$$

since one has $f \in F_{(0, k]}$ we do $\alpha=\frac{k}{2}+\frac{k}{2} \delta,|\delta| \leq 1$. So:

$$
G_{p}(s)=\frac{G(s)}{1+G(s) \frac{k}{2}+G(s) \frac{k}{2} \delta},
$$

dividing the numerator and denominator by $1+G(s) \frac{k}{2}$, one has:

$$
G_{p}(s)=\frac{\frac{G(s)}{1+G(s) \frac{k}{2}}}{1+\left(\frac{G(s) \frac{k}{2}}{1+G(s) \frac{k}{2}}\right) \delta},
$$

so one has inverse multiplicative uncertainty, according to $(2.32): G_{p}(s)=\frac{G_{o}(s)}{1+W_{I M}(s) \delta}, \quad|\delta| \leq$ 1. Where:

$$
G_{o}(s)=\frac{G(s)}{1+G(s) \frac{k}{2}},
$$




$$
W_{I M}(s)=G_{o}(s) \frac{k}{2}
$$

Now, the weights $W_{p}$ and $W_{u}$ must be inserted in order to obtain a system according to Figure 19. Finally, guaranteeing that (2.38) is satisfied, the theorem is demonstrated.

Theorem 17. Given a controller $K(s)$ and $\left[k_{1}, k_{2}\right]$ the sector, for some class of functions $f \in F_{\left[k_{1}, k_{2}\right]}$, then the system (2.27) is robustly stable and have robustness of performance, if and only if:

$$
\left\|\begin{array}{c}
W_{p} S \\
W_{u} K(s) S \\
W_{I M}^{k} T
\end{array}\right\|_{\infty}<1
$$

where:

$$
\begin{gathered}
S=\left(1+G_{o}^{k}(s) K(s)\right)^{-1}, \\
T=\left(1+G_{o}^{k}(s) K(s)\right)^{-1} G_{o}^{k}(s) K(s), \\
W_{I M}^{k}(s)=G_{o}^{k}(s)\left(\frac{k_{2}+k_{1}}{2}\right),
\end{gathered}
$$

with:

$$
G_{o}^{k}(s)=\frac{G(s)}{1+G(s)\left(\frac{k_{2}+k_{1}}{2}\right)} .
$$

$W_{p}$ and $W_{u}$ are chosen to match specification (see section 2.5).

Proof. The proof is similar to the previous theorem. In this case, one uses $k_{1} \leq \alpha \leq k_{2}$, thus:

$$
\alpha=k_{1}+\left(\frac{k_{2}-k_{1}}{2}\right)+\frac{\left(k_{2}-k_{1}\right)}{2} \delta=\left(\frac{k_{2}+k_{1}}{2}\right)+\frac{\left(k_{2}-k_{1}\right)}{2} \delta, \quad|\delta| \leq 1 .
$$

Following the steps of the previous theorem, the demonstration is completed without difficulty.

Example 3. Design a controller by $\mathcal{H}_{\infty}$ Mixed - Sensitivity $(S / K S / T)$ for the Lurie type system of Example 1.

In the example, the sector is $f \in F_{(0,1]}$, thus $k=1$ is adopted. So, by Theorem 16, one has:

$$
G_{o}(s)=\frac{G(s)}{1+\frac{G(s)}{2}},
$$


and

$$
W_{I M}=\frac{\frac{G(s)}{2}}{1+\frac{G(s)}{2}} .
$$

For $W_{p}(s)=\frac{s / M+w_{b}}{s+w_{b} E}$, the parameters $M=2, E=5 \cdot 10^{-5}$ and $w_{b}=10$ was used. It was chosen $E<<1$, to obtain a small stationary error. $M=2$ was chosen according to the value suggested in (SKOGESTAD; POSTLETHWAITE, 2007). It was chosen $w_{b}=10$ in order to have a larger bandwidth, rendering the system response faster. Finally, using the Matlab ${ }^{\circledR}$ function mixsyn $\left(G_{o}, W_{p}, W_{I M}, W_{u}\right)$ the controller is obtained. The $\max _{\omega} \bar{\sigma}(j \omega)=1.0065$ of (2.38) was reached with $W_{u}=0.8$. This value for $W_{u}$ was obtained in a few attempts.

A time simulation was performed using the nonlinear function $f(\sigma)=\tanh (\sigma)$. In Figure 36 the response to a sine (Reference) with amplitude one is presented for the closed loop with unitary controller case (Without Controller), with a controller based in LMI ((CHENG; SAVKIN, 2007)-Lemma 3.4) and with controller proposed in this work (Controller $S / K S / T)$. It can be seen that the reference is not followed properly in the first case neither with the controller (CHENG; SAVKIN, 2007) (the $\max _{\omega} \bar{\sigma}(j \omega)$ value obtained with this controller is 1.7532). The reader can see that the developed controller $(S / K S / T)$ follows the reference much better.

Figure 36: Response to sine wave.

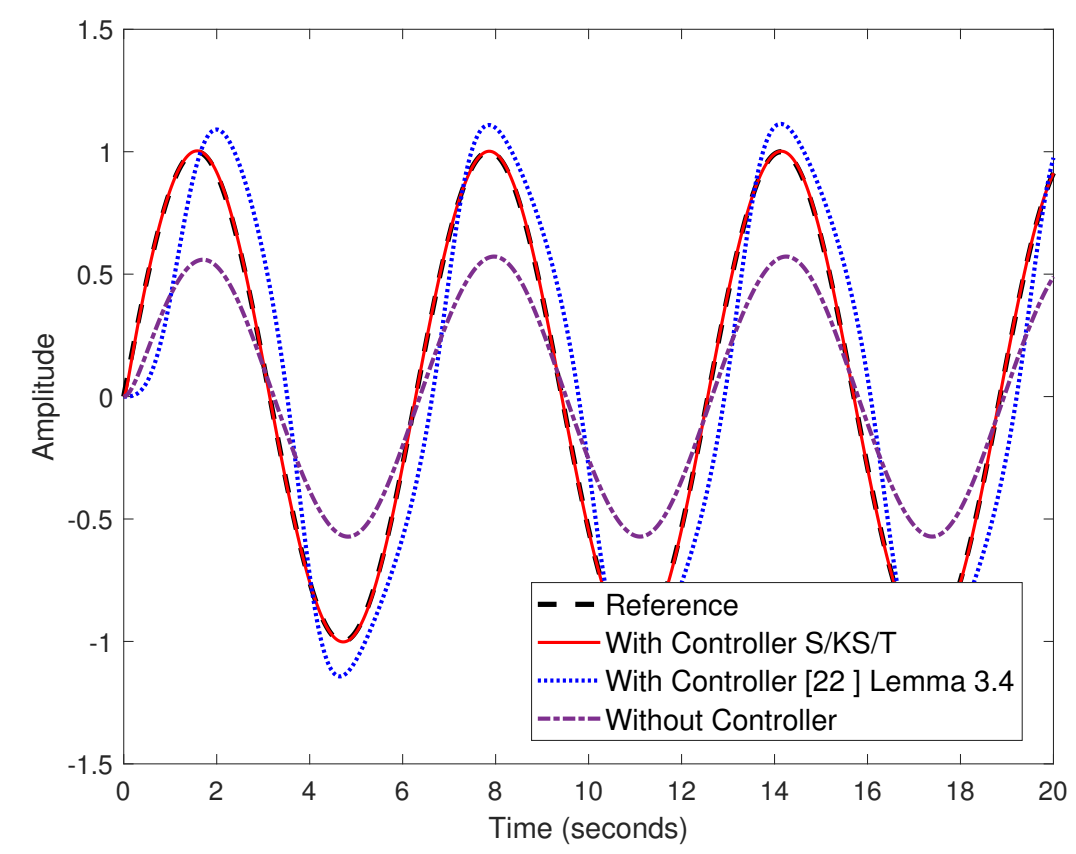

Source: Author.

A similar simulation was conducted for a unitary step reference signal, shown in 
Figure 3\%. Again, the reference is not properly followed with unitary controller or with controller of (CHENG; SAVKIN, 2007).

Figure 37: Response to step signal.

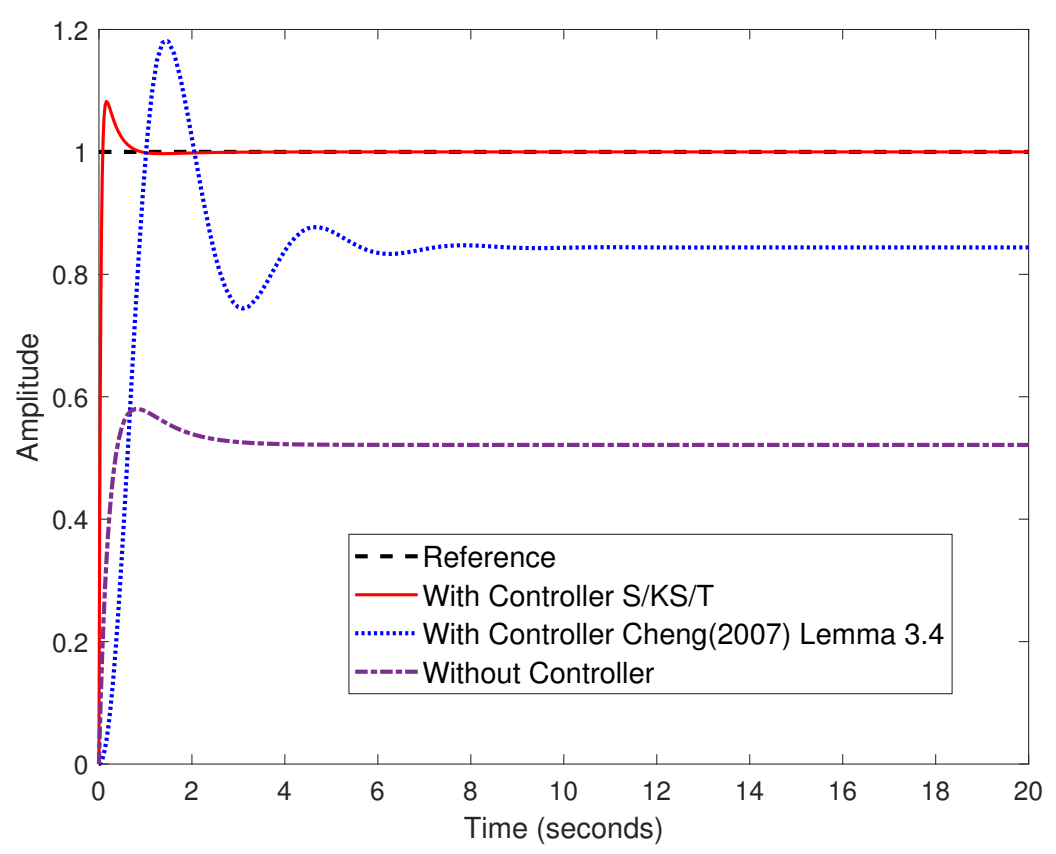

Source: Author.

\subsection{Approach in the Discrete Case}

The use of the synthesis technique for the discrete SISO case, via procedures according to sections 2.5.1 and 2.6.1, it is illustrated by means of the next example.

Figure 38: Block diagram of the numerical example.

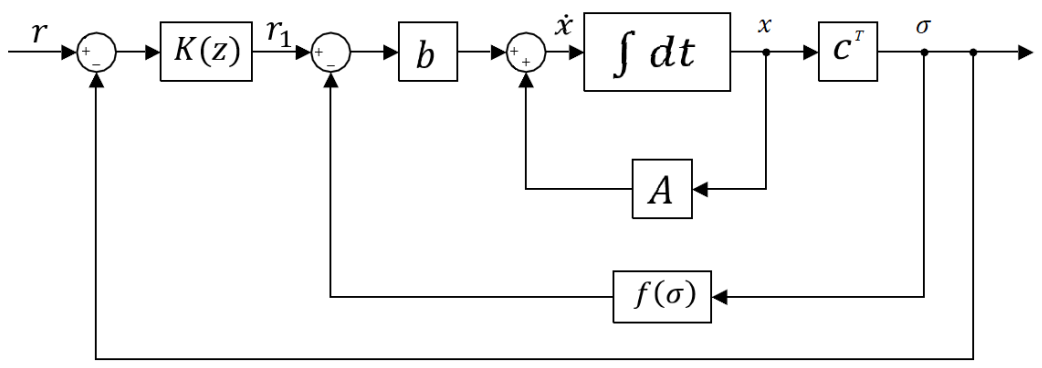

Source: Author.

Example 4. Design a discrete robust controller according to Figure 38, and also consider the Lurie system (3.16), $T_{s}=0.1$, and $k=2$ (in this case $f \in F_{(0,2]}$ ). 


$$
\left\{\begin{array}{l}
\dot{x}_{1}=-x_{1}+f(\sigma) \\
\dot{x}_{2}=-x_{2} \\
\sigma=x_{1}
\end{array}\right.
$$

Considering the matrices:

$$
\begin{gathered}
A=\left[\begin{array}{cc}
-1 & 0 \\
0 & -1
\end{array}\right], \quad b=\left[\begin{array}{l}
1 \\
0
\end{array}\right] \quad \text { and } \\
c^{T}=\left[\begin{array}{ll}
1 & 0
\end{array}\right],
\end{gathered}
$$

In order to proceed the design via Mixed-Sensitivity $\mathcal{H}_{\infty}(S / K S)$ and $(S / K S / T)$ with Parametric Uncertainties, first, one replaces $\alpha=\frac{k}{2}+\frac{k}{2} \delta,|\delta| \leq 1$. So, using (2.41) and (2.42) one gets the weight for uncertainty and the nominal plant $G(z)$, with this, one has: $G(z)=\frac{T s}{z-1+T s+\frac{k T s}{2}}$ and $W_{I}(z)=\frac{\frac{k T s}{2}}{z-1+T s+\frac{k T s}{2}}$.

\section{Controller design via $(S / K S)$ approach:}

In order to obtain $W_{p}$, it is used (2.37) and to its parameters is chosen as: $M=2$, $A=0.005$ e $w_{b}=10$ as suggested in (SKOGESTAD; POSTLETHWAITE, 2007), then $W_{p}(z)=\frac{0.5 z+0.4975}{z-0.995}$ is obtained. $W_{p}$ was discretized using the function $c 2 d$ of the Matlab ${ }^{\circledR}$. For the penalization of control, was chosen: $W_{u}=0.0561$. This value was obtained in a few attempts, until one gets the best fit. After obtaining the plant $P$ and using the Matlab ${ }^{\circledR}$ function hinfsyn, the following controller is obtained:

$$
K(z)=\frac{9.932 z^{4}-15.84 z^{3}-3.605 z^{2}+15.84 z-6.325}{z^{4}-1.792 z^{3}-0.2032 z^{2}+1.786 z-0.7907},
$$

with $\gamma$ of (2.43) equal to 1.0046.

Time Simulation $(S / K S)$ :

The first simulation is step response for some plants in the falmily of parameters $0<\alpha \leq 2$ of $G_{p}$ randomly chosen. It is possible to observe, in Figure 39, that the controller behave similarly for all the plants tested. Next, simulations via Simulink ${ }^{\circledR}$ with nonlinearity equal to a hyperbolic tangent was performed, as shown in Figure 40. Herewith, it was obtained responses to sine and step wave of amplitude equal to 5, as shown in Figure 41 and Figure 42. 
Figure 39: Step response of the family of plants with controller.

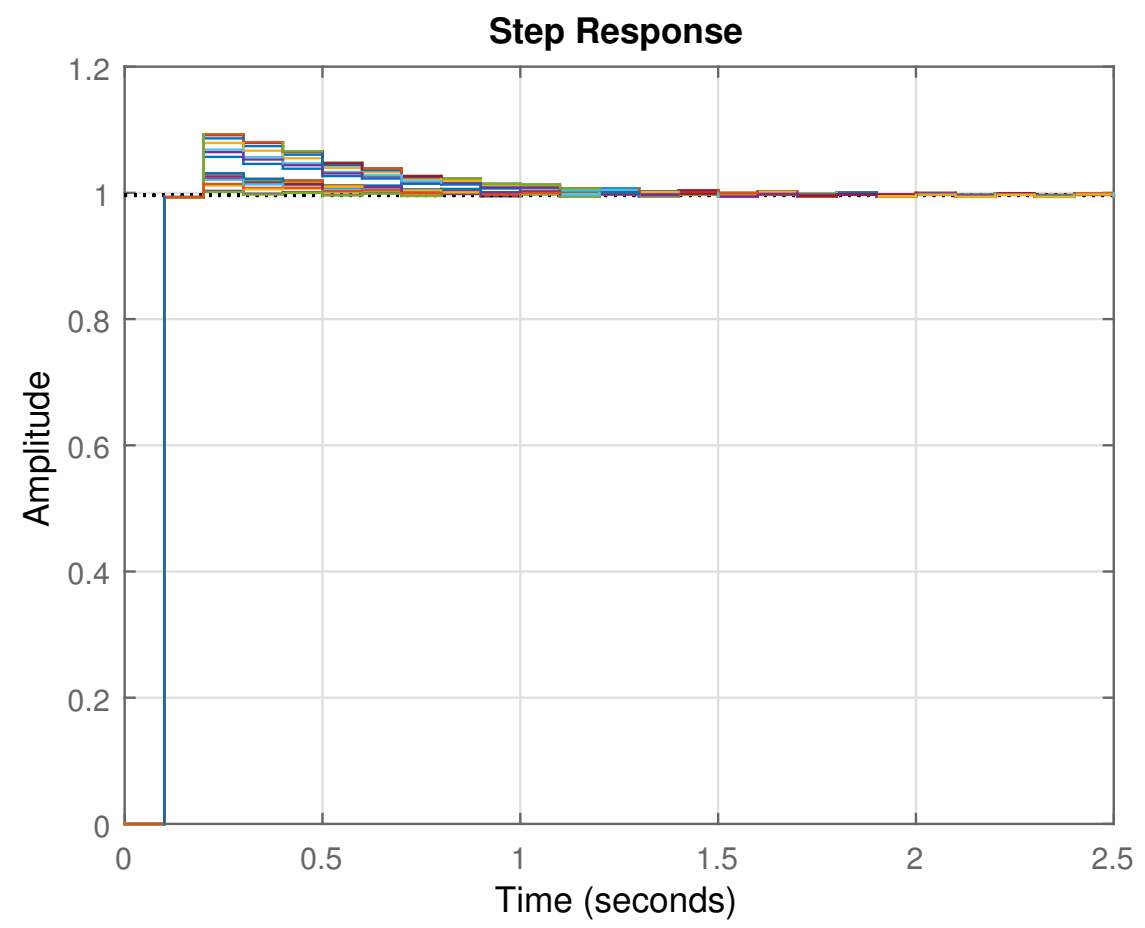

Source: Author.

Figure 40: The hyperbolic tangent.

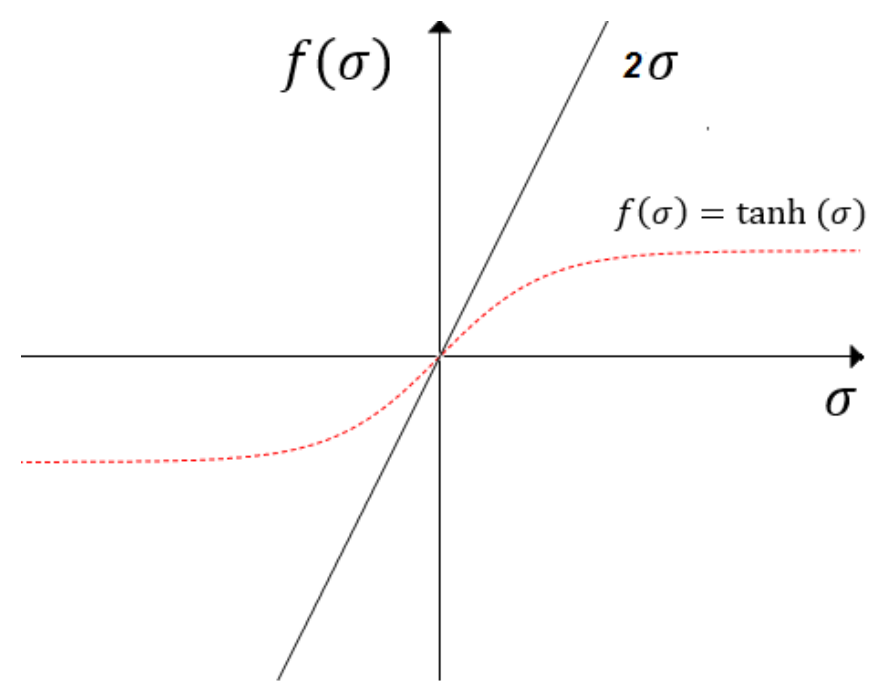

Source: Author. 
Figure 41: Response to sine wave with discrete controller by mixed-sensitivity $(S / K S)$ with continuous plants.

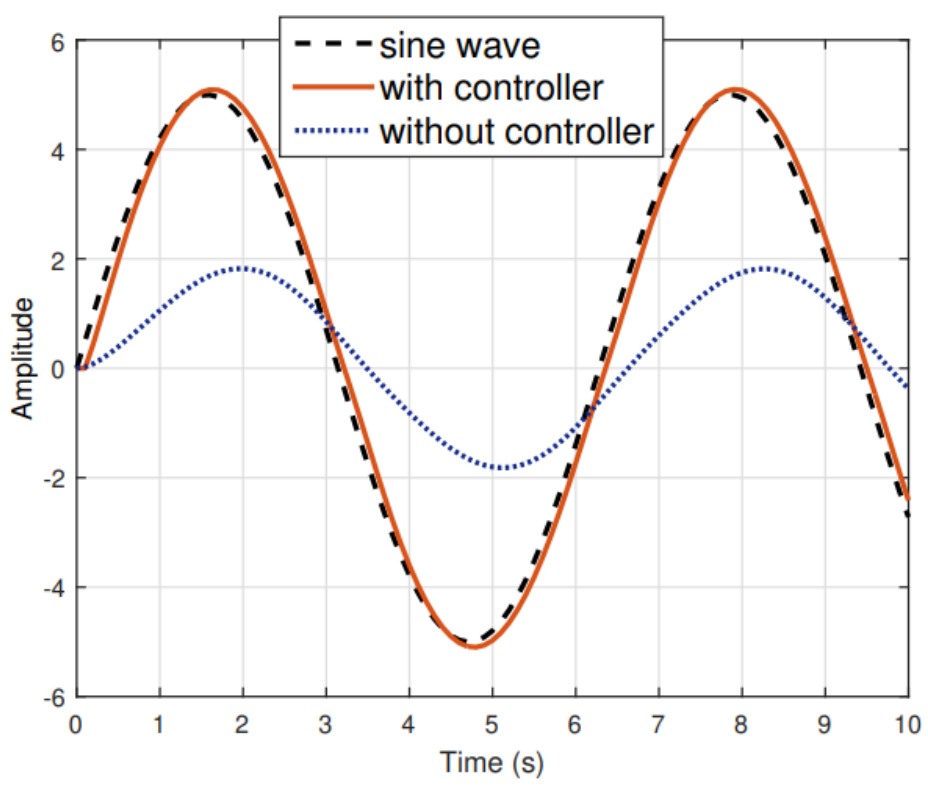

Source: Author.

Figure 42: Response to pulse wave with discrete controller by mixed-sensitivity $(S / K S)$ with continuous plants.

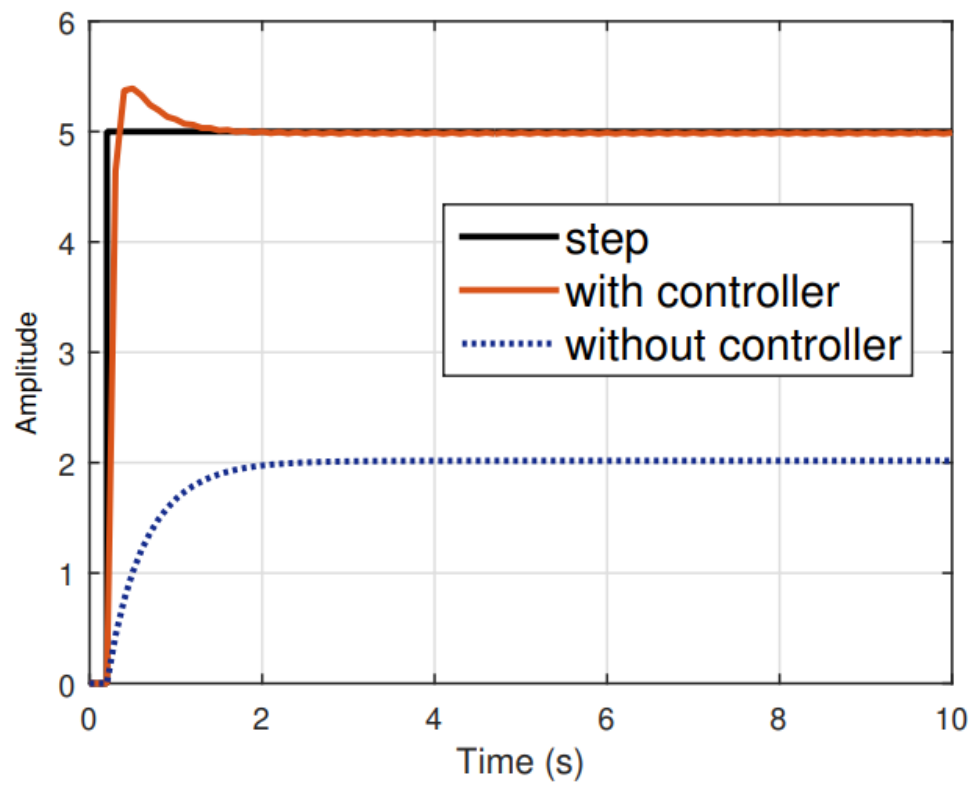

Source: Author.

Observe that the controller behave as expected, following the reference signal. Even with the presence of hyperbolic tangent as nonlinearity, the controller still follows the reference in both simulations. 


\section{Controller Obtained $(S / K S / T)$ :}

In order to obtain $W_{p}$, it was used (2.37) and for its parameters it was made a little different that suggested in (SKOGESTAD; POSTLETHWAITE, 2007), it was chosen $M=5, A=0.005$ and $w_{b}=1 \mathrm{rad} / \mathrm{s}$, with a fine adjustment of these parameters one gets a good value for the norm. One discretizes $W_{p}$ using the function $c 2 d$ of Matlab ${ }^{\circledR}$, getting: $W_{p}(z)=\frac{0.2 z-0.1}{z-0.9995}$. For the penalization of control, it was chosen $W_{u}=0.01$, this value was also obtained in a few attempts. Using the Matlab ${ }^{\circledR}$ function mixsyn one obtains the controller:

$$
K(z)=\frac{7.497 z^{3}-4.397 z^{2}-7.358 z+4.862}{z^{3}-1.024 z^{2}-0.5958 z+0.6201}
$$

with value of norm of (2.43): $\gamma=1.0064$.

\section{Time Simulation $(S / K S / T)$ :}

Analogously to the $(S / K S)$ design, one checks the robustness of the controller. One has in the Figure 43 the response to the unitary step, for not only the nominal plant $G$, but for other plants randomly chosen according to the uncertainty parameter $0<\alpha \leq 2$ of $G_{p}$.

Figure 43: Step response of the family of plants with controller $(\mathrm{S} / \mathrm{KS} / \mathrm{T})$.

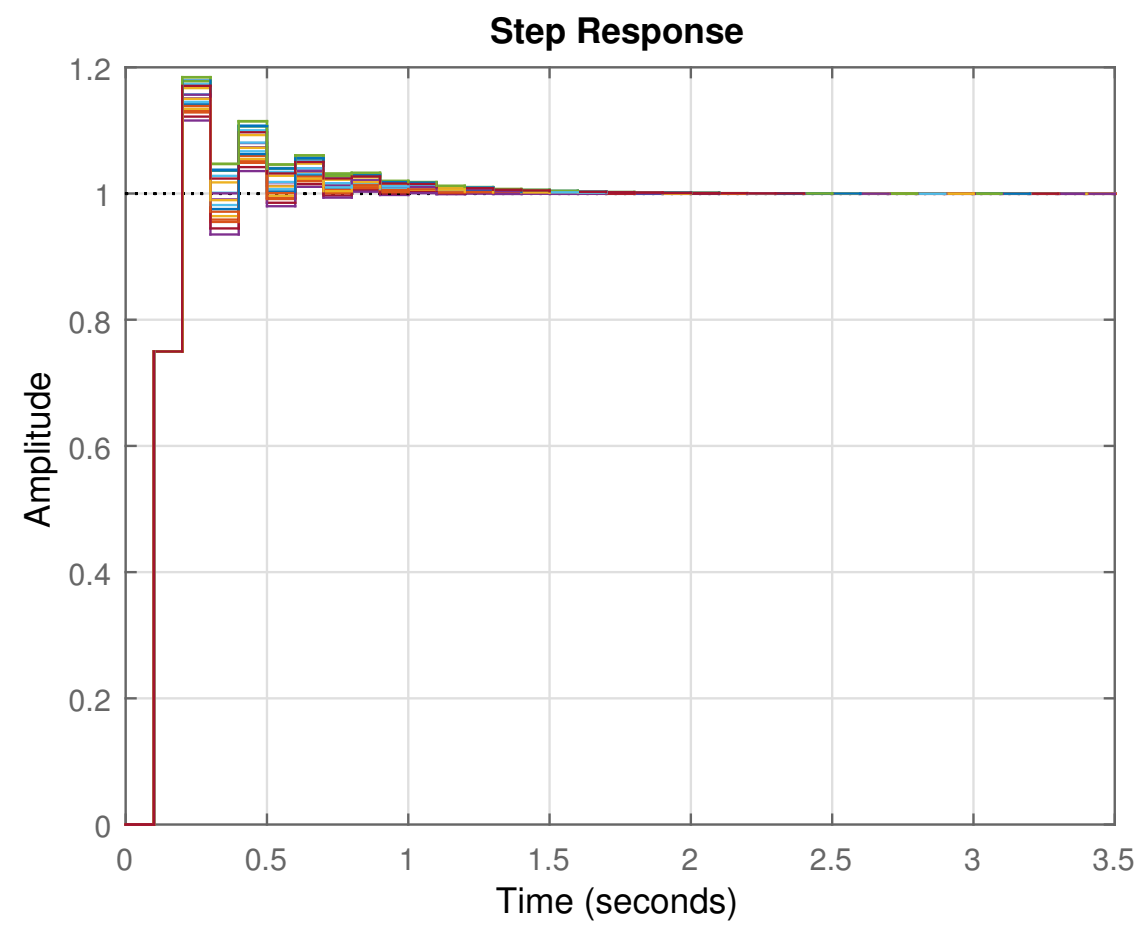

Source: Author.

Similarly to the previous simulation, the controller works as intended for all the plants 
tested. In simulations via Simulink ${ }^{\circledR}$ with nonlinearity equal to the hyperbolic tangent, one has Figure 44 and Figure 45 responses to sine and step inputs with amplitude 5.

Figure 44: Response to sine wave with discrete controller by mixed-sensitivity $(S / K S / T)$ with continuous plants.

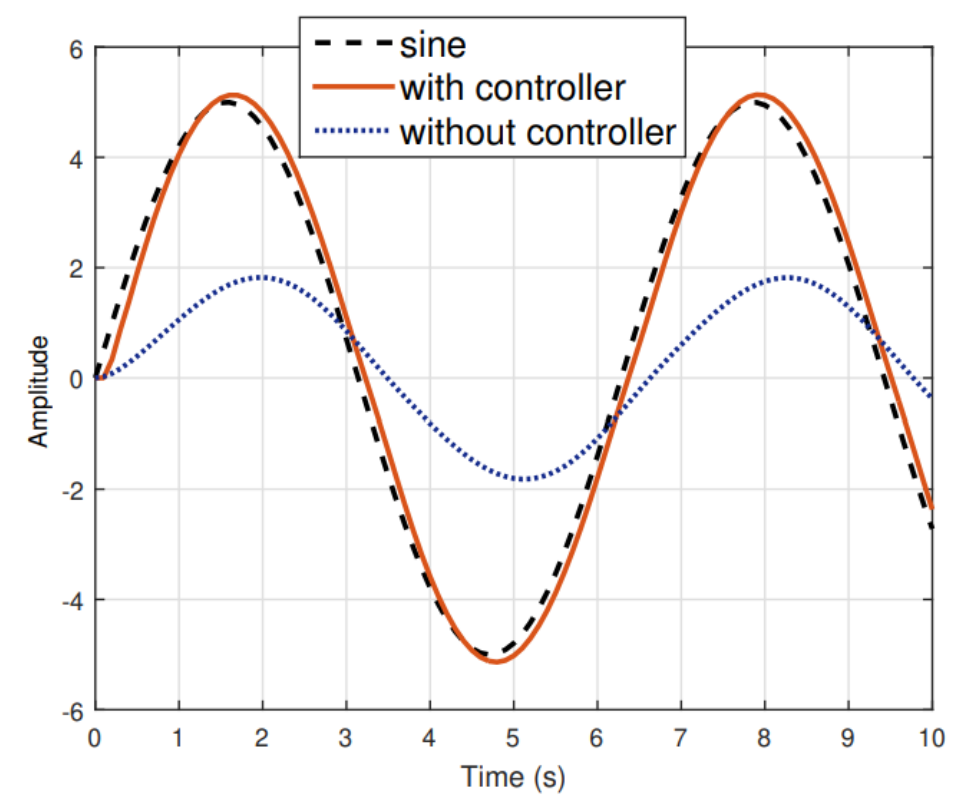

Source: Author.

Figure 45: Response to pulse wave with discrete controller by mixed-sensitivity $(S / K S / T)$ with continuous plants.

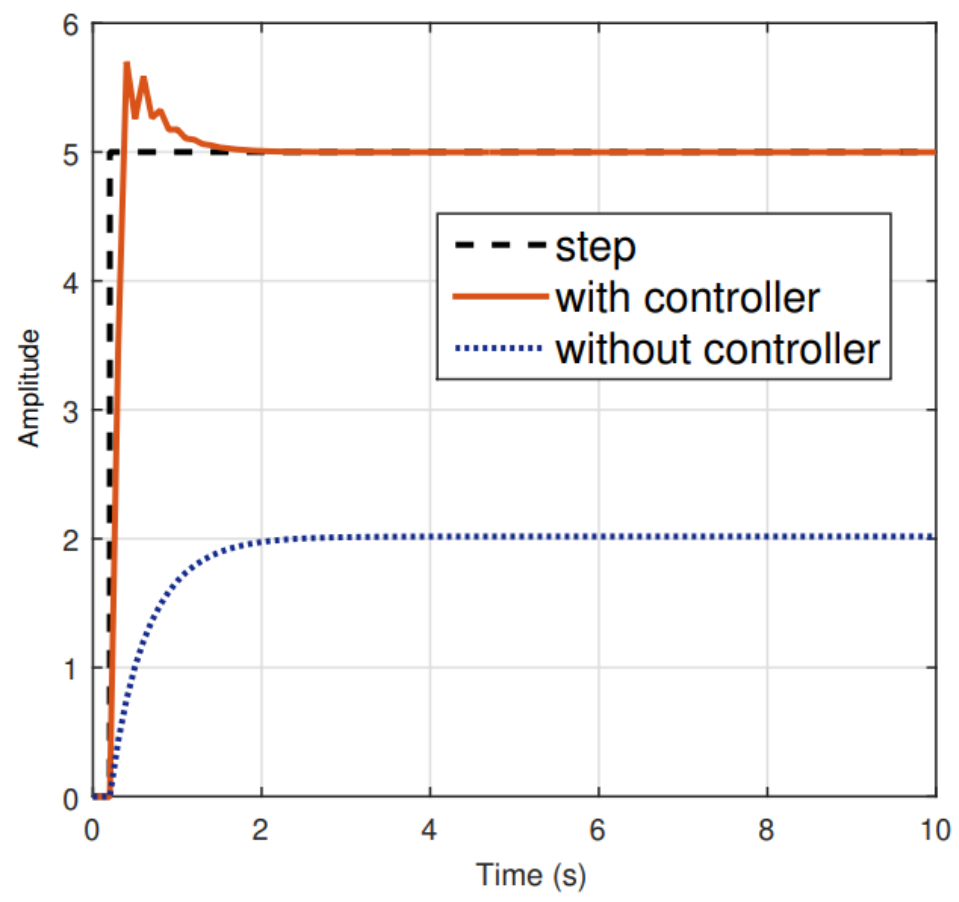

Source: Author. 


\section{Controllers obtained using LMI and Polytopic Uncertainties:}

The controller is obtained using the LMI (2.51) of the Theorem 5. The Table 5 presents the controller and also the $\xi$ values obtained, where $l$ is the number of constraints on the $L M I$.

Table 5: Controller obtained using Theorem 5 for $l \in[2,3,4]$.

\begin{tabular}{|l|c|c|}
\hline & $\|H\|_{\infty}$ Controller via LMI & $\xi$ value \\
\hline$l=2$ & {$[-16.4241]$} & 1.4805 \\
\hline$l=3$ & {$[-24.6527]$} & 1.0059 \\
\hline$l=4$ & {$[-29.4739]$} & 1.2421 \\
\hline
\end{tabular}

Source: Author.

\section{Temporal Simulation:}

We used in the simulations the nonlinearity $f(\sigma)=\tanh (\sigma)$, as shown in Figure 40. Differently from the two previous cases, as shown in Figure 46, the controller using the LMI approach presented a higher error, on the other hand, there is no overshooting.

Figure 46: Graphics obtained using all the controllers designed via LMI and polytopic uncertainties, and without controller (w/o).

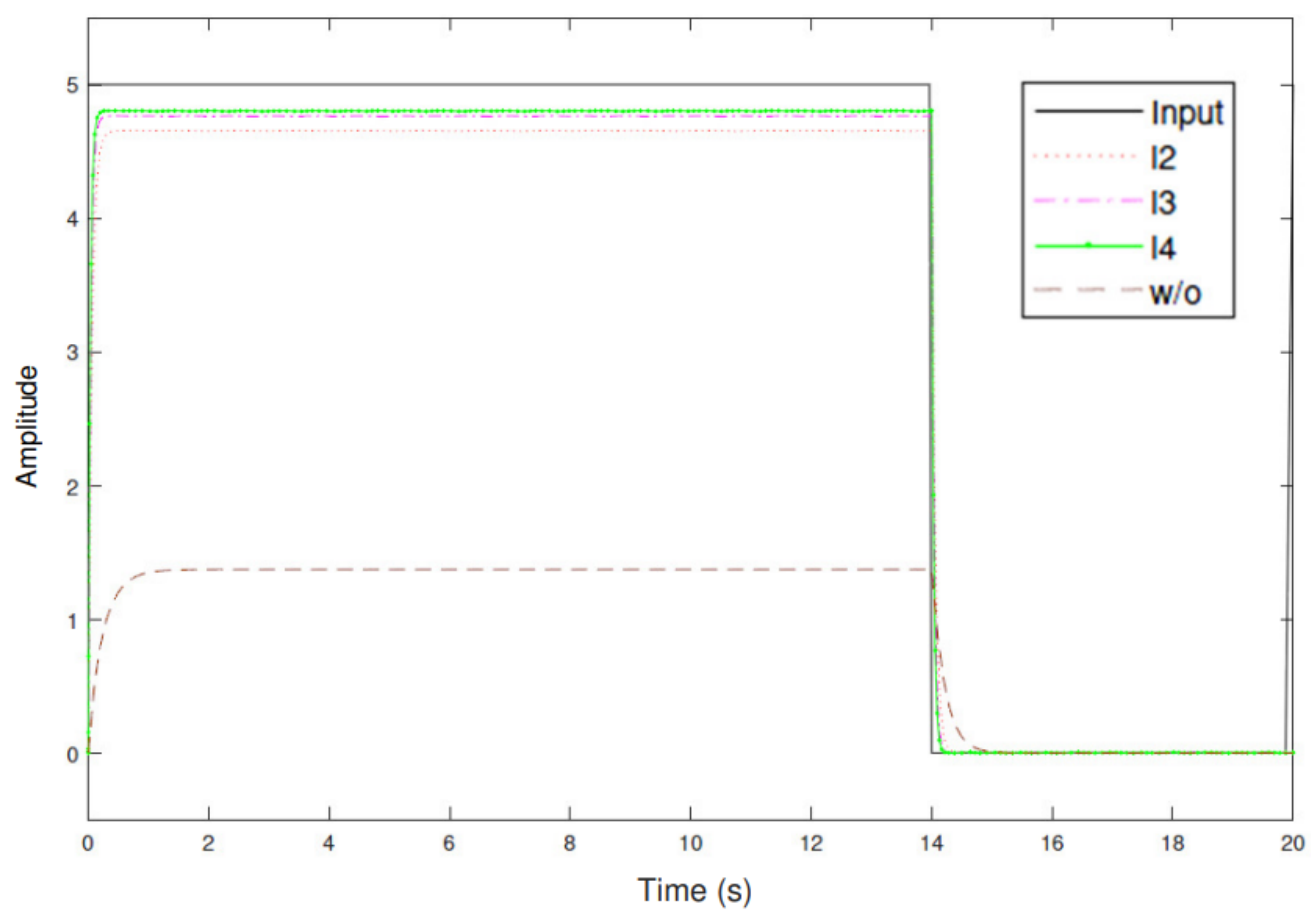

Source: Author. 


\section{SUFFICIENT CONDITIONS IN MIMO CASE}

The results for MIMO Lurie type systems are divided into three parts. Firstly, one comes up with results that bring forward two sufficient condition for the original LP of the Figure 6 using the Theorem 7 of the section 2.7.2. Secondly, a new condition is presented for time-delay system and applied in Chua's circuits. Chua's circuit (MATSUMOTO, 1984 ) is an electronic circuit that exhibits classic chaotic behavior. Thirdly, one extends the result to the closed-loop LP (Figure 21) and finally, one proposes the synthesis of a controller via DK-iteration for Lurie type systems with multiple inputs.

\subsection{Sufficient Conditions}

The next lemma, proved by the authors, puts the system (2.28) according to Figure 15.

Lemma 4. The system (2.28) with $f_{j} \in F_{\left(0, k_{j}\right]}$, has a $\Delta$-Structure given by $\Delta=\left\{\right.$ diag $\left[\delta_{1}\right.$, ..., $\left.\left.\delta_{m}\right]:\left|\delta_{j}\right| \leq 1\right\}$, with:

$$
\Lambda=\left[\begin{array}{ccccc}
A_{0} & B_{0} & E_{1} & \ldots & E_{m} \\
C_{0} & D_{0} & F_{1} & \ldots & F_{m} \\
G_{1} & H_{1} & 0 & \ldots & 0 \\
\vdots & \vdots & \vdots & \ddots & \vdots \\
G_{m} & H_{m} & 0 & \ldots & 0
\end{array}\right]
$$

where $\Lambda$ is the matrix of section 2.3.1, and:

$$
A_{0}=\left[\begin{array}{ccc}
a_{11}-\sum_{j=1}^{m} b_{1 j} \frac{k_{j}}{2} c_{j 1} & \ldots & a_{1 n}-\sum_{j=1}^{m} b_{1 j} \frac{k_{j}}{2} c_{j n} \\
\vdots & \ddots & \vdots \\
a_{n 1}-\sum_{j=1}^{m} b_{n j} \frac{k_{j}}{2} c_{j 1} & \ldots & a_{n n}-\sum_{j=1}^{m} b_{n j} \frac{k_{j}}{2} c_{j n}
\end{array}\right]
$$




$$
\begin{gathered}
B_{0}=B, C_{0}=C, D_{0}=0, E_{j}=\left[\begin{array}{c}
-\frac{k_{j}}{2} b_{1 j} \\
\vdots \\
-\frac{k_{j}}{2} b_{n j}
\end{array}\right], F_{j}=0, G_{j}=\left[\begin{array}{l}
c_{j 1} \\
\vdots \\
c_{j n}
\end{array}\right]^{T}, H_{j}=0, \\
\text { for } \quad j=1, \ldots, m .
\end{gathered}
$$

Proof. In matrix form, the system (1.2) is:

$$
\begin{gathered}
{\left[\begin{array}{l}
\dot{x}_{1} \\
\vdots \\
\dot{x}_{n}
\end{array}\right]=\left[\begin{array}{ccc}
a_{11} & \ldots & a_{1 n} \\
\vdots & \ddots & \vdots \\
a_{n 1} & \ldots & a_{n n}
\end{array}\right]\left[\begin{array}{l}
x_{1} \\
\vdots \\
x_{n}
\end{array}\right]-\left[\begin{array}{ccc}
b_{11} & \ldots & b_{1 m} \\
\vdots & \ddots & \vdots \\
b_{n 1} & \ldots & b_{n m}
\end{array}\right]\left[\begin{array}{c}
f_{1}\left(\sigma_{1}\right) \\
\vdots \\
f_{m}\left(\sigma_{m}\right)
\end{array}\right]+B r_{1},} \\
{\left[\begin{array}{l}
\sigma_{1} \\
\vdots \\
\sigma_{m}
\end{array}\right]=\left[\begin{array}{ccc}
c_{11} & \ldots & c_{1 n} \\
\vdots & \ddots & \vdots \\
c_{m 1} & \ldots & c_{m n}
\end{array}\right]\left[\begin{array}{l}
x_{1} \\
\vdots \\
x_{n}
\end{array}\right]}
\end{gathered}
$$

Replacing $f_{j}\left(\sigma_{j}\right)$ for $\alpha_{j} \sigma_{j}, j=1, \ldots, m$, one has:

$$
\left[\begin{array}{c}
\dot{x}_{1} \\
\vdots \\
\dot{x}_{n}
\end{array}\right]=\left[\begin{array}{c}
a_{11} x_{1}+\ldots+a_{1 n} x_{n}-\left(b_{11} \alpha_{1} \sigma_{1}+\ldots+b_{1 m} \alpha_{m} \sigma_{m}\right) \\
\vdots \\
a_{n 1} x_{1}+\ldots+a_{n n} x_{n}-\left(b_{n 1} \alpha_{1} \sigma_{1}+\ldots+b_{n m} \alpha_{m} \sigma_{m}\right)
\end{array}\right]+B r_{1}
$$

Now, substituting $\sigma_{j}, j=1, \ldots, m$ for $\sum_{i=1}^{n} c_{j i} x_{i}$ and isolating $x$, one has:

$$
\left[\begin{array}{c}
\dot{x}_{1} \\
\vdots \\
\dot{x}_{n}
\end{array}\right]=\left[\begin{array}{ccc}
a_{11}-\left(b_{11} \alpha_{1} c_{11}\right. & \ldots & a_{1 n}-\left(b_{11} \alpha_{1} c_{1 n}\right. \\
\left.+\ldots+b_{1 m} \alpha_{m} c_{m 1}\right) & & \left.+\ldots+b_{1 m} \alpha_{m} c_{m n}\right) \\
\vdots & \ddots & \vdots \\
a_{n 1}-\left(b_{n 1} \alpha_{1} c_{11}\right. & \ldots & a_{n n}-\left(b_{n 1} \alpha_{1} c_{1 n}\right. \\
\left.+\ldots+b_{n m} \alpha_{m} c_{m 1}\right) & & \left.+\ldots+b_{n m} \alpha_{m} c_{m n}\right)
\end{array}\right]\left[\begin{array}{c}
x_{1} \\
\\
\vdots \\
x_{n}
\end{array}\right]+B r_{1}
$$

Note that one gets the form of system (2.28), that is, $\left\{\begin{array}{l}\dot{x}=A_{\alpha} x+B r_{1} \\ \sigma=C x\end{array}\right.$, where: 


$$
A_{\alpha}=\left[\begin{array}{ccc}
a_{11}-\left(b_{11} \alpha_{1} c_{11}\right. & \ldots & a_{1 n}-\left(b_{11} \alpha_{1} c_{1 n}\right. \\
\left.+\ldots+b_{1 m} \alpha_{m} c_{m 1}\right) & & \left.+\ldots+b_{1 m} \alpha_{m} c_{m n}\right) \\
\vdots & \ddots & \vdots \\
a_{n 1}-\left(b_{n 1} \alpha_{1} c_{11}\right. & \ldots & a_{n n}-\left(b_{n 1} \alpha_{1} c_{1 n}\right. \\
\left.+\ldots+b_{n m} \alpha_{m} c_{m 1}\right) & & \left.+\ldots+b_{n m} \alpha_{m} c_{m n}\right)
\end{array}\right]
$$

Applying the normalization, one has $\alpha_{j}=\frac{k_{j}}{2}+\frac{k_{j}}{2} \delta_{j},\left|\delta_{j}\right| \leq 1, j=1, \ldots, m$ :

$$
\begin{aligned}
& {\left[\begin{array}{c}
\dot{x}_{1} \\
\vdots \\
\dot{x}_{n}
\end{array}\right]=\left(\left[\begin{array}{ccc}
a_{11}-\left(b_{11} \frac{k_{1}}{2} c_{11}\right. & \ldots & a_{1 n}-\left(b_{11} \frac{k_{1}}{2} c_{1 n}\right. \\
\left.+\ldots+b_{1 m} \frac{k_{m}}{2} c_{m 1}\right) & & \left.+\ldots+b_{1 m} \frac{k_{m}}{2} c_{m n}\right) \\
\vdots & \ddots & \vdots \\
a_{n 1}-\left(b_{n 1} \frac{k_{1}}{2} c_{11}\right. & \ldots & a_{n n}-\left(b_{n 1} \frac{k_{1}}{2} c_{1 n}\right. \\
\left.+\ldots+b_{n m} \frac{k_{m}}{2} c_{m 1}\right) & & \left.+\ldots+b_{n m} \frac{k_{m}}{2} c_{m n}\right)
\end{array}\right]\right.} \\
& \left.-\left[\begin{array}{ccc}
\delta_{1} b_{11} \frac{k_{1}}{2} c_{11} & \ldots & \delta_{1} b_{11} \frac{k_{1}}{2} c_{1 n} \\
+\ldots+\delta_{m} b_{1 m} \frac{k_{m}}{2} c_{m 1} & & +\ldots+\delta_{m} b_{1 m} \frac{k_{m}}{2} c_{m n} \\
\vdots & \ddots & \vdots \\
\delta_{1} b_{n 1} \frac{k_{1}}{2} c_{11} & \ldots & \delta_{1} b_{n 1} \frac{k_{1}}{2} c_{1 n} \\
+\ldots+\delta_{m} b_{n m} \frac{k_{m}}{2} c_{m 1} & & +\ldots+\delta_{m} b_{n m} \frac{k_{m}}{2} c_{m n}
\end{array}\right]\right)\left[\begin{array}{l}
x_{1} \\
\\
\vdots \\
\\
x_{n}
\end{array}\right]+B r_{1}
\end{aligned}
$$

Thereby, one has the system in the form of (2.19), with $B_{0}=B, C_{0}=C, D_{0}=D_{j}=$ $C_{j}=B_{j}=0$, and:

$$
\dot{x}=\left(A_{0}+\sum_{j=1}^{m} \delta_{j} A_{j}\right) x+B r_{1}
$$

where

$$
A_{0}=\left[\begin{array}{ccc}
a_{11}-\left(b_{11} \frac{k_{1}}{2} c_{11}\right. & \ldots & a_{1 n}-\left(b_{11} \frac{k_{1}}{2} c_{1 n}\right. \\
\left.+\ldots+b_{1 m} \frac{k_{m}}{2} c_{m 1}\right) & & \left.+\ldots+b_{1 m} \frac{k_{m}}{2} c_{m n}\right) \\
\vdots & \ddots & \vdots \\
a_{n 1}-\left(b_{n 1} \frac{k_{1}}{2} c_{11}\right. & \ldots & a_{n n}-\left(b_{n 1} \frac{k_{1}}{2} c_{1 n}\right. \\
\left.+\ldots+b_{n m} \frac{k_{m}}{2} c_{m 1}\right) & & \left.+\ldots+b_{n m} \frac{k_{m}}{2} c_{m n}\right)
\end{array}\right]
$$


and

$$
A_{j}=\left[\begin{array}{ccc}
-b_{1 j} \frac{k_{j}}{2} c_{j 1} & \ldots & -b_{1 j} \frac{k_{j}}{2} c_{j n} \\
\vdots & \ddots & \vdots \\
-b_{n j} \frac{k_{j}}{2} c_{j 1} & \ldots & -b_{n j} \frac{k_{j}}{2} c_{j n}
\end{array}\right], \quad j=1, \ldots m
$$

But note that:

$$
A_{j}=\left[\begin{array}{c}
-\frac{k_{j}}{2} b_{1 j} \\
\vdots \\
-\frac{k_{j}}{2} b_{n j}
\end{array}\right]\left[\begin{array}{lll}
c_{j 1} & \ldots & c_{j n}
\end{array}\right]
$$

which means that $\operatorname{rank}\left[A_{j}\right]=1$, then $\rho_{j}=1$ (see $\left.(2.21)\right)$.

Thus, calculating (2.22), one has:

$$
\left[\begin{array}{cc}
A_{j} & B_{j} \\
C_{j} & D_{j}
\end{array}\right]=\left[\begin{array}{c}
E_{j} \\
F_{j}
\end{array}\right]\left[\begin{array}{ll}
G_{j} & H_{j}
\end{array}\right]=\left[\begin{array}{cc}
E_{j} G_{j} & E_{j} H_{j} \\
F_{j} G_{j} & F_{j} H_{j}
\end{array}\right]
$$

So:

$$
A_{j}=\left[\begin{array}{c}
-\frac{k_{j}}{2} b_{1 j} \\
\vdots \\
-\frac{k_{j}}{2} b_{n j}
\end{array}\right]\left[\begin{array}{lll}
c_{j 1} & \ldots & c_{j n}
\end{array}\right]=E_{j} G_{j} .
$$

For convenience, because it is immediate, one can choose:

$$
E_{j}=\left[\begin{array}{c}
-\frac{k_{j}}{2} b_{1 j} \\
\vdots \\
-\frac{k_{j}}{2} b_{n j}
\end{array}\right] \quad \text { and } \quad G_{j}=\left[\begin{array}{lll}
c_{j 1} & \ldots & c_{j n}
\end{array}\right]
$$

Consequently, $F_{j}=H_{j}=0$, which concludes the proof.

The previous lemma rearranged the system (2.28) according to Figure 15, which gives the possibility of constructing the next theorem. This theorem provides a sufficient condition for absolute stability of the system (1.2).

Theorem 18. Let $\left[0, k_{j}\right]$ be the sectors that limit $f_{j}\left(\sigma_{j}\right)$ such that $f_{j} \in F_{\left(0, k_{j}\right]}$ for $j=$ $1, \ldots, m$, and assume that $A_{0}$ is Hurwitz. Then, the system (1.2) is absolutely stable for some class of functions in the sector, if:

$$
\mu\left(\Lambda_{22}(j \omega)\right)<1, \quad \forall \omega
$$

where:

$$
\Lambda_{22}=C\left(s I-A_{0}\right)^{-1} E
$$


with:

$$
A_{0}=\left[\begin{array}{ccc}
a_{11}-\sum_{j=1}^{m} b_{1 j} \frac{k_{j}}{2} c_{j 1} & \ldots & a_{1 n}-\sum_{j=1}^{m} b_{1 j} \frac{k_{j}}{2} c_{j n} \\
\vdots & \ddots & \vdots \\
a_{n 1}-\sum_{j=1}^{m} b_{n j} \frac{k_{j}}{2} c_{j 1} & \ldots & a_{n n}-\sum_{j=1}^{m} b_{n j} \frac{k_{j}}{2} c_{j n}
\end{array}\right]
$$

and:

$$
E=\left[\begin{array}{ccc}
-\frac{k_{1}}{2} b_{11} & \cdots & -\frac{k_{m}}{2} b_{1 m} \\
\vdots & \ddots & \vdots \\
-\frac{k_{1}}{2} b_{n 1} & \cdots & -\frac{k_{m}}{2} b_{n m}
\end{array}\right]
$$

Where $a_{i j}, b_{i j}$ and $c_{i j}$ are coefficients of the matrices $A, B$ and $C$, respectively.

Proof. By Lemma 4, one has the matrix $\Lambda$. Thus, from Figure 15, one has inputs $\left[r_{1} p\right]^{T}$ and outputs $\left[\begin{array}{ll}\sigma & q\end{array}\right]^{T}$, and from (2.23) one obtains the Laplace transform of this system:

$$
\begin{gathered}
x(s)=\left(s I-A_{0}\right)^{-1} B_{0} r_{1}+\left(s I-A_{0}\right)^{-1} E p, \\
\sigma(s)=C_{0}\left(s I-A_{0}\right)^{-1} B_{0} r_{1}+D_{0} r_{1}+F p+C_{0}\left(s I-A_{0}\right)^{-1} E p, \\
q(s)=G\left[\left(s I-A_{0}\right)^{-1} B_{0} r_{1}+G\left(s I-A_{0}\right)^{-1} E p\right]+H r_{1} .
\end{gathered}
$$

With this, one gets:

$$
\left[\begin{array}{l}
\sigma \\
q
\end{array}\right]=\left[\begin{array}{cc}
C_{0}\left(s I-A_{0}\right)^{-1} B_{0}+D_{0} & C_{0}\left(s I-A_{0}\right)^{-1} E+F \\
G\left(s I-A_{0}\right)^{-1} B_{0}+H & G\left(s I-A_{0}\right)^{-1} E
\end{array}\right]\left[\begin{array}{l}
r_{1} \\
p
\end{array}\right] .
$$

From (4.4) one extracts $\Lambda$, like in (2.67):

$$
\Lambda=\left[\begin{array}{cc}
\Lambda_{11}(s) & \Lambda_{12}(s) \\
\Lambda_{21}(s) & \Lambda_{22}(s)
\end{array}\right]=\left[\begin{array}{cc}
C_{0}\left(s I-A_{0}\right)^{-1} B_{0}+D_{0} & C_{0}\left(s I-A_{0}\right)^{-1} E+F \\
G\left(s I-A_{0}\right)^{-1} B_{0}+H & G\left(s I-A_{0}\right)^{-1} E
\end{array}\right]
$$

Finally, the closed-loop system from $r_{1}$ to $\sigma$ is related to the lower linear fractional transformation $\sigma=F_{L}(\Lambda, \Delta) r_{1}$, like in (2.68), where $F_{L}(\Lambda, \Delta)=\Lambda_{11}+\Lambda_{12} \Delta \Lambda_{21}(I-$ $\left.\Lambda_{22} \Delta\right)^{-1}$. Assuming $A_{0}$ Hurwitz, it is guaranteed nominal stability of $\Lambda_{22}$, so by applying Theorem 7 , the system (2.28) is robustly stable if and only if $\mu\left(\Lambda_{22}(j \omega)\right)<1, \quad \forall \omega$. The Lemma 4 provides $G, A_{0}, E$ and ensures that the condition $\bar{\sigma}(\Delta) \leq 1$ is satisfied, and by Lemma 2, for some class of functions in the sector, one has:

Robust Stability of (2.28) $\Rightarrow$ Absolute Stability of (1.2),

which proves the theorem.

Remark 11. Note that $A_{0}$ may not be Hurwitz for a given $k$, but it may be possible to find another $k$ that makes $A_{0}$ Hurwitz. In order to solve this problem, the Routh-Hurwitz 
could be used. Hence, one can proceed with the analysis.

Example 5. Consider the neural network with two neurons (LIAO; WANG; YU, 2007):

$$
\left\{\begin{array}{l}
\dot{x}_{1}=-x_{1}-2 f_{1}\left(x_{1}\right)+1.5 f_{2}\left(x_{2}\right)+I_{1} \\
\dot{x}_{2}=-x_{2}+1.5 f_{1}\left(x_{1}\right)-2 f_{2}\left(x_{2}\right)+I_{2}
\end{array}\right.
$$

where $f_{i}\left(x_{i}\right)=\tanh \left(x_{i}\right), I_{i}=0$ and $i=1,2$. In this example, one has:

$$
A=\left(\begin{array}{cc}
-1 & 0 \\
0 & -1
\end{array}\right), B=\left(\begin{array}{cc}
-2 & 1.5 \\
1.5 & -2
\end{array}\right), C=\left(\begin{array}{ll}
1 & 0 \\
0 & 1
\end{array}\right) .
$$

In order to include tanh $\left(x_{i}\right)$ into a sector, one uses $k_{1}=k_{2}=2$. So, by Theorem 18, this system is absolutely stable according to Figure 4\%. In this figure, one has upper (line) and lower (dash) bounds of $\mu\left(\Lambda_{22}\right)$ and the upper bound is lower than 1 .

Figure 47: $\mu$-analysis for an absolutely stable Lurie type system.

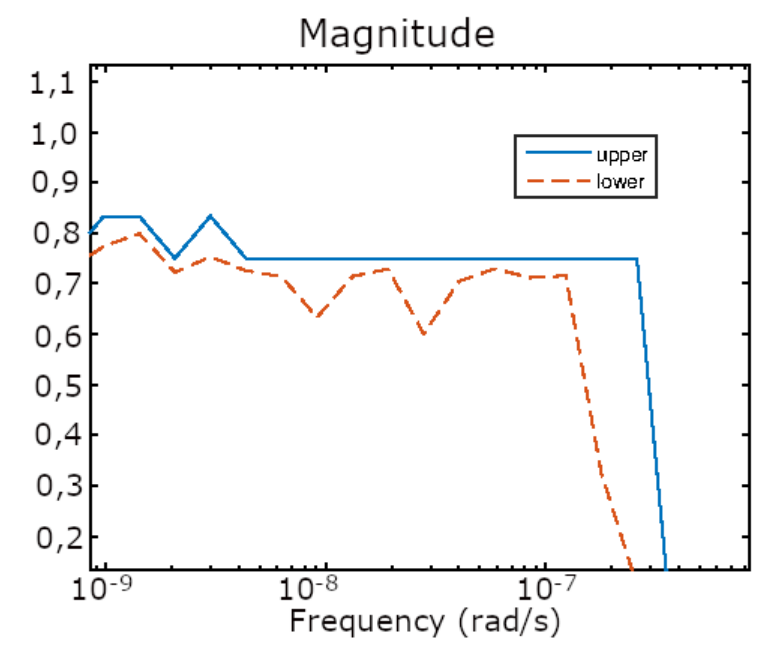

Source: Author.

This conclusion can also be verified by Theorem 2, as presented in the following:

$$
\begin{gathered}
{\left[\begin{array}{cc}
\tilde{a}_{11} & \tilde{a}_{12} \\
\tilde{a}_{21} & \tilde{a}_{22}
\end{array}\right]=\left[\begin{array}{cc}
-1 & 0 \\
0 & -1
\end{array}\right],} \\
\alpha_{i j}=\left[\begin{array}{ll}
\tilde{b}_{11} & \tilde{b}_{12} \\
\tilde{b}_{21} & \tilde{b}_{22}
\end{array}\right]=\left[\begin{array}{ll}
-2 & 1.5 \\
1.5 & -2
\end{array}\right] .
\end{gathered}
$$

Applying the second set of conditions of the Theorem 2, one has that condition 1' of 
the Theorem 2 is satisfied, because $\tilde{a}_{i i}<0$ and the matrix $\left((-1)^{\delta_{i j}}\left|\alpha_{i j}\right|\right)$ has eigenvalues equal to -3.5 and -0.5 so it is Hurwitz.

In relation to condition 2', one has:

$$
\begin{aligned}
& k \tilde{a}_{11} \leq \tilde{b}_{11} \rightarrow k_{1}(-1) \leq(-2), \quad k_{1}\left|\tilde{a}_{21}\right|<\left|\tilde{b}_{21}\right| \rightarrow k_{1}|0|<|1.5|, \\
& \quad \text { for } \quad l=1, \quad i=2 ; \\
& k \tilde{a}_{22} \leq \tilde{b}_{22} \rightarrow k_{2}(-1) \leq(-2), \quad k_{2}\left|\tilde{a}_{12}\right|<\left|\tilde{b}_{12}\right| \rightarrow k_{2}|0|<|1.5|, \\
& \text { for } \quad l=2, \quad i=1 ;
\end{aligned}
$$

taking $k_{1}=k_{2}=2$, the condition 2' is satisfied, ensuring that the equilibrium point is absolutely stable.

Example 6. Consider the neural network of Figure 48. This network was studied by Zak (ZAK, 2003) and Hopfield (HOPFIELD, 1984), being a good example to illustrate the results of this work. The system is represented below:

$$
\left\{\begin{array}{c}
\dot{x}_{1}=-1.4 x_{1}+1.2 f_{2}\left(x_{2}\right)+I_{1}, \\
\dot{x}_{2}=-1.1 x_{2}+f_{1}\left(x_{1}\right)+I_{2},
\end{array}\right.
$$

where $f_{i}\left(x_{i}\right)=\frac{2}{\pi} \arctan \left(1.4 \frac{\pi}{2} x_{i}\right), I_{i}=0$ and $i=1,2$. In this example, one has:

$$
A=\left(\begin{array}{cc}
-1.4 & 0 \\
0 & -1.1
\end{array}\right), B=\left(\begin{array}{cc}
0 & 1.2 \\
1 & 0
\end{array}\right), C=\left(\begin{array}{ll}
0 & 1 \\
1 & 0
\end{array}\right)
$$


Figure 48: Neural network of Example 6.

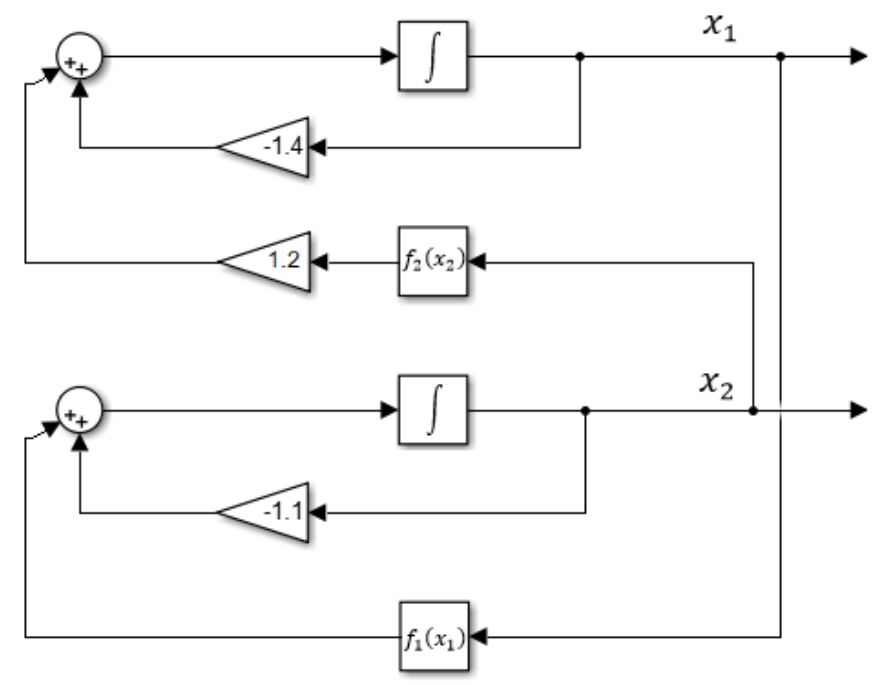

Source: Author.

There is a detailed analysis performed by Zak (ZAK, 2003) about the stability of the neural network in question. He has found three equilibrium points, namely:

$$
x_{1}^{*}=\left(\begin{array}{l}
-0.4098 \\
-0.4245
\end{array}\right), \quad x_{2}^{*}=\left(\begin{array}{l}
0 \\
0
\end{array}\right), \quad \text { e } \quad x_{3}^{*}=\left(\begin{array}{l}
0.4098 \\
0.4245
\end{array}\right) .
$$

Zak concludes in his analysis that the equilibrium points $x_{1}^{*}, x_{3}^{*}$ are asymptotically stable, while the equilibrium point $x_{2}^{*}$ is unstable. There are three equilibrium points and for the system to be absolutely stable, the equilibrium point should be unique. Therefore, the system is not absolutely stable.

As can be seen in Figure 49, in order to include $\frac{2}{\pi} \arctan \left(1.4 \frac{\pi}{2} x_{i}\right)$ into a sector, one uses $k_{1}=k_{2}=2\left(0<f\left(x_{1}\right) \leq k_{1} x_{1}\right.$ and $0<f\left(x_{2}\right) \leq k_{2} x_{2}$ are the sectors $)$. Theorem 18 does not guarantee absolute stability, because $\mu\left(\Lambda_{22}(j w)\right)>1$ according to Figure 50. However, using its criterion, it is possible to find $k_{1}$ and $k_{2}$ that makes the system absolutely stable. So it is found that $k_{\max _{1}}=1.0999, k_{\text {max }_{2}}=1.1666$ and comparing with Theorem 3, one verifies that the developed result has the same degree of conservatism. In verifying Theorem 3 (the generalized Popov criterion), besides the need to find a matrix $N$, and check whether the pair $(\tilde{A}, \tilde{B})$ is controllable and $(\tilde{A}, \tilde{C})$ is observable, as stated by Remark 5, one also needs to do three more checks, that is, one has to check if the matrix $Q(s)$ is asymptotically stable, $H(\infty)>0$, and $H(j \omega)>0$. In this last check, that is shown in Figure 51, it is found that $k_{\max _{1}}=1.0999, k_{\max _{2}}=1.1666$. Therefore, in the developed theorem, only two simple checks are necessary, that is, if $A_{0}$ is Hurwitz and if $\mu<1$. 
Figure 49: Nonlinearity.

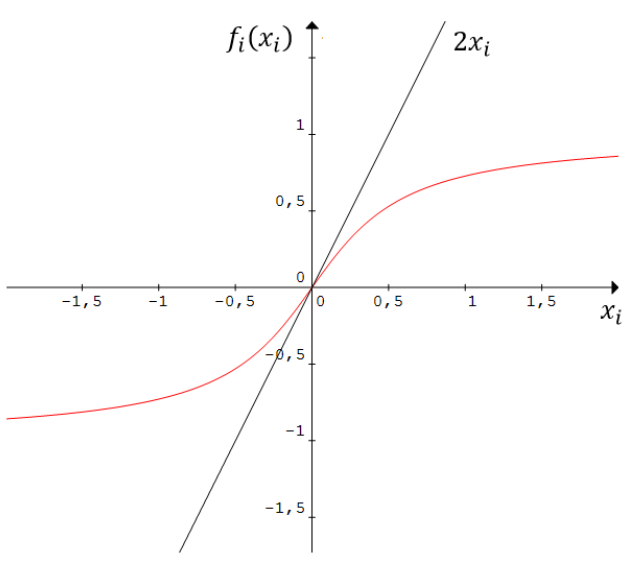

Source: Author.

Figure 50: $\mu$-analysis with sectors $0<f\left(x_{1}\right) \leq k_{1} x_{1}$ and $0<f\left(x_{2}\right) \leq k_{2} x_{2}\left(k_{1}=k_{2}=2\right)$.

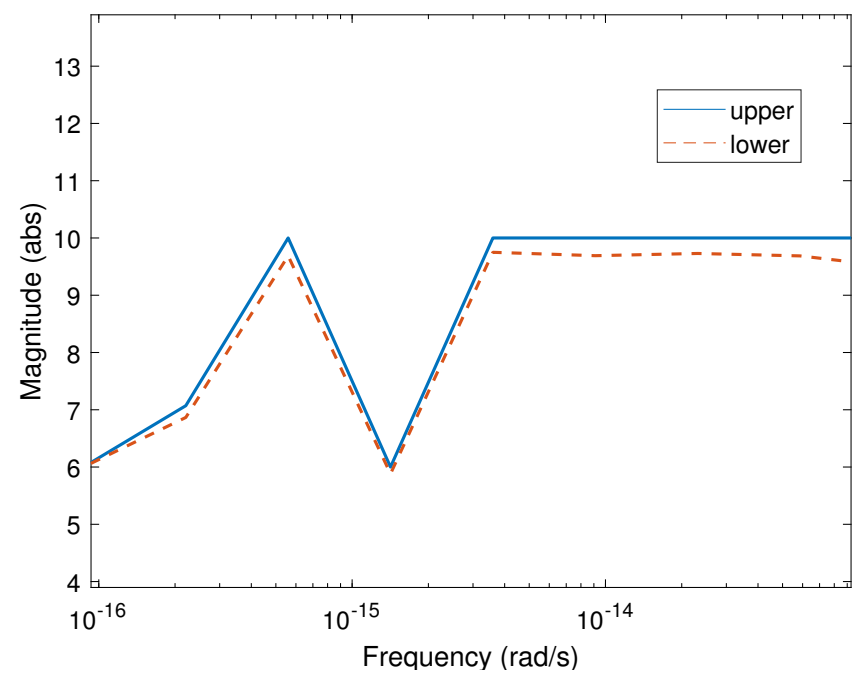

Source: Author. 
Figure 51: Check if $H(j w)>0$ for Theorem 3 .

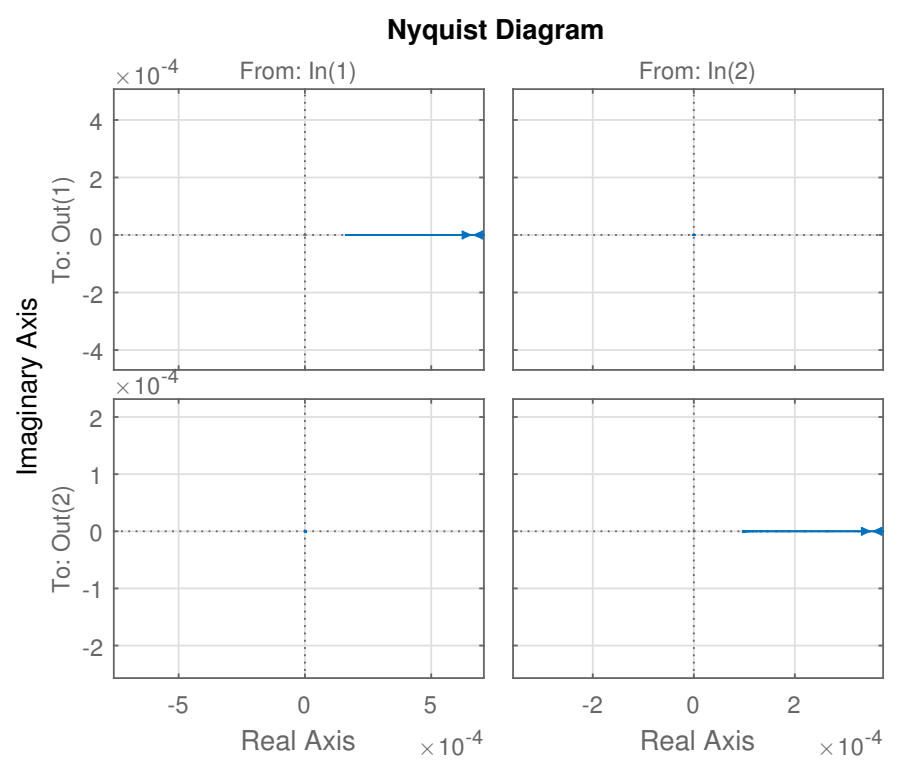

Source: Author.

Figure 52: $\mu$-analysis with $k_{1}=1.0999$ and $k_{2}=1.1666$.

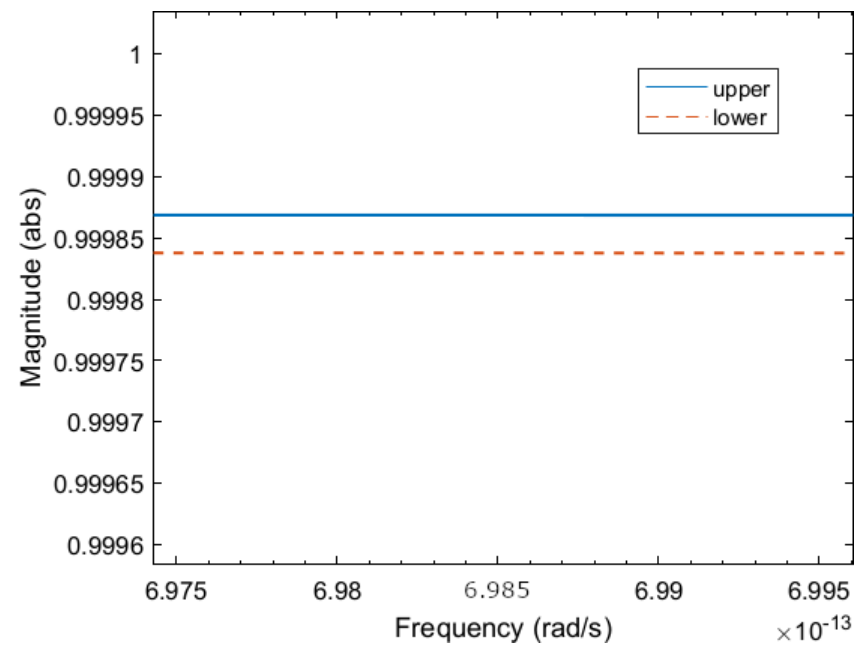

Source: Author.

Remark 12. In Theorems 2 and 3 (that is, Popov criteria), the variables need to be decoupled (matrix $C$ equals identity), and in Theorem 18 this is not necessary. Furthermore, in Theorem 2, it is not possible to determine $k$ (the sector) that stabilizes the system.

Now, Theorem 18 is generalized for the case of sector with two non zero limits.

Theorem 19. Let $\left[\underline{k}_{j}, \bar{k}_{j}\right]$ be the sectors that limit $f_{j}\left(\sigma_{j}\right)$ such that $f_{j} \in F_{\left[k_{j}, \bar{k}_{j}\right]}$ for $j=$ $1, \ldots, m$, and assume that $\bar{A}_{0}$ is Hurwitz. Then, the system (1.2) is absolutely stable for some class of functions in the sector, if: 


$$
\mu\left(\bar{\Lambda}_{22}(j w)\right)<1, \quad \forall w
$$

where:

$$
\bar{\Lambda}_{22}=C\left(s I-\bar{A}_{0}\right)^{-1} \bar{E}
$$

with:

$$
\bar{A}_{0}=\left[\begin{array}{ccc}
a_{11}-\sum_{j=1}^{m} b_{1 j}\left(\frac{\bar{k}_{j}+\underline{k}_{j}}{2}\right) c_{j 1} & \ldots & a_{1 n}-\sum_{j=1}^{m} b_{1 j}\left(\frac{\bar{k}_{j}+\underline{k}_{j}}{2}\right) c_{j n} \\
\vdots & \ddots & \vdots \\
a_{n 1}-\sum_{j=1}^{m} b_{n j}\left(\frac{\bar{k}_{j}+\underline{k}_{j}}{2}\right) c_{j 1} & \ldots & a_{n n}-\sum_{j=1}^{m} b_{n j}\left(\frac{\bar{k}_{j}+\underline{k}_{j}}{2}\right) c_{j n}
\end{array}\right]
$$

and:

$$
\bar{E}=\left[\begin{array}{ccc}
-\left(\frac{\bar{k}_{1}-\underline{k}_{1}}{2}\right) b_{11} & \ldots & -\left(\frac{\bar{k}_{m}-\underline{k}_{m}}{2}\right) b_{1 m} \\
\vdots & \ddots & \vdots \\
-\left(\frac{\bar{k}_{1}-\underline{k}_{1}}{2}\right) b_{n 1} & \ldots & -\left(\frac{\bar{k}_{m}-\underline{k}_{m}}{2}\right) b_{n m}
\end{array}\right]
$$

Where $a_{i j}, b_{i j}$ and $c_{i j}$ are coefficients of the matrices $A, B$ and $C$, respectively.

Proof. The proof of this theorem is analogous to the Theorem 18, with Lemma 4, with a different detail, one must assume that $\underline{k}_{j} \leq \alpha_{j} \leq \bar{k}_{j}$. Thus:

$$
\begin{gathered}
\alpha_{j}=\underline{k}_{j}+\left(\frac{\bar{k}_{j}-\underline{k}_{j}}{2}\right)+\left(\frac{\bar{k}_{j}-\underline{k}_{j}}{2}\right) \delta_{j}=\left(\frac{\bar{k}_{j}+\underline{k}_{j}}{2}\right)+\left(\frac{\bar{k}_{j}-\underline{k}_{j}}{2}\right) \delta_{j}, \quad\left|\delta_{j}\right| \leq 1, \\
\text { for } \quad j=1, \ldots, m .
\end{gathered}
$$

By Lemma 4, one has:

$$
\bar{A}_{0}=\left[\begin{array}{ccc}
a_{11}-\left(b_{11}\left(\frac{\bar{k}_{1}+\underline{k}_{1}}{2}\right) c_{11}\right. & \ldots & a_{1 n}-\left(b_{11}\left(\frac{\bar{k}_{1}+\underline{k}_{1}}{2}\right) c_{1 n}\right. \\
\left.+\ldots+b_{1 m}\left(\frac{\bar{k}_{m}+\underline{k}_{m}}{2}\right) c_{m 1}\right) & & \left.+\ldots+b_{1 m}\left(\frac{\bar{k}_{m}+\underline{k}_{m}}{2}\right) 2 c_{m n}\right) \\
\vdots & \ddots & \vdots \\
a_{n 1}-\left(b_{n 1}\left(\frac{\bar{k}_{1}+\underline{k}_{1}}{2}\right) c_{11}\right. & \ldots & a_{n n}-\left(b_{n 1}\left(\frac{\bar{k}_{1}+\underline{k}_{j}}{2}\right) c_{1 n}\right. \\
\left.+\ldots+b_{n m}\left(\frac{\bar{k}_{m}+\underline{k}_{m}}{2}\right) c_{m 1}\right) & & \left.+\ldots+b_{n m}\left(\frac{\bar{k}_{m}+\underline{k}_{m}}{2}\right) c_{m n}\right)
\end{array}\right]
$$

and:

$$
\bar{A}_{j}=\left[\begin{array}{ccc}
-b_{1 j}\left(\frac{\bar{k}_{j}-\underline{k}_{j}}{2}\right) & \ldots & -b_{1 m}\left(\frac{\bar{k}_{j}-\underline{k}_{j}}{2}\right) c_{m n} \\
\vdots & \ddots & \vdots \\
-b_{n j}\left(\frac{\bar{k}_{j}-\underline{k}_{j}}{2}\right) c_{j 1} & \ldots & -b_{n j}\left(\frac{\bar{k}_{j}-\underline{k}_{j}}{2}\right) c_{j n}
\end{array}\right], \quad j=1, \ldots m
$$

So: 


$$
\bar{E}_{j}=\left[\begin{array}{c}
-\left(\frac{\bar{k}_{j}-\underline{k}_{j}}{2}\right) b_{1 j} \\
\vdots \\
-\left(\frac{\bar{k}_{j}-\underline{k}_{j}}{2}\right) b_{n j}
\end{array}\right] \quad \text { and } \quad \bar{G}_{j}=\left[\begin{array}{lll}
c_{j 1} & \ldots & c_{j n}
\end{array}\right]
$$

Consequently, $\bar{F}_{j}=\bar{H}_{j}=0, \bar{B}_{0}=B, \bar{C}_{0}=C$ and $\bar{D}_{0}=0$. Therefore, one has the $\bar{\Lambda}$-structure based in the Lemma 4 , that is:

$$
\bar{\Lambda}=\left[\begin{array}{ccccc}
\bar{A}_{0} & \bar{B}_{0} & \bar{E}_{1} & \ldots & \bar{E}_{m} \\
\bar{C}_{0} & \bar{D}_{0} & \bar{F}_{1} & \ldots & \bar{F}_{m} \\
\bar{G}_{1} & \bar{H}_{1} & 0 & \ldots & 0 \\
\vdots & \vdots & \vdots & \ddots & \vdots \\
\bar{G}_{m} & \bar{H}_{m} & 0 & \ldots & 0
\end{array}\right]
$$

Henceforward, the proof follows as in Theorem 18, replacing the matrices $A_{0}, B_{0}, C_{0}$, $D_{0}, E_{j}, F_{j}, G_{j}$, and $H_{j}$ with $\bar{A}_{0}, \bar{B}_{0}, \bar{C}_{0}, \bar{D}_{0}, \bar{E}_{j}, \bar{F}_{j}, \bar{G}_{j}$, respectively. This proves the theorem.

\subsection{Time-Delay MIMO Lurie Type Systems}

In this section, one analyzes time-delay Lurie type systems. It is started with Theorem 20 by extending the result of the Theorem 9 to the case of the existence of nonlinearities of type $f_{j} \in F_{\left[\underline{k}_{j}, \bar{k}_{j}\right]}$. Next, the Theorem 21 which in (PINHEIRO; COLÓN, 2021b) was raised as a conjecture is presented. It will be verified that the condition of the Theorem 21, which involves Padé approximations, provides an even less conservative condition compared to Theorem 20. Furthermore, the condition of Theorem 21 is also less conservatives that results of the papers (HE et al., 2005), (LIU et al., 2010), and (GAO; DU; LIU, 2017), which are theorems that bring sufficient conditions to absolute stability and are obtained via LMI, but basically different from the result proposed in the following. The proof of Theorem 21 will be presented in section 4.3.

Lurie type system under consideration is shown in (4.7):

$$
\left\{\begin{array}{l}
\dot{x}=A x-B f(\sigma)+F_{\tau} x(t-\tau)+B r_{1}, \\
\sigma=C x
\end{array}\right.
$$

where $x \in \mathbb{R}^{n}$ is the state vector, $\tau>0$ is a real constant delay, $f(\sigma) \in \mathbb{R}^{m}$ and $\sigma \in \mathbb{R}^{m}$. 
One has the fixed matrices $F_{\tau} \in \mathbb{R}^{n \times n}, B \in \mathbb{R}^{n \times m}, C \in \mathbb{R}^{m \times n}$ and $A \in \mathbb{R}^{n \times n}$ is Hurwitz and fixed.

Theorem 20. Let $\left[\underline{k}_{j}, \bar{k}_{j}\right]$ be the sectors that limit $f_{j}\left(\sigma_{j}\right)$ such that $f_{j} \in F_{\left[\underline{k}_{j}, \bar{k}_{j}\right]}$ for $j=$ $1, \ldots, m$. The system (4.7) is absolutely stable for all delay $\in[0, \tau)$ and for some class of functions in a sector, if $\Lambda_{22}^{\tau}$ is stable and

$$
\mu_{\Delta_{\tau}}\left(W(\omega)\left(\Lambda_{22}^{\tau}(j \omega)\right)<1, \quad \forall \omega\right.
$$

where:

$$
\Lambda_{22}^{\tau}(s)=\left[\begin{array}{cc}
C\left(s I-\bar{A}_{0}^{\tau}\right)^{-1} \bar{E} & C\left(s I-\bar{A}_{0}^{\tau}\right)^{-1} F_{\tau} \\
I\left(s I-\bar{A}_{0}^{\tau}\right)^{-1} \bar{E} & I\left(s I-\bar{A}_{0}^{\tau}\right)^{-1} F_{\tau}
\end{array}\right], \quad W(s)=\left[\begin{array}{cc}
I_{m} & 0 \\
0 & \gamma I_{\rho}
\end{array}\right] .
$$

With: $\rho=\operatorname{rank}(F \tau), \gamma(w)$ a function which bounds the magnitude of $e^{-j w \tau}-1$,

$$
\bar{A}_{0}^{\tau}=\left[\begin{array}{ccc}
a_{11}-\sum_{j=1}^{m} b_{1 j}\left(\frac{\bar{k}_{j}+\underline{k}_{j}}{2}\right) c_{j 1} & \ldots & a_{1 n}-\sum_{j=1}^{m} b_{1 j}\left(\frac{\bar{k}_{j}+\underline{k}_{j}}{2}\right) c_{j n} \\
\vdots & \ddots & \vdots \\
a_{n 1}-\sum_{j=1}^{m} b_{n j}\left(\frac{\bar{k}_{j}+\underline{k}_{j}}{2}\right) c_{j 1} & \ldots & a_{n n}-\sum_{j=1}^{m} b_{n j}\left(\frac{\bar{k}_{j}+\underline{k}_{j}}{2}\right) c_{j n}
\end{array}\right]+F_{\tau},
$$

and:

$$
\bar{E}=\left[\begin{array}{ccc}
-\left(\frac{\bar{k}_{1}-\underline{k}_{1}}{2}\right) b_{11} & \ldots & -\left(\frac{\bar{k}_{m}-\underline{k}_{m}}{2}\right) b_{1 m} \\
\vdots & \ddots & \vdots \\
-\left(\frac{\bar{k}_{1}-\underline{k}_{1}}{2}\right) b_{n 1} & \ldots & -\left(\frac{\bar{k}_{m}-\underline{k}_{m}}{2}\right) b_{n m}
\end{array}\right]
$$

Where $a_{i j}, b_{i j}$ and $c_{i j}$ are coefficients of the matrices $A, B$ and $C$, respectively.

Proof. By Equation (4.2) of the Lemma 4 (with $\bar{A}_{0}$ equal to that given by the Theorem 19 , because here $\left.f_{j} \in F_{\left[\underline{k}_{j}, \bar{k}_{j}\right]}\right)$ and adding the term $F_{\tau} x-F_{\tau} x$, the Equation (4.7) can be rewritten as follows:

$$
\dot{x}=\left(\bar{A}_{0}+\sum_{j=1}^{m} \delta_{j} A_{j}\right) x+B r_{1}+F_{\tau} x(t-\tau)+F_{\tau} x-f_{\tau} x,
$$

and one has:

$$
\dot{x}=\left(\bar{A}_{0}+F_{\tau}+\sum_{j=1}^{m} \delta_{j} A_{j}\right) x+B r_{1}+F_{\tau}(x(t-\tau)-x) .
$$

Doing $\bar{A}_{0}^{\tau}=\bar{A}_{0}+F_{\tau}$ and $\mathcal{D}_{\tau} x=x(t-\tau)$, one has:

$$
\dot{x}=\left(\bar{A}_{0}^{\tau}+\sum_{j=1}^{m} \delta_{j} A_{j}\right) x+B r_{1}+F_{\tau}\left(\mathcal{D}_{\tau}-1\right) I x .
$$

Now, gathering the $\Lambda$ - $\Delta$-Structure from the Lemma 4 (see Figure 15) with the structure of 
the Equation 2.79 (see Figure 26), it is obtained a new structure in according to Equation (4.9) and Figure 53.

$$
\left[\begin{array}{l}
\dot{x} \\
\sigma \\
q_{1} \\
\vdots \\
q_{m} \\
y
\end{array}\right]=\underbrace{\left[\begin{array}{cccccc}
\bar{A}_{0}^{\tau} & B & E_{1} & \ldots & E_{m} & F_{\tau} \\
C_{0} & D_{0} & F_{1} & \ldots & F_{m} & 0 \\
G_{1} & H_{1} & 0 & \ldots & 0 & 0 \\
\vdots & \vdots & \vdots & \ddots & \vdots & \vdots \\
G_{m} & H_{m} & 0 & \ldots & 0 & 0 \\
I & 0 & 0 & \ldots & 0 & 0
\end{array}\right]}_{\Lambda^{\tau}}\left[\begin{array}{l}
x \\
r_{1} \\
p_{1} \\
\vdots \\
p_{m} \\
u
\end{array}\right]
$$

where by Lemma 4: $C_{0}=G=C, D_{0}=F=H=0$; and by the Theorem 19: $E=\bar{E}$.

Figure 53: $\Lambda-\Delta-\mathcal{D}$-Structure

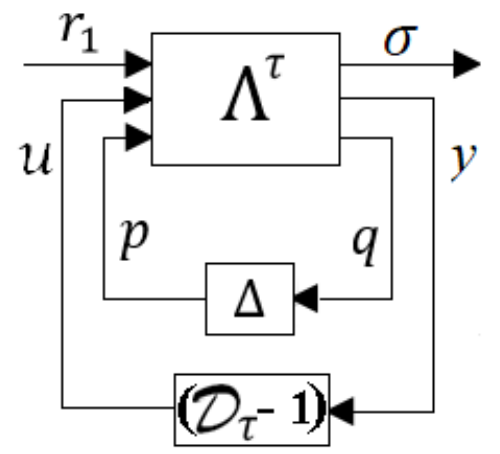

Source: Author.

Proceeding with the development of Equation 4.9, one obtains:

$$
\left\{\begin{aligned}
\dot{x} & =\bar{A}_{0}^{\tau} x+B r_{1}+\bar{E} p+F_{\tau} u \\
\sigma & =C x \\
q & =C x \\
z & =I x
\end{aligned}\right.
$$

Obtaining the Laplace transform:

$$
\left\{\begin{aligned}
s X(s) & =\bar{A}_{0}^{\tau} X(s)+B r_{1}(s)+\bar{E} p(s)+F_{\tau} u(s), \\
\sigma(s) & =C X(s), \\
q(s)= & C X(s), \\
y(s)= & I X(s) .
\end{aligned}\right.
$$

From the first equation of (4.11), one has: 


$$
X(s)=\left(s I-\bar{A}_{0}^{\tau}\right)^{-1} B r_{1}(s)+\left(s I-\bar{A}_{0}^{\tau}\right)^{-1} E p(s)+\left(s I-\bar{A}_{0}^{\tau}\right) F_{\tau} u(s) .
$$

Substituting $X(s)$ into the second, third and fourth equations of (4.11), one has:

$$
\begin{aligned}
& \sigma(s)=\left[C\left(s I-\bar{A}_{0}^{\tau}\right)^{-1} B\right] r_{1}(s)+\left[C\left(s I-\bar{A}_{0}^{\tau}\right)^{-1} E\right] p(s)+\left[C\left(s I-\bar{A}_{0}^{\tau}\right)^{-1} F_{\tau}\right] u(s), \\
& q(s)=\left[C\left(s I-\bar{A}_{0}^{\tau}\right)^{-1} B\right] r_{1}(s)+\left[C\left(s I-\bar{A}_{0}^{\tau}\right)^{-1} E\right] p(s)+\left[C\left(s I-\bar{A}_{0}^{\tau}\right)^{-1} F_{\tau}\right] u(s),
\end{aligned}
$$

and

$$
y(s)=\left[I\left(s I-\bar{A}_{0}^{\tau}\right)^{-1} B\right] r_{1}(s)+\left[I\left(s I-\bar{A}_{0}^{\tau}\right)^{-1} E\right] p(s)+\left[I\left(s I-\bar{A}_{0}^{\tau}\right)^{-1} F_{\tau}\right] u(s) .
$$

Thus, it is obtained the LFT with inputs $\left[\begin{array}{lll}r_{1} & p & u\end{array}\right]^{T}$ and outputs $\left[\begin{array}{lll}\sigma & q & y\end{array}\right]^{T}$ :

$$
\left[\begin{array}{c}
\sigma \\
q \\
y
\end{array}\right]=\left[\begin{array}{c|cc}
C\left(s I-\bar{A}_{0}^{\tau}\right)^{-1} B & C\left(s I-\bar{A}_{0}^{\tau}\right)^{-1} E & C\left(s I-\bar{A}_{0}^{\tau}\right)^{-1} F_{\tau} \\
\hline C\left(s I-\bar{A}_{0}^{\tau}\right)^{-1} B & C\left(s I-\bar{A}_{0}^{\tau}\right)^{-1} E & C\left(s I-\bar{A}_{0}^{\tau}\right)^{-1} F_{\tau} \\
I\left(s I-\bar{A}_{0}^{\tau}\right)^{-1} B & I\left(s I-\bar{A}_{0}^{\tau}\right)^{-1} E & I\left(s I-\bar{A}_{0}^{\tau}\right)^{-1} F_{\tau}
\end{array}\right]\left[\begin{array}{l}
r_{1} \\
p \\
u
\end{array}\right]
$$

From (4.12) one has $\Lambda^{\tau}$, like in (2.67):

$$
\Lambda^{\tau}=\left[\begin{array}{cc}
\Lambda_{11}^{\tau}(s) & \Lambda_{12}^{\tau}(s) \\
\Lambda_{21}^{\tau}(s) & \Lambda_{22}^{\tau}(s)
\end{array}\right]
$$

then

$$
\Lambda_{22}^{\tau}(s)=\left[\begin{array}{cc}
C\left(s I-\bar{A}_{0}^{\tau}\right)^{-1} E & C\left(s I-\bar{A}_{0}^{\tau}\right)^{-1} F_{\tau} \\
I\left(s I-\bar{A}_{0}^{\tau}\right)^{-1} E & I\left(s I-\bar{A}_{0}^{\tau}\right)^{-1} F_{\tau}
\end{array}\right] .
$$

In relation to uncertainty of the delay, in Laplace one has $\left(\mathcal{D}_{\tau}-1\right)=\left(e^{-\tau s}-1\right)$. The magnitude of this uncertainty can be bounded by a function $\gamma(w)$. In this case, one can choose one of the equation (2.80), (2.81), (2.82), (2.83) or (2.84). Thus, it is guaranteed that $\gamma(\omega) \leq\left|\gamma^{*}(s)\right|$, furthermore, in according to Lemma 4 the normalized uncertainties for the nonlinearities are $\delta_{i}$ for $i=1, \ldots m$. Therefore,

$$
\Delta_{\tau}=\operatorname{diag}\{\underbrace{\delta_{1}, \ldots, \delta_{m}}_{\Delta},\left(\mathcal{D}_{\tau}-1\right) I_{\rho}\}
$$

and consequently

$$
W=\operatorname{diag}\left\{I_{m}, \gamma^{*} I_{\rho}\right\}
$$

where $\rho=\operatorname{rank}\left(F_{\tau}\right)$ (see Equation (2.79), where here was chosen $C_{i}=I$ and $B_{i}=F_{\tau}$ ).

The closed-loop system from $r_{1}$ to $\sigma$ is related to the lower linear fractional transforma- 
tion $\sigma=F_{L}\left(\Lambda^{\tau}, \Delta_{\tau}\right) r_{1}$, like in (2.68), where $F_{L}\left(\Lambda^{\tau}, \Delta_{\tau}\right)=\Lambda_{11}^{\tau}+\Lambda_{12}^{\tau} \Delta_{\tau} \Lambda_{21}^{\tau}\left(I-\Lambda_{22}^{\tau} \Delta_{\tau}\right)^{-1}$. By Theorem 7 , for the stability of $F_{L}\left(\Lambda^{\tau}, \Delta_{\tau}\right)$, the $\mu$-analysis of $\Lambda_{22}^{\tau}$ is sufficient, as well as, must be guaranteed the nominal stability of $\Lambda_{22}$, and finally, one applies the Theorem 9. By the Equation (4.9) and Figure 53 the system (4.7) is putted as a robust control problem with the nonlinearities and delay modeled through uncertainties. Therefore, the condition 4.8, if satisfied, guarantees absolute stability for the system (4.7), which proves the theorem.

The following theorem (presented as conjecture in (PINHEIRO; COLÓN, 2021b)), provides a sufficient condition for absolute stability of the system (4.7), that is less conservative than the other theorems for delay developed in this thesis and the presented in others works. It is an extension of the result of Theorem 19, where delay is now inserted.

Theorem 21. Suppose that $f_{j} \in F_{\left[\underline{k}_{j}, \bar{k}_{j}\right]}$ for $j=1, \ldots, m$, and assume that $\bar{\Lambda}_{22, \text { delay }}$ with the delay term replaced by the Padé approximations of all order are stable. Then the system (4.7) is absolutely stable for some class of functions in the sector, if:

$$
\mu\left(\bar{\Lambda}_{22, k}(j \omega)\right)<1, \quad \forall \omega, \forall k \in \mathbb{Z}^{+},
$$

where:

$$
\begin{aligned}
\bar{\Lambda}_{22, \text { delay }} & =C\left(s I-\bar{A}_{0}-F_{\tau} e^{-\tau s}\right)^{-1} \bar{E}, \\
\bar{\Lambda}_{22, k}(j \omega) & =C\left(j \omega I-\bar{A}_{0}-F_{\tau}[k / k]\right)^{-1} \bar{E},
\end{aligned}
$$

with:

$$
\bar{A}_{0}=\left[\begin{array}{ccc}
a_{11}-\sum_{j=1}^{m} b_{1 j}\left(\frac{\bar{k}_{j}+\underline{k}_{j}}{2}\right) c_{j 1} & \ldots & a_{1 n}-\sum_{j=1}^{m} b_{1 j}\left(\frac{\bar{k}_{j}+\underline{k}_{j}}{2}\right) c_{j n} \\
\vdots & \ddots & \vdots \\
a_{n 1}-\sum_{j=1}^{m} b_{n j}\left(\frac{\bar{k}_{j}+\underline{k}_{j}}{2}\right) c_{j 1} & \ldots & a_{n n}-\sum_{j=1}^{m} b_{n j}\left(\frac{\bar{k}_{j}+\underline{k}_{j}}{2}\right) c_{j n}
\end{array}\right]
$$

and:

$$
\bar{E}=\left[\begin{array}{ccc}
-\left(\frac{\bar{k}_{1}-\underline{k}_{1}}{2}\right) b_{11} & \ldots & -\left(\frac{\bar{k}_{m}-\underline{k}_{m}}{2}\right) b_{1 m} \\
\vdots & \ddots & \vdots \\
-\left(\frac{\bar{k}_{1}-\underline{k}_{1}}{2}\right) b_{n 1} & \ldots & -\left(\frac{\bar{k}_{m}-\underline{k}_{m}}{2}\right) b_{n m}
\end{array}\right]
$$

Where $a_{i j}, b_{i j}$ and $c_{i j}$ are coefficients of the matrices $A, B$ and $C$, respectively.

Proof. As the proof is large, section 4.3 is dedicated to it. 
Remark 13. In principle, it would be necessary to check for all Padé approximants. On the other hand, if some successive terms of the series indexed by $k$ satisfy the condition, the prediction of absolute stability can be accepted.

Remark 14. According to Skogestad (SKOGESTAD; POSTLETHWAITE, 2007) the weights in the generalized plant $P$ need not even be rational transfer functions, thus, in the same way for the case presented here, the matrix $\bar{\Lambda}$ can be irrational, and its use in software should be by means of approximations. Here, it is used Padé approximations.

In the following, the theorem is applied in an example:

Example 7. Consider the same Chua's circuit (4.16) of the example 2. This circuit was also studied by Gao (GAO; DU; LIU, 2017):

$$
\left\{\begin{array}{l}
\dot{x_{1}}(t)=\alpha\left[-x_{1}(t)+x_{2}-f\left(x_{1}(t)\right)\right]+F_{11} x(t-\tau)+F_{12} x(t-\tau)+F_{13} x(t-\tau), \\
\dot{x_{2}}(t)=x_{1}(t)-x_{2}(t)+x_{3}(t)+F_{21} x(t-\tau)+F_{22} x(t-\tau)+F_{23} x(t-\tau), \\
\dot{x_{3}}(t)=-\beta x_{2}(t)-\vartheta x_{3}(t)+F_{31} x(t-\tau)+F_{32} x(t-\tau)+F_{33} x(t-\tau),
\end{array}\right.
$$

where $x_{1}(t), x_{2}(t)$ and $x_{3}(t)$ are state variables, $f\left(x_{1}(t)\right)=m_{1} x_{1}(t)+\frac{1}{2}\left(m_{0}-m_{1}\right)\left(\mid x_{1}(t)+\right.$ $\left.1|-| x_{1}(t)-1 \mid\right)$, and $\alpha, \beta, \vartheta, m_{0}$ and $m_{1}$ are real numbers. Furthermore, $m_{0}$ and $m_{1}$ are bounds of the sector. In this example, inserting the delay $x(t-\tau)$ and putting Chua's system in the form of (4.7), one has:

$$
\begin{gathered}
A=\left[\begin{array}{ccc}
-\alpha & \alpha & 0 \\
1 & -1 & 1 \\
0 & -\beta & \vartheta
\end{array}\right], \quad B=\left[\begin{array}{c}
-\alpha \\
0 \\
0
\end{array}\right], \quad F_{\tau}=\left[\begin{array}{ccc}
-0.8 & 0.5 & 3.6 \\
0.4 & 0.1 & -0.1 \\
0.4 & 0.01 & -0.8
\end{array}\right] \\
C=\left[\begin{array}{l}
1 \\
0 \\
0
\end{array}\right], \quad \sigma(t)=x_{1}(t) .
\end{gathered}
$$

The example analyzes the maximum value of the delay ( $\left.\tau_{\max }\right)$, that is, the maximum delay in $x(t-\tau)$ so that the system remains absolutely stable. In simulations, the parameters of the time-delay Chua's circuit are set as $\alpha=-1.3018, \beta=-0.0136, \vartheta=-0.0297$, $m_{0}=0.1691, m_{1}=-0.4768$. As the Padé approximation is used to approximate the delay in the condition (4.15), the Table 6 relates the $\tau_{\max }$ to the Padé approximation order.

Remark 15. For the Padé approximation of first order, $\tau_{\max }$ obtained is $\tau_{\max }=0.7476$. With higher values of $\tau$, the system becomes unstable (Figure 54). However, using the second order or higher approximation, $\tau_{\max }$ approaches 0.683875 , the system remains stable, as can be seen in Figure 56. 
Table 6: $\tau_{\max }$ and Padé approximation order.

\begin{tabular}{lllllllll}
\hline Order & 1 & 2 & 3 & 4 & $\ldots$ & 10 & $\ldots$ & 20 \\
\hline$\tau_{\max }$ & 0.7476 & 0.6847 & 0.68388 & 0.683875 & $\ldots$ & $0.683875^{*}$ & $\ldots$ & $0.683875^{*}$ \\
\hline \multicolumn{6}{c}{ *Truncated in this decimal place. } \\
Source: Author.
\end{tabular}

Table 7: $\tau_{\max }$ in different theorems.

\begin{tabular}{lccllc}
\hline $\begin{array}{l}\text { Theo. } 7 \text { in } \\
(\text { HE et al., 2005) }\end{array}$ & Theo. 20 & Theo. 15 & $\begin{array}{l}\text { Theo. } 1 \text { in } \\
\text { (LIU et al., 2010) })\end{array}$ & $\begin{array}{l}\text { Theo. } 1 \text { in } \\
\text { (GAO; DU; LIU, 2017) })\end{array}$ & Theo. 21 \\
\hline 0.0284 & 0.29 & 0.4206 & 0.4517 & 0.6662 & 0.6838 \\
\hline & \multicolumn{5}{c}{ Source: Author. }
\end{tabular}

The Table 7 shows comparisons with other theorems developed in this thesis and results of other papers, showing that the condition (4.15) is less conservative. The analysis of the circuit via condition (4.15) provides $\tau_{\max }=0.6838$ and a $\mu$ value equal to 0.4159 (Figure 55), ensuring that the systems is absolutely stable (Figure 56).

Figure 54: Chua's circuit oscillations.

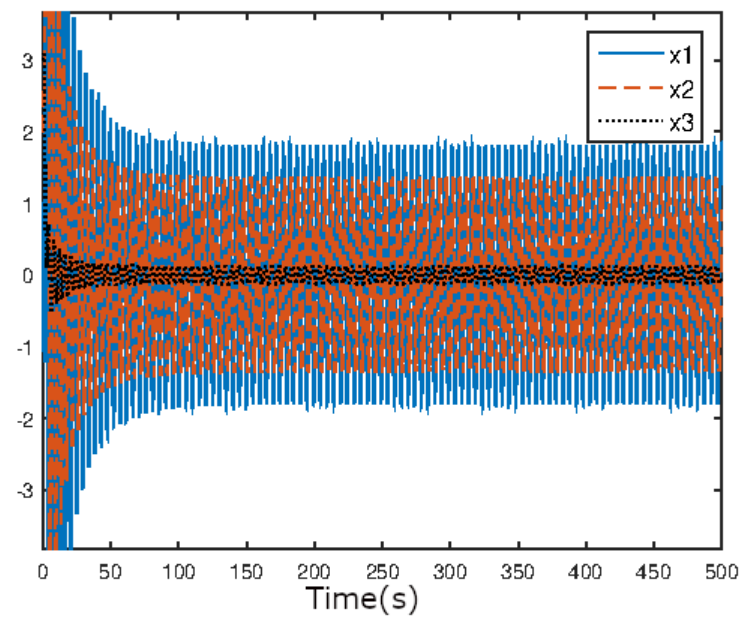

Source: Author. 
Figure 55: $\mu$-analisys.

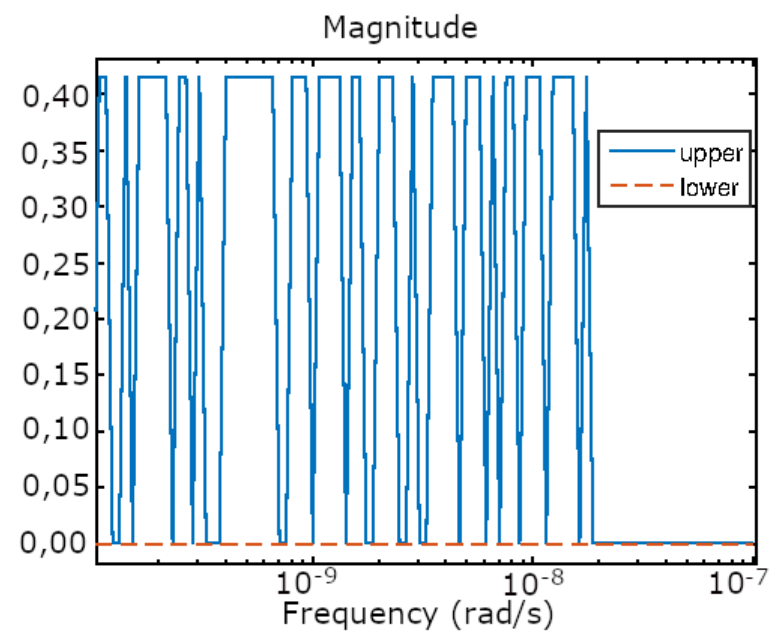

Source: Author.

Figure 56: Absolute stability to Chua's circuit.

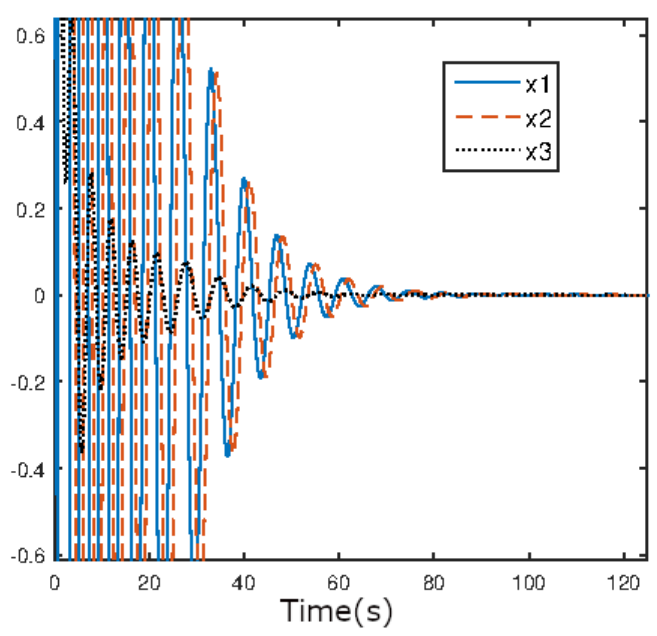

Source: Author.

Remark 16. When the system has time-delay, Padé approximation is used. If the Padé approximation is of order $n$, the system order become $n$, that is, the number of lines and columns of matrix A become $n$ (also others matrices increase). In the limit, that is, when $n \rightarrow \infty$ (that is, when no Padé approximation is used), theoretically the system would be infinite dimensional, which requires the theory of functional differential equations.

Remark 17. Complex networks can be modeled by using graph theory (BOCCALETTI et al., 2006; DING; WANG; HAN, 2020). This work's results can be used to analyse any type of complex network in which there are Lurie type systems in the nodes and which exchange signals by the edges (with or without time-delay). The complete system of 
ordinary differential equations that model the dynamic of the network is a MIMO Lurie type system.

\subsection{Proof of the Theorem 21}

Theorem 21 was presented in the previous section, and it was also published, as conjecture, in (PINHEIRO; COLÓN, 2021b). The theorem is valid for a general linear system that is robustly stable. In this section, the proof will be made. In order to obtain the proof, some theorems about the Padé approximation and its convergence, and LFT theories are needed.

The Theorem 21 is an extension of the Theorem 19, where here the delay is inserted. Thus, by Lemma 4, for the system (4.7) without delay is:

$$
\left\{\begin{aligned}
\dot{x} & =\bar{A}_{0} x+\bar{B}_{0} r_{1}+\bar{E} p \\
\sigma & =\bar{C}_{0} x+\bar{D}_{0} r_{1}+\bar{F} p \\
q & =\bar{G} x+\quad \bar{H} r_{1}
\end{aligned}\right.
$$

Now, inserting the delay, one has:

$$
\left\{\begin{array}{l}
\dot{x}=\bar{A}_{0} x+\bar{B}_{0} r_{1}+\bar{E} p+F_{\tau} x(t-\tau) \\
\sigma=\bar{C}_{0} x+\bar{D}_{0} r_{1}+\bar{F} p \\
q=\bar{G} x+\bar{H} r_{1}
\end{array}\right.
$$

Obtaining the Laplace transform:

$$
\left\{\begin{array}{lll}
s X(s) & =\bar{A}_{0} X(s)+\bar{B}_{0} r_{1}(s)+\bar{E} p(s)+F_{\tau} e^{-\tau s} X(s) \\
\sigma(s) & =\bar{C}_{0} X(s)+\bar{D}_{0} r_{1}(s)+\bar{F} p(s) \\
q(s) & =\bar{G} X(s)+ & \bar{H} r_{1}(s)
\end{array}\right.
$$

From the first equation of (4.19), one has:

$$
X(s)=\left(s I-\bar{A}_{0}-F_{\tau} e^{-\tau s}\right)^{-1} \bar{B}_{0} r_{1}(s)+\left(s I-\bar{A}_{0}-F_{\tau} e^{-\tau s}\right)^{-1} E p(s) .
$$

Substituting $X(s)$ into the second and third equation of (4.19), one gets:

$$
\sigma(s)=\left[\bar{C}_{0}\left(s I-\bar{A}_{0}-F_{\tau} e^{-\tau s}\right)^{-1} \bar{B}_{0}+\bar{D}_{0}\right] r_{1}(s)+\left[\bar{C}_{0}\left(s I-\bar{A}_{0}-F_{\tau} e^{-\tau s}\right)^{-1} \bar{E}+\bar{F}\right] p(s)
$$


and

$$
q(s)=\left[\bar{G}\left(s I-\bar{A}_{0}-F_{\tau} e^{-\tau s}\right)^{-1} \bar{B}_{0}+\bar{H}\right] r_{1}(s)+\bar{G}\left(s I-\bar{A}_{0}-F_{\tau} e^{-\tau s}\right)^{-1} \bar{E} p(s) .
$$

Thus, the LFT is obtained with inputs $\left[r_{1} p\right]^{T}$ and outputs $\left[\begin{array}{ll}\sigma & q\end{array}\right]^{T}$ :

$$
\left[\begin{array}{l}
\sigma \\
q
\end{array}\right]=\left[\begin{array}{cc}
\bar{C}_{0}\left(s I-\bar{A}_{0}-F_{\tau} e^{-\tau s}\right)^{-1} \bar{B}_{0}+\bar{D}_{0} & \bar{C}_{0}\left(s I-\bar{A}_{0}-F_{\tau} e^{-\tau s}\right)^{-1} \bar{E}+\bar{F} \\
\bar{G}\left(s I-\bar{A}_{0}-F_{\tau} e^{-\tau s}\right)^{-1} \bar{B}_{0}+\bar{H} & \bar{G}\left(s I-\bar{A}_{0}-F_{\tau} e^{-\tau s}\right)^{-1} \bar{E}
\end{array}\right]\left[\begin{array}{l}
r_{1} \\
p
\end{array}\right] .
$$

From (4.20) and $\bar{G}=C$ (Lemma 4), one has $\bar{\Lambda}_{\text {delay }}$, like in (2.67):

$$
\begin{gathered}
\bar{\Lambda}_{\text {delay }}=\left[\begin{array}{cc}
\bar{\Lambda}_{11, \text { delay }}(s) & \bar{\Lambda}_{12, \text { delay }}(s) \\
\bar{\Lambda}_{21, \text { delay }}(s) & \bar{\Lambda}_{22, \text { delay }}(s)
\end{array}\right]= \\
{\left[\begin{array}{cc}
\bar{C}_{0}\left(s I-\bar{A}_{0}-F_{\tau} e^{-\tau s}\right)^{-1} \bar{B}_{0}+\bar{D}_{0} & \bar{C}_{0}\left(s I-\bar{A}_{0}-F_{\tau} e^{-\tau s}\right)^{-1} \bar{E}+\bar{F} \\
C\left(s I-\bar{A}_{0}-F_{\tau} e^{-\tau s}\right)^{-1} \bar{B}_{0}+\bar{H} & C\left(s I-\bar{A}_{0}-F_{\tau} e^{-\tau s}\right)^{-1} \bar{E}
\end{array}\right] .}
\end{gathered}
$$

From now on, it will be worked with Padé approximations, where $M=L$, i.e., the degree of the numerator is equal to that of the denominator. When the exponential is replaced by a Padé approximation, a sequence of rational matrices will be obtained, whose degree is increasing, both of the numerators and the denominators, which are represented by:

$$
\bar{\Lambda}_{22, k}(j \omega)=C\left(j \omega I-\bar{A}_{0}-F_{\tau}[k / k]\right)^{-1} \bar{E} .
$$

In the limit, one has a matrix with an infinite number of poles and zeros. For each $k$ of this sequence of matrices, one has a sequence of functions that are the structured singular values:

$$
\mu_{k}(j \omega)=\mu_{\Delta}\left(\bar{\Lambda}_{22, k}(j \omega)\right)
$$

where $\Delta$ is a diagonal matrix, which represents the uncertainty structure of Lurie's problem.

In principle, the goal would be to verify:

- If the sequence of function (4.23) converges.

Thus, it is necessary to analyze the convergence of the sequence of complex functions $\mu_{k}(s)$. In particular, one wants to know if this sequence of functions converges. According to (DOYLE, 1982) the $\mu$ function is continuous. So, it is necessary to know if the limit of this sequence is a continuous function.

Let us define the function $\Phi(s)=s I-\bar{A}_{0}-F_{\tau} e^{-s t}$ and the sequence of functions $\Phi_{k}(s)=s I-\bar{A}_{0}-F_{\tau}[k / k]$. 
Lemma 5. Let be the sequence of maps: $\Phi_{k}(s)=s I-\bar{A}_{0}-F_{\tau}[k / k]$ which also forms a sequence of rational matrices. This sequence of matrices of rational functions is point-topoint convergent.

Proof. Each entry of $F_{\tau}[k / k]$ is a rational functions. By the Theorem 11, this sequences is uniformly convergent, so the matrix sequence is convergent $F_{\tau} e^{-s \tau}$. Since $s I-\bar{A}_{0}$ can be thought as a constant sequence (which always converges), and the sum of two convergent sequences of complex matrices always converges, then $\left\{\Phi_{k}(s)\right\}$ converges to $\Phi(s)$ then the result follows.

Lemma 6. The sequence of matrices of rational functions $\left\{\Phi_{k}(s)\right\}$ converges uniformly to the matrix of holomorphic functions $s I-\bar{A}_{0}-F_{\tau} e^{-\tau s}$.

Proof. We know from the Theorem 11 that the sequence of Padé approximations $[k / k]$ converges uniformly to $e^{-\tau s}$. The matrix sequence $\left\{F_{\tau}[k / k]\right\}$ is then formed by entries that are uniformly convergent. Using the $\infty$-norm (Equation (2.7)), which is not the $H_{\infty}$ norm used in $H_{\infty}$ control, one has that:

$$
\begin{aligned}
& \left\|\Phi_{k}(s)-\Phi(s)\right\|_{\infty}=\left\|s I-\bar{A}_{0}-F_{\tau}[k / k]-s I+\bar{A}_{0}+F_{\tau} e^{-\tau s}\right\|_{\infty}= \\
& =\left\|F_{\tau}\left(e^{-\tau s}-[k / k]\right)\right\|_{\infty}=\max _{i}\left(\sum_{j}\left|\left[F_{\tau}\right]_{i j}\right|\left|[k / k]-e^{-\tau s}\right|\right)=\left\|F_{\tau}\right\|_{\infty}\left|e^{-\tau s}-[k / k]\right| .
\end{aligned}
$$

Since for every $\epsilon>0$, there exists $K>0$ such that for all $k>K,\left|[k / k]-e^{-\tau s}\right|<\epsilon$, one can always choose a $\epsilon_{2}>0$ such that there is a $\epsilon=\epsilon_{2} /\left\|F_{\tau}\right\|_{\infty}$ such that there is a $K>0$ such that all $k>K$. Then, one has:

$$
\left\|\Phi_{k}(s)-\Phi(s)\right\|_{\infty}=\left\|F_{\tau}\right\|_{\infty} \epsilon<\epsilon_{2}
$$

then $\left\{\Phi_{k}(s)\right\}$ converges uniformly for the norm in question. Since the space of matrices is of finite dimension, then if a sequence converges in one norm, it converges in all other norms of the same space, which proves the theorem.

One needs now to define the sequence $\left\{\Phi_{k}^{-1}(s)\right\}$ and to know if it is uniformly convergent.

Lemma 7. The sequence of rational matrices $\left\{\Phi_{k}^{-1}(s)\right\}$ is point-to-point convergent, except in zero measured set of points.

Proof. The sequence $\Phi_{k}(s)$ is convergent. Also, $f(A)=A^{-1}$ is continuous if and only if $A \in G L(\mathbb{C}, n)$ which is the group of $n \times n$ complex matrices with determinant not zero. 
That is, in an open set in which $f$ is continuous. Let us exclude the finite set of poles where $\Phi_{k}(s)$ is not defined. Due to the fact that the entries of the matrices $\Phi_{k}(s)$ are meromorphic, this set of points has also zero measure in $\mathbb{C}$, so every $\Phi_{k}(s)$ is defined almost everywhere in $\mathbb{C}$ (that is, in a open set). The function $f$ obviously is defined in such open set and is continuous, then $\left\{\Phi_{k}^{-1}(s)\right\}$ converges in this set, and the result follows.

Lemma 8. The sequence of rational matrices $\left\{\Phi_{k}^{-1}(j \omega)\right\}$ is uniformly convergent almost everywhere.

Proof. One must prove that for each $\epsilon_{2}>0$, there exists $K>0$ such that for $k>K$, then $\left\|\Phi_{k}^{-1}(s)-\Phi^{-1}(s)\right\|_{\infty}<\epsilon_{2}$. By using the identity $S^{-1}-T^{-1}=-S^{-1}(S-T) T^{-1}$, one has (in the open set described above, that is, almost everywhere):

$$
\begin{aligned}
& \left\|\Phi_{k}^{-1}(s)-\Phi^{-1}(s)\right\|_{\infty} \leq \\
& \left\|\left(s I-\overline{A_{0}}-F_{\tau}[k / k]\right)^{-1}\right\|_{\infty}\left\|F_{\tau}\left([k / k]-e^{-s \tau}\right)\right\|_{\infty}\left\|s I-\overline{A_{0}}-F_{\tau} e^{-s \tau}\right\|_{\infty}= \\
& =\left|[k / k]-e^{-s \tau}\right| \underbrace{\left\|\left(s I-\overline{A_{0}}-F_{\tau}[k / k]\right)^{-1}\right\|_{\infty}\left\|F_{\tau}\right\|_{\infty}\left\|\left(s I-\overline{A_{0}}-F_{\tau} e^{-s \tau}\right)^{-1}\right\|_{\infty}}_{=\Gamma(s)} .
\end{aligned}
$$

Let us assume $s=j \omega$, then there is a constant $\Lambda>0$ such that $|\Gamma(j \omega)|<\Lambda$, which is a superior bound to the factors as indicated in equation (4.25). As $[k / k]$ converges uniformly to $e^{-s \tau}$, then for each $\epsilon>0$ there is a $K$ such that for $k>K$, one has $\left|[k / k]-e^{-s \tau}\right|<\epsilon$, then, for each $\epsilon_{2}>0$ there is $K$ such that for $k>K$, one has:

$$
\left\|\Phi_{k}^{-1}(s)-\Phi^{-1}(s)\right\|_{\infty} \leq\left|[k / k]-e^{-s \tau}\right| \Lambda<\epsilon \Lambda=\epsilon_{2} .
$$

which concludes the proof.

Lemma 9. The sequence $\left\{\bar{\Lambda}_{22, k}(s)\right\}=C\left\{\Phi_{k}^{-1}(s)\right\} \bar{E}$ converge to $\bar{\Lambda}_{22, \text { delay }}=C\left(s I-\bar{A}_{0}-\right.$ $\left.F e^{-\tau s}\right)^{-1} \bar{E}$.

Proof. Since $\Phi_{k}^{-1}(s)$ is convergent almost everywhere in $\mathbb{C}$ and if it is just multiplied by constants on both sides, then the sequence continues to converge, and the lemma is proved.

Lemma 10. The sequence $\left\{\bar{\Lambda}_{22, k}(s)\right\}=C\left\{\Phi_{k}^{-1}(s)\right\} \bar{E}$ is uniformly convergent and tends to $\bar{\Lambda}_{22, \text { delay }}=C\left(s I-\bar{A}_{0}-F e^{-\tau s}\right)^{-1} \bar{E}$.

Proof. As this sequence is only the one in lemma 8 multiplied by two constants, the result follows. 
The following is an alternative definition (ZHOU; DOYLE; GLOVER, 1995) of structured singular value that is more suitable for the proof:

Definition 15. Given a matrix $M$ and a structured family of matrices $\Delta$ such that $\|\Delta\|_{\infty} \leq 1$ the structured singular value, also denoted by SSV or simply $\mu$, is a real non-negative function defined by :

$$
\mu_{\Delta}(M) \triangleq \frac{1}{\min _{\Delta}\{\bar{\sigma}(\Delta) \mid \operatorname{det}(I-M \Delta)=0\}}
$$

where $M$ is a complex matrix (matrix of transfer functions), $\Delta=\operatorname{diag}\left\{\Delta_{i}\right\}$ is a block diagonal matrix such that $\bar{\sigma}\left(\Delta_{i}\right) \leq 1$. Some of the blocks may be repeated and some may be restricted to be real. If no such structured $\Delta$ exists, then $\mu(M)=0$.

That is, what is being searched is $\Delta^{*}$, corresponding to the minimum of $\bar{\sigma}\left(\Delta^{*}\right)$ with the restriction $\operatorname{det}(I-M \Delta)=0$. It is therefore a optimization problem with restriction.

One would then have to define a sequence of functions given by:

$$
\mu_{k}(j \omega)=\left\{\mu_{\Delta}\left(\Lambda_{22, k}(j \omega)\right)\right\}
$$

that is, one would have to solve, for some values of $k$, an optimization problem with restriction for some values of $\omega \in R^{+}$. According to (DOYLE, 1982), those functions are continuous. Unfortunately, this type of optimization is complex to solve.

From (DOYLE, 1982), the optimization problem $\min _{D \in \mathcal{D}_{\Delta}} \bar{\sigma}\left(D M(j \omega) D^{-1}\right)$, where $\mathcal{D}_{\Delta}$ is the set of matrices that commute with $\Delta$, is convex, which means that there is also global minimum $D^{*}$. Then, let us define the following sequence:

Definition 16 (Upper Bound Sequence). The sequence of functions given by:

$$
m_{k}(j \omega)=\min _{D(\omega) \in \mathcal{D}} \bar{\sigma}\left(D(\omega) \Lambda_{22, k}(j \omega) D(\omega)^{-1}\right)
$$

where $D \in \mathcal{D}_{\Delta}$ is the set of matrices that commutes with $\Delta$, is the upper bound sequence for the sequence in (4.28).

To get conclusions about this sequence of functions, the following theorem is important:

Theorem 22. The complex function $m(j \omega)$ given by:

$$
m(j \omega)=\min _{D \in \mathcal{D}_{\Delta}} \bar{\sigma}\left(D G(j \omega) D^{-1}\right)=\min _{D \in \mathcal{D}_{\Delta}} \sqrt{\lambda_{\max }\left(D G(j \omega)\left(D^{\dagger} D\right)^{-1} G^{\dagger}(j \omega) D^{\dagger}\right)}
$$


that is, a function given by the optimization on one of the parameters, is continuous in $[0, \infty)$

Proof. Let's define the function $F(D, j \omega)=D G^{\dagger}(j \omega)\left(D^{\dagger} D\right)^{-1} G(j \omega) D^{\dagger}$ that is obviously continuous in $\omega$. As the eigenvalues are continuously dependent on the matrix entries, the function $\lambda_{\max }(F(D, j \omega))$ is also continuous in $\omega$ (see (MEYER, 2000) p. 497). Finally, as the square root is continuous for nonnegative values, $\bar{\sigma}(G(j \omega))=\sqrt{\lambda_{\max }(F(D, j \omega))}$ is continuous in $[0, \infty)$. Supposing that the optimization problem in $(4.30)$ is such that the set of decision values $\mathcal{G}(\omega)=K D_{\Delta}$ is a compact subset of $D_{\Delta}$ (which is reasonable to assume, as computers cannot store infinities) and the set valued function $\mathcal{G}(\omega)$ is also continuous (as it is constant), then applying the Theorem 26 (Berge's Continuous Maximum Theorem), the function $m(j \omega)$ must be continuous.

Corollary 1. The sequence of functions in (4.29) has only continuous functions in $[0, \infty)$.

Then, each function in the sequence presented in $(4.29)$ is continuous $(0, \infty)$. Now remains the task to prove that the sequence in (4.29) converges.

Theorem 23. The function of matrices :

$$
u(X)=\min _{D \in \mathcal{D}} \bar{\sigma}\left(D X D^{-1}\right)
$$

is continuous in the parameters $X$.

Proof. It is well known that $\bar{\sigma}\left(D X D^{-1}\right)=\sqrt{\lambda_{\max }\left(D X\left(D^{\dagger} D\right)^{-1} X^{\dagger} D^{\dagger}\right)}$ is a continuous function on $D$ and $X$, that is, the eigenvalues depend continuously on the matrix entries (see (MEYER, 2000) p. 497). Let us suppose that the values of $D$ used in the optimization belongs to a compact set for each possible $X$, which is reasonable, as the possible matrices $D$ represented in a computer should have limited values in their entries. Let us call this compact set $K D_{\Delta} \subset D_{\Delta}$. The set valued function $\mathcal{G}(X)=K D_{\Delta}$ is continuous, as it is constant. Then the set valued function $\mathcal{G}(X)$ is continuous and compact-valued. Then, by applying again the Berge's continuous maximum value theorem, the result follows.

Finally, one can prove that the sequence in (4.29) is convergent:

Theorem 24. The sequence (4.29) converges.

Proof. By the Lemma 9, the sequence $\left\{\bar{\Lambda}_{22, k}(s)\right\}$ converges. As $m_{k}(j \omega)=u\left(\bar{\Lambda}_{22, k}(j \omega)\right)$ and $u(X)$ is continuous in $X$, then the result follows. 
Theorem 21 can then be proved: the quantity $\mu\left(\Lambda_{22, \text { delay }}(j \omega)\right)$ is obviously well defined, as it represent the distance to lose absolute stability. If one guarantee that $m_{k}(j \omega)<1$ for every $k$, then $m_{\infty}(j \omega)<1$, which means that $\mu\left(\Lambda_{22 \text {,delay }}(j \omega)\right)<1$, and the absolute stability with delay is guaranteed.

On the other hand, it is impractical do verify $\mu\left(\Lambda_{22, k}(j \omega)\right)<1$ for each $k$. Then, if for a finite set of indexes $k_{1}, \cdots, k_{p}$ the condition is satisfied and there is a clear asymptotic behaviour of $m_{k}(j \omega)$ always $<1$, then one can use Padé approximation in order to verify that the system with pure delay is absolutely stable.

\subsection{Controller Synthesis}

The next theorem provides a necessary and sufficient condition for the robustness of stability and performance and it is an extension of Theorem 18. Based on this condition, it is possible via DK-Iteration (see section 2.7.5) to design a stabilizing controller that guarantees robustness of performance for Lurie type systems in closed-loop (Figure 21).

Theorem 25. Given a controller $K(s)$ and $\left[0, k_{j}\right]$ the sectors that include $f_{j} \in F_{\left(0, k_{j}\right]}$ for $j=1, \ldots, m$, then the system (2.28) with the controller $K(s)$ in feedback loop is robustly stable and have robustness of performance, if and only if:

$$
\mu_{\tilde{\Delta}}\left(\left[\begin{array}{cc}
M_{11}(s) & M_{12}(s) \\
M_{21}(s) & M_{22}(s)
\end{array}\right]\right)<1, \quad \forall \omega,
$$

where:

$$
\begin{gathered}
M_{11}(s)=I+C\left(s I-A_{0}\right)^{-1} B K(s)(-I)\left[I+C\left(s I-A_{0}\right)^{-1} B K(s)\right]^{-1}, \\
M_{12}=C\left(s I-A_{0}\right)^{-1} E+C\left(s I-A_{0}\right)^{-1} B K(s)(-C)\left(s I-A_{0}\right)^{-1} E\left[I+C\left(s I-A_{0}\right)^{-1} B K(s)\right]^{-1}, \\
M_{21}=C\left(s I-A_{0}\right)^{-1} E+C\left(s I-A_{0}\right)^{-1} B K(s)(-I)\left[I+C\left(s I-A_{0}\right)^{-1} B K(s)\right]^{-1}, \\
M_{22}=C\left(s I-A_{0}\right)^{-1} E+C\left(s I-A_{0}\right)^{-1} B K(s)-C\left(s I-A_{0}\right)^{-1} E\left[I+C\left(s I-A_{0}\right)^{-1} B K(s)\right]^{-1},
\end{gathered}
$$

with:

$$
A_{0}=\left[\begin{array}{ccc}
a_{11}-\sum_{j=1}^{m} b_{1 j} \frac{k_{j}}{2} c_{j 1} & \ldots & a_{1 n}-\sum_{j=1}^{m} b_{1 j} \frac{k_{j}}{2} c_{j n} \\
\vdots & \ddots & \vdots \\
a_{n 1}-\sum_{j=1}^{m} b_{n j} \frac{k_{j}}{2} c_{j 1} & \ldots & a_{n n}-\sum_{j=1}^{m} b_{n j} \frac{k_{j}}{2} c_{j n}
\end{array}\right]
$$

and:

$$
E=\left[\begin{array}{ccc}
-\frac{k_{1}}{2} b_{11} & \cdots & -\frac{k_{m}}{2} b_{1 m} \\
\vdots & \ddots & \vdots \\
-\frac{k_{1}}{2} b_{n 1} & \cdots & -\frac{k_{m}}{2} b_{n m}
\end{array}\right]
$$


Figure 57: $\Lambda-\Delta-\bar{\Delta}$-Structure in closed-loop with controller.

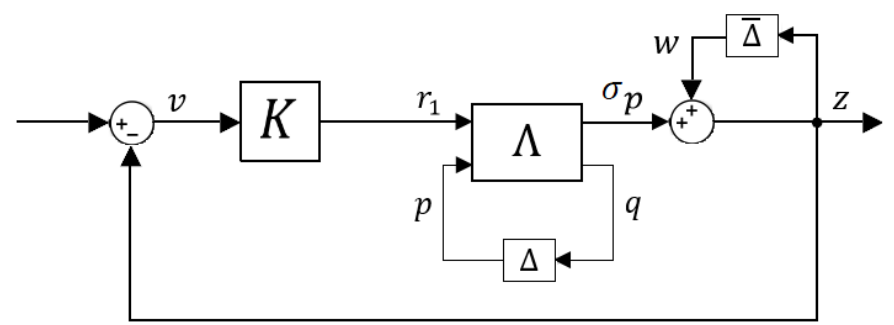

Source: Author.

Proof. Initially, from the system in Figure 57, one gets the matrix $M$ (see Figure 25). So, considering from the Theorem 18 the Laplace transforms:

$$
\begin{gathered}
x(s)=\left(s I-A_{0}\right)^{-1} B_{0} r_{1}+\left(s I-A_{0}\right)^{-1} E p, \\
\sigma(s)=C_{0}\left(s I-A_{0}\right)^{-1} B_{0} r_{1}+D_{0} r_{1}+F p+C_{0}\left(s I-A_{0}\right)^{-1} E p, \\
q(s)=G\left[\left(s I-A_{0}\right)^{-1} B_{0} r_{1}+\left(s I-A_{0}\right)^{-1} E p\right]+H r_{1},
\end{gathered}
$$

where:

$$
E=\left[E_{1}, \ldots, E_{m}\right], \quad F=\left[F_{1}, \ldots, F_{m}\right], \quad G=\left[G_{1}, \ldots, G_{m}\right]^{T}, \quad H=\left[H_{1}, \ldots, H_{m}\right]^{T},
$$

from Figure 57, one obtains $z=\sigma+w$, and $v=-\sigma-w$. Adopting $\left[r_{1}, w, p\right]^{T}$ as inputs and $[v, z, q]^{T}$ as outputs, one gets:

$$
\begin{gathered}
v(s)=-C_{0}\left(s I-A_{0}\right)^{-1} B_{0} r_{1}-D_{0} r_{1}+F p-C_{0}\left(s I-A_{0}\right)^{-1} E p-w \\
z(s)=C_{0}\left(s I-A_{0}\right)^{-1} B_{0} r_{1}+D_{0} r_{1}+F p+C_{0}\left(s I-A_{0}\right)^{-1} E p+w \\
q(s)=G\left[\left(s I-A_{0}\right)^{-1} B_{0} r_{1}+\left(s I-A_{0}\right)^{-1} E p\right]+H r_{1},
\end{gathered}
$$

in matrix form:

$$
\left[\begin{array}{l}
v \\
z \\
q
\end{array}\right]=\left[\begin{array}{ccc}
-C_{0}\left(s I-A_{0}\right)^{-1} B_{0}-D_{0} & -I & -C_{0}\left(s I-A_{0}\right)^{-1} E+F \\
C_{0}\left(s I-A_{0}\right)^{-1} B_{0}+D_{0} & I & C_{0}\left(s I-A_{0}\right)^{-1} E+F \\
G\left(s I-A_{0}\right)^{-1} B_{0}+H & 0 & G\left(s I-A_{0}\right)^{-1} E
\end{array}\right]\left[\begin{array}{c}
r_{1} \\
w \\
p
\end{array}\right]
$$

Thus, one obtains the general control system according to Figure 22, where $P(s)$ is the generalized plant. In this way, the previous system corresponds to: 


$$
\left[\begin{array}{l}
v \\
z \\
q
\end{array}\right]=\left[\begin{array}{lll}
P_{11}(s) & P_{12}(s) & P_{13}(s) \\
P_{21}(s) & P_{22}(s) & P_{23}(s) \\
P_{31}(s) & P_{32}(s) & P_{33}(s)
\end{array}\right]\left[\begin{array}{l}
r_{1} \\
w \\
p
\end{array}\right]
$$

Next, since one needs to find the M-structure, it is used an upper linear fractional transformation, i.e., $M=F_{U}(P, K)$ from (4.33), getting:

$$
\left[\begin{array}{l}
z \\
q
\end{array}\right]=\left[\begin{array}{cc}
P_{22}+P_{21} K P_{12}\left(I-P_{11} K\right)^{-1} & P_{23}+P 21 K P_{13}\left(I-P_{11} K\right)^{-1} \\
P_{23}+P_{31} K P 12\left(I-P_{11} K\right)^{-1} & P_{33}+P 31 K P_{13}\left(I-P_{11} K\right)^{-1}
\end{array}\right]\left[\begin{array}{c}
w \\
p
\end{array}\right] .
$$

From (4.34) one extracts $M$ according to (2.67), and Figure 25, as follows:

$$
\left[\begin{array}{ll}
M_{11} & M_{12} \\
M_{21} & M_{22}
\end{array}\right]=\left[\begin{array}{cc}
P_{22}+P_{21} K P_{12}\left(I-P_{11} K\right)^{-1} & P_{23}+P_{21} K P_{13}\left(I-P_{11} K\right)^{-1} \\
P_{23}+P_{31} K P_{12}\left(I-P_{11} K\right)^{-1} & P_{33}+P_{31} K P_{13}\left(I-P_{11} K\right)^{-1}
\end{array}\right]
$$

By Lemma 4:

$$
A_{0}=\left[\begin{array}{ccc}
a_{11}-\sum_{j=1}^{m} b_{1 j} \frac{k_{j}}{2} c_{j 1} & \ldots & a_{1 n}-\sum_{j=1}^{m} b_{1 j} \frac{k_{j}}{2} c_{j n} \\
\vdots & \ddots & \vdots \\
a_{n 1}-\sum_{j=1}^{m} b_{n j} \frac{k_{j}}{2} c_{j 1} & \ldots & a_{n n}-\sum_{j=1}^{m} b_{n j} \frac{k_{j}}{2} c_{j n}
\end{array}\right]
$$

$B_{0}=B, C_{0}=C, D_{0}=0, E_{j}=\left[\begin{array}{c}-\frac{k_{j}}{2} b_{1 j} \\ \vdots \\ -\frac{k_{j}}{2} b_{n j}\end{array}\right] \rightarrow E=\left[E_{1}, \ldots, E_{m}\right], F_{j}=0, G_{j}=\left[\begin{array}{l}c_{j 1} \\ \vdots \\ c_{j n}\end{array}\right]^{T}$ $\rightarrow G=C, H_{j}=0$, for $j=1, \ldots, m$.

So:

$$
M_{11}(s)=I+C\left(s I-A_{0}\right)^{-1} B K(s)(-I)\left[I+C\left(s I-A_{0}\right)^{-1} B K(s)\right]^{-1},
$$$$
M_{12}=C\left(s I-A_{0}\right)^{-1} E+C\left(s I-A_{0}\right)^{-1} B K(s)(-C)\left(s I-A_{0}\right)^{-1} E\left[I+C\left(s I-A_{0}\right)^{-1} B K(s)\right]^{-1},
$$$$
M_{21}=C\left(s I-A_{0}\right)^{-1} E+C\left(s I-A_{0}\right)^{-1} B K(s)(-I)\left[I+C\left(s I-A_{0}\right)^{-1} B K(s)\right]^{-1},
$$$$
M_{22}=C\left(s I-A_{0}\right)^{-1} E+C\left(s I-A_{0}\right)^{-1} B K(s)-C\left(s I-A_{0}\right)^{-1} E\left[I+C\left(s I-A_{0}\right)^{-1} B K(s)\right]^{-1} .
$$

By the condition of (2.75), the theorem is proved.

Remark 18. By Theorem 18, one has that the previous theorem will guarantee the absolute stability to a Lurie type system with a controller $K(s)$ if the condition in (4.31) is satisfied. 
Remark 19. A similar theorem can be constructed, without much difficulty, that allows a design that guarantees only robust stability. Note that if the system has robustness of performance, then it will be robustly stable (SKOGESTAD; POSTLETHWAITE, 200\%). The difference is that if one designs only a stabilizing controller, maybe it will be less conservative.

Remark 20. In a similar way, as was done in the Theorem 19, a theorem can be easily obtained for controller synthesis with nonlinearities of class $F_{\left[\underline{k}_{j}, \bar{k}_{j}\right]}$.

Example 8. Revisiting the Example 6, one wants to design controllers that stabilize the neural network and have robustness of performance for two cases: a) system without time-delay (Figure 58); and b) system with time-delay (Figure 59).

Figure 58: Neural network without time-delay.

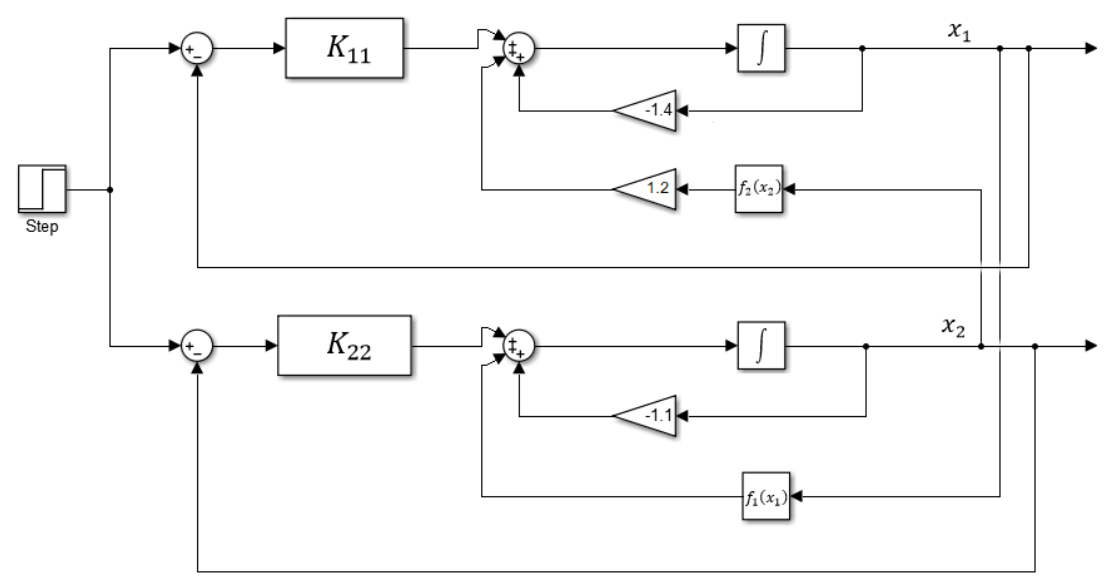

Source: Author.

Figure 59: Neural network with time-delay.

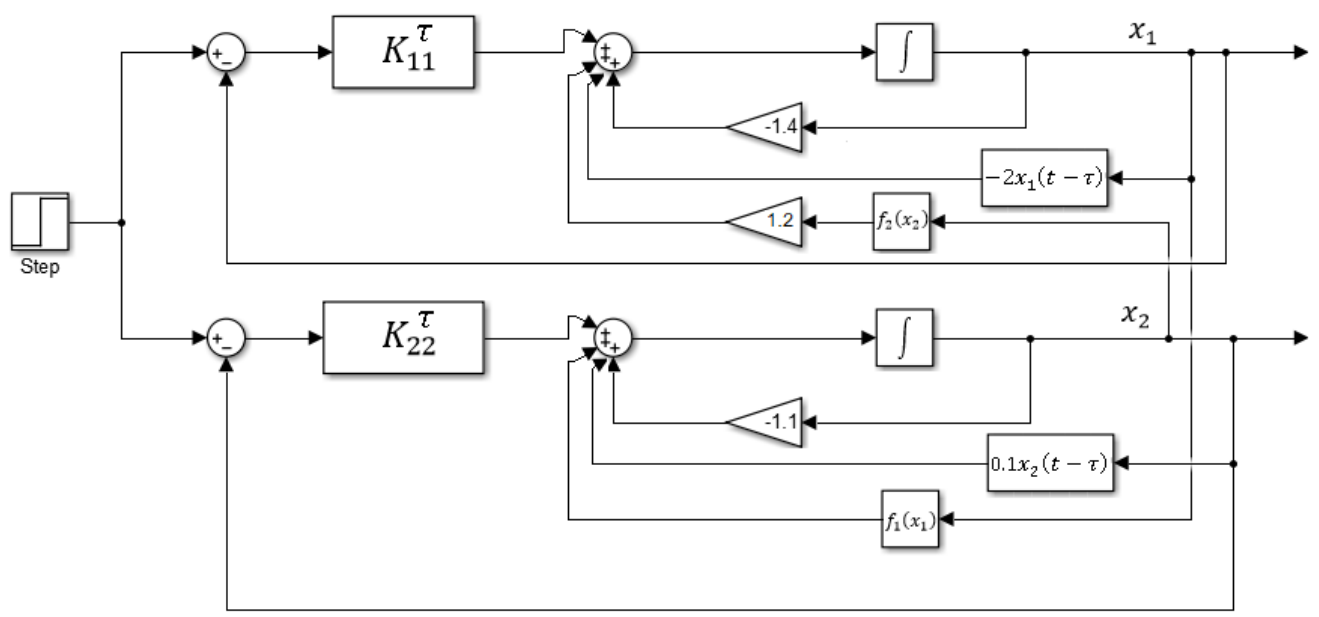

Source: Author. 
Figure 60: $\mu$-analysis of stability.

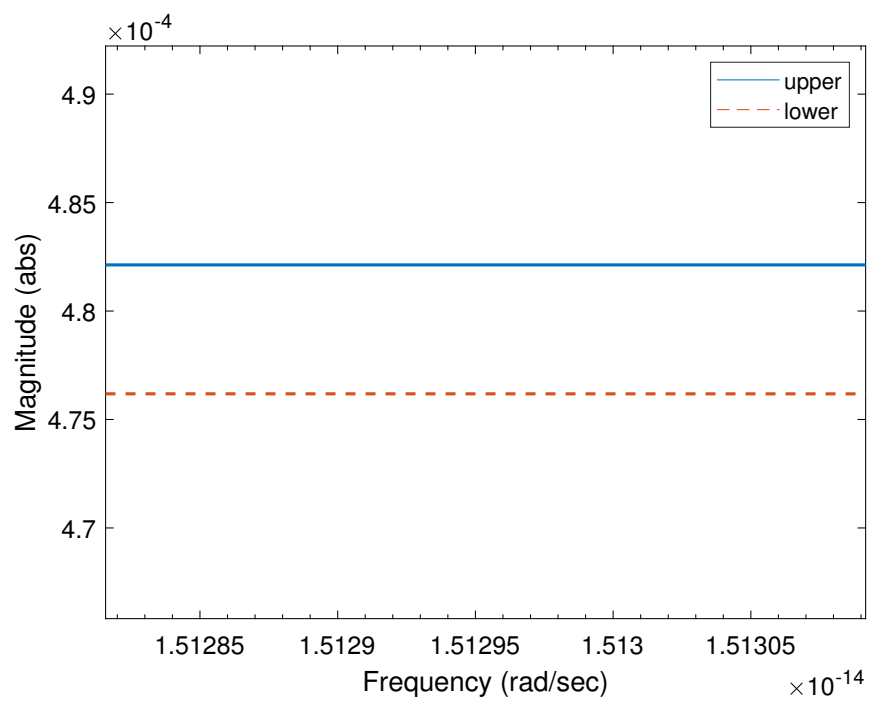

Source: Author.

a) In order to obtain the controller for the system without time-delay, one uses DKIteration as explained in section 2.7.5, and the conditions of Theorem 25. From this theorem, one gets matrices $P(s)$ and $M(s)$. Note that the term $\left(s I-A_{0}\right)$ is part of these matrices for systems without time-delay. In this case, the structure block is BlockStrucure $1=$ $\left[\begin{array}{lll}-1 & 0 ;-1 & 0\end{array}\right]$ for stability analysis and BlockStrucure $2=\left[\begin{array}{llll}-1 & 0 ;-1 & 0 ; 2 & 2\end{array}\right]$ for performance analysis. It is known that the condition of the robustness of performance via $H_{\infty}$-Norm is $\left\|F_{L}(M, \Delta)\right\|_{\infty}<1$. In the first iteration, the norm value is 1.4144 . Using DK-Iteration, after three iterations, one gets $\left\|F_{L}(M, \Delta)\right\|_{\infty}=1.0067$. The controller obtained is then:

$$
K=\left[\begin{array}{ll}
K_{11} & K_{12} \\
K_{21} & K_{22}
\end{array}\right]=\left[\begin{array}{cc}
\frac{49480 s+4948}{s+0.07763} & 0 \\
0 & \frac{45490 s+9098}{s+0.1767}
\end{array}\right]
$$

Using this controller, the $m(j \omega)$ of robustness of stability is 0.000019 (see Figure 60). This means that the controller stabilizes the nonlinear system. Regarding performance analysis, the $m(j \omega)$ is 1 . This value meets the performance specification for practically the whole set of uncertainties, since it is equal to 1 (SKOGESTAD; POSTLETHWAITE, 2007). In order to validate this result, a simulations of the system in Figure 58 with nonlinearity $f_{i}\left(x_{i}\right)=\frac{2}{\pi} \arctan \left(1.4 \frac{\pi}{2} x_{i}\right), i=1,2$, and unit step input is conducted. One gets in Figure 61 a time response without controller and one observes that the system has not a good performance and has steady state error. Inserting the controller $K(s)$, one observes in Figure 62 that the system has excellent performance and presents no error. 
Figure 61: Temporal response without control.

Figure 62: Temporal response with control.

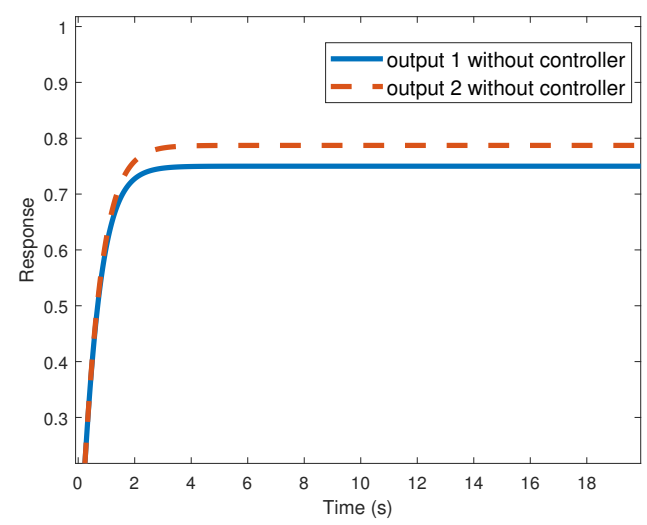

Source: Author.

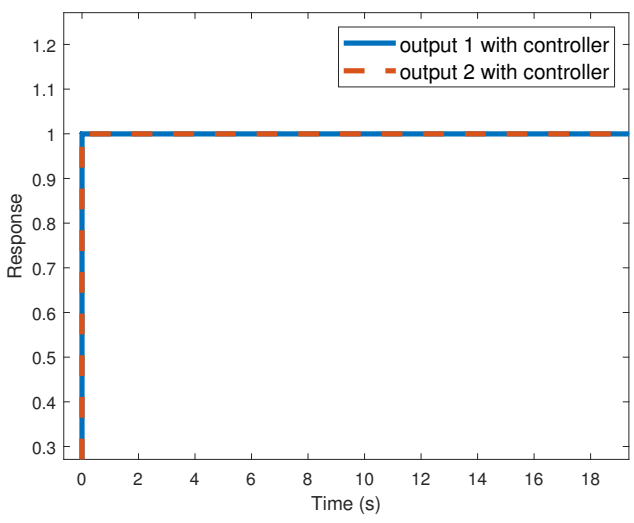

Source: Author.

b) In order to obtain the controller for system with time-delay, the procedures are analogous to item a), with one difference: to construct matrices $P(s)$ and $M(s)$, one uses the term $\left(s I-\bar{A}_{0}-F e^{-\tau s}\right)^{-1}$ from the Theorem 21 with Padé approximation of order 3. In this example, the matrix $F$ of (4.7) is $\left(\begin{array}{cc}-2 & 0 \\ 0 & 0.1\end{array}\right)$. In Figure 63, one has the system without controller and after one iteration one gets satisfactory results, according to the Figure 64.

Figure 63: Time-delayed without control. $\quad$ Figure 64: Time-delayed with control.

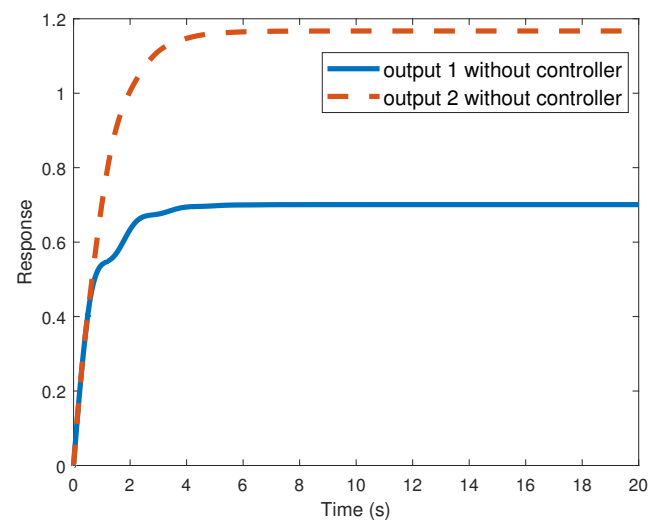

Source: Author.

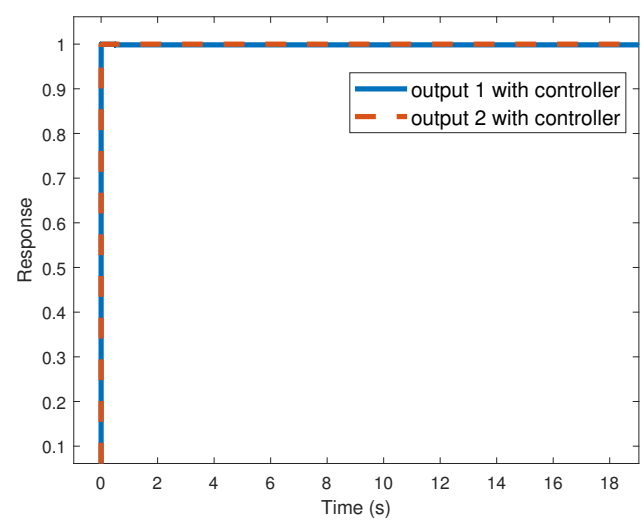

Source: Author.

Remark 21. In the above example, it is applied this method to design controllers for a complex network without delay between neurons. If one wants to include time-delay between nodes, one would have to consider systems like: 


$$
\left\{\begin{array}{l}
\dot{x}=A x-B_{1} f_{1}\left(\sigma_{1}\right)-B_{2} f_{2}\left(\sigma_{2}\right)+F x(t-\tau)+B r_{1}, \\
\sigma_{1}=C_{1} x \\
\sigma_{2}=C_{2} x(t-\tau)
\end{array}\right.
$$

where $x \in \mathbb{R}^{n}$ is the state vector, $\tau>0$ is a real constant delay, $f_{i}(\sigma) \in \mathbb{R}^{m}$ and $\sigma_{i} \in \mathbb{R}^{m}$. One has the fixed matrices $F \in \mathbb{R}^{n \times m}, B_{i} \in \mathbb{R}^{n \times m}, C_{i} \in \mathbb{R}^{m \times n}$ and $A \in \mathbb{R}^{n \times n}$ is Hurwitz. Such class of systems has time-delay in the output, which include distributed control and distributed filtering (DING; WANG; HAN, 2020). The Theorem 21 could be easily generalized to this case by applying the Laplace transform in (4.36). In fact, the matrix $F$ in (4.36) would become uncertain and one would have to define a matrix $\bar{F}$ similar to matrices $\bar{A}_{0}$ and $\bar{E}$ in the theorem. 


\section{APPLICATION}

In a woman of 51 years old, Alois Alzheimer (ALZHEIMER, 1907) described clinical observations, whose anatomical features were unusual to any previously known disease. The first symptom, which the woman demonstrated, was the idea of being jealous of her husband, and in a short time, she developed a rapid memory loss. This terrible disease became known by name of its discoverer. It is a degenerative disease that leads to death, and one of its main consequences is the loss of memory due to synaptic losses (TERRY, 2000). Although it has been documented more than a hundred years ago, the understanding of its final cause still represents a mystery to neuroscience.

On the other hand, and linked to neuroscience, the artificial neural networks (ANN) are computational methods inspired in the functioning of biological neurons, which has been widely used in several areas, including medicine (GIL et al., 2009; AL-SHAYEA, 2011). In particular, one has Hopfield neural network (HNN), proposed by (HOPFIELD, 1984). HNN is a kind of complex networks as cited in (BOCCALETTI et al., 2006) and can be used to simulate associative memory. Those networks have the ability to learn some behaviors that are presented to them in a process called training. HNN has been applied considerably in several areas, like optimization problems (KASZKUREWICZ; BHAYA, 1995) and other applications such as presented in (BRAGA; CARVALHO; LUDEMIR, 2005): implementation of an identification systems of military target used in aircraft model B-52; user authentication systems; oil exploration; prediction in the financial market. Currently the network has awakened great interest by theoretical, biological (MONTEIRO, 2006), because they can simulate self-associative memory as cited by (IYERNGAR; BALAGANI, 2004) and can be used in simulations of stages of Alzheimer's disease (AD).

One can ask: what do these two areas have in common with LP and the sufficient conditions presented in this work? Well, although LP has several applications, as listed in the introduction, this section is dedicated to an original application. Here, Alzheimer's disease, Hopfield's networks, and Lurie problem are related. 


\subsection{Alzheimer's Disease}

$\mathrm{AD}$ is a degenerative brain disease that deteriorates cognitive abilities and motor functions. It was first described in a 51-year-old woman by German psychiatrist and pathologist Alois Alzheimer (ALZHEIMER, 1907). Its causes are uncertain and unreliable, being its method of diagnosis made through the patient's medical history, neurological, psychiatric, clinical examinations, neuropsychological tests, and laboratory studies. For a definite diagnosis, it is necessary to deepen histopathology (MCKHANN et al., 1984), that is, an analysis of all the composition of brain tissue, which is not possible with the patient in life. Its symptoms involve memory loss (TERRY, 2000), progressive dementia, agitation, apathy, depression, loss of appetite, muscle contractions and others. There is no cure for $\mathrm{AD}$, however there are medications to slow down and relieve the symptoms (FILLIT; CUMMINGS, 2000; HAKE, 2002).

The classical neuropathological marks of AD involve characteristics of macroscopic and microscopic dimensions. These markers are useful for the diagnosis of the disease. In general, the macroscopic aspects are: $15 \%$ to $35 \%$ brain weight reduction, cortex atrophy, ventricular dilation, reduced hippocampus and decreased blood flow (FREITAS, 2006). The microscopic aspects in general are: Synaptic loss, neuronal death, amyloid plaque findings and neurofibrillar entanglements (SELKOE, 2001; FREITAS, 2006).

Neurons communicate with each other at contact points called synapses. In a synapse, a neuron sends a message to a target neuron. Synaptic loss is responsible for cognitive decline. It does not come only from the loss of neurons, so much so that it is possible in a specific region to have fewer synaptic connections than neurons. This fact indicates that synaptic loss precedes neuronal loss and, consequently, remaining neurons have low synaptic connections between their partners. For this reason, synaptic density is considered one of the main cognitive declines in AD (SCHEFF et al., 2007).

\subsection{Hopfield's Network and its Relationship with Lurie Problem}

HNN was proposed by Hopfield (HOPFIELD, 1984) and is part of an area known as neurodynamics. It can be represented by the following system of nonlinear differential equations:

$$
C_{i} \frac{d u_{i}}{d t}=-\frac{u_{i}}{R_{i}}+\sum_{j=1}^{n} T_{i j} V_{j}+I_{i}, \quad i=1,2, \ldots, n
$$


where $C \in \mathbb{R}^{n}, u \in \mathbb{R}^{n}, R \in \mathbb{R}^{n}, T \in \mathbb{R}^{n x n}, I \in \mathbb{R}^{n}$, and $V_{j}=g\left(u_{i}\right)$. The functions $g$ can be defined as $g: \mathbb{R} \rightarrow[0,1]$ and is continuously differentiable and monotonically increasing, or $g_{i}^{\prime}\left(u_{i}\right)>0$. This functions are called activation functions and usually are sigmoidal functions. The continuous model of HNN can be thought as an electric circuit (for more details (PINHEIRO, 2015)).

The problem of stability of HNN was initially discussed by (HOPFIELD, 1984). He first defined a function which he called as computational power function:

$$
E(V)=-\frac{1}{2} \sum_{i=1}^{n} \sum_{j=1}^{n} T_{i j} V_{i} V_{j}-\sum_{i=1}^{n} V_{i} I_{i}+\sum_{i=1}^{n} \frac{1}{R_{i}} \int_{0}^{V_{i}} g_{i}^{-1}(s) d s .
$$

Then, he showed that $\frac{d E}{d t}<0$ if the weight matrix $T$ is symmetric and if no neuron feeds itself back, thus concluding the stability of the network (see Figure 65).

Figure 65: Hopfield's stability.



Source: Author.

In (LIAO; YU, 2008) and (PINHEIRO; COLÓN, 2019) it is shown that HNN can be considered a special case of MIMO Lurie type system. Thus, considering the equation (5.1) after some changes of variables, it can take the following form:

$$
\frac{d x_{i}}{d t}=-d_{i} x_{i}+\sum_{j=1}^{n} b_{i j} f_{j}\left(x_{j}\right)
$$

In relation to the function $f$, one has $f \in F_{(0, k]}$. Comparing the equations in (5.3) with the equations in (1.4):

$$
\dot{y}_{i}=\sum_{j=1}^{n} \tilde{a}_{i j} y_{j}+\sum_{j=n-m+1}^{n} \tilde{b}_{i j} \tilde{f}_{j}\left(y_{j}\right),
$$

one has that the HNN is a special case of a MIMO Lurie type, where $\tilde{a}_{i j}=0, i \neq j$, $\tilde{a}_{i i}=-d_{i}<0$, e $m=n$. 


\subsection{Modeling Memory Loss Due to Alzheimer-Like's Disease via Hopfield Networks}

In this work, one of the consequences of Alzheimer's disease is modeled: memory loss. In order to do this, it is computationally modeled via a HNN the decrease of synapse strength between the neurons, because this is one of the causes of memory loss in Alzheimer's disease, as seen in the section 5.1. The designation Alzheimer-like was put because the artificial network presents only a consequence of the Alzheimer's disease, namely memory loss due to loss or weakening of synapses.

From (5.1), in the equation (5.4) is established a neural network conceiving a system of ordinary differential equations of 12 th order, that is, 12 Hopfield's neurons with architecture according to Figure 66 , which is represented visually by a $3 \times 4$ digital sign.

$$
\dot{u}_{i}=-d_{i} u_{i}+T_{i, n-(i-1)} \tanh \left(u_{n-(i-1)}\right)+I_{i}
$$

Figure 66: Network architecture to simulate a $3 \times 4$ digital sign.

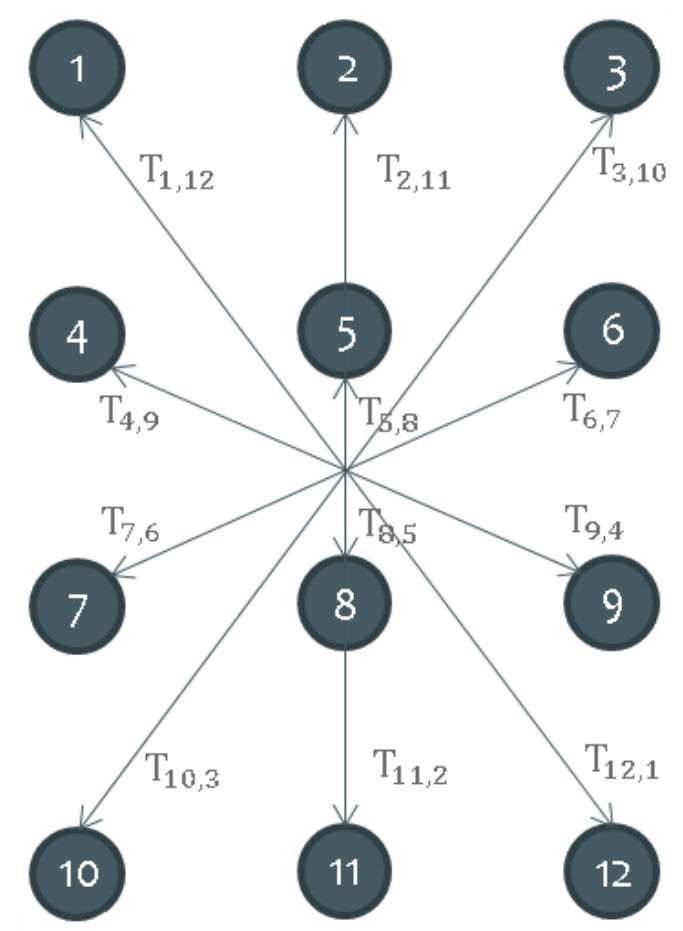

Source: Author.

In (ZHOU et al., 2016) it is suggested how to treat the components $I$ and $u$ of the equation (5.4). The entries $I$ (bias) is the pattern to be memorized, given by the vector $I=\left[I_{1}, I_{2}, I_{3}, I_{4}, I_{5}, I_{6}, I_{7}, I_{8}, I_{9}, I_{10}, I_{11}, I_{12}\right]$. For example, for the network to memorize the letter $\mathrm{L}$, the following vector must be provided (note that 1 represents black and -1 
represents white):

$$
I=[1,-1,-1,1,-1,-1,1,-1,-1,1,1,1] \cong\left[\begin{array}{ccc}
1 & -1 & -1 \\
1 & -1 & -1 \\
1 & -1 & -1 \\
1 & 1 & 1
\end{array}\right] .
$$

The initial conditions of the $u$ states are patterns to be recovered, given by the vector $u^{0}=\left[u_{1}^{0}, u_{2}^{0}, u_{3}^{0}, u_{4}^{0}, u_{5}^{0}, u_{6}^{0}, u_{7}^{0}, u_{8}^{0}, u_{9}^{0}, u_{10}^{0}, u_{11}^{0}, u_{12}^{0}\right]$. The vector $d$ is composed of fixed positive constants, because it is a condition to be Hopfield network. We chose $d=1$ for simplicity. In order to have a stable network the $T$ matrix must be symmetrical. One uses a matrix of $T_{12 \times 12}$ symmetrical with main diagonal zero. One can not say how a real biological neural network is connected. It is chosen $T_{0}$ like in equation (5.6) for simplicity. It will be seen that values around $0.005 T_{0}$ represents healthy neurons, and values around $0.6 T_{0}$ represents irreversible memory loss. The stability condition of the network and the correct adjustment of the weights ensure healthy memory. The network being stable, i.e., the matrix of weights being symmetric and without feedback of a neuron itself, ensures that, given an initial condition, the network finds an equilibrium point. This is a characteristic of the HNN, it is a finder of equilibrium points, as can be seen in the Figure 65.

Given an initial condition, for the network to find the correct equilibrium point (the memorized pattern given by the vector $I$ ), it is necessary to adjust the weights. In the section 5.1, it is seen that cognitive impairment in patients with Alzheimer's disease is associated with loss of synapses. These synaptic losses (see Figure 67) are related to the disturbances in the weights of the Hopfield's network (see Figure 68). In this case, the values $T_{i j}$ can be considered the synaptic connection strength.

Figure 67: Synapse between neurons.

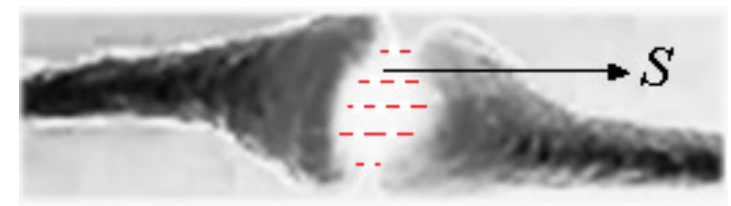

Source: Author. 
Figure 68: Hopfield's network via Simulink ${ }^{\circledR}$.

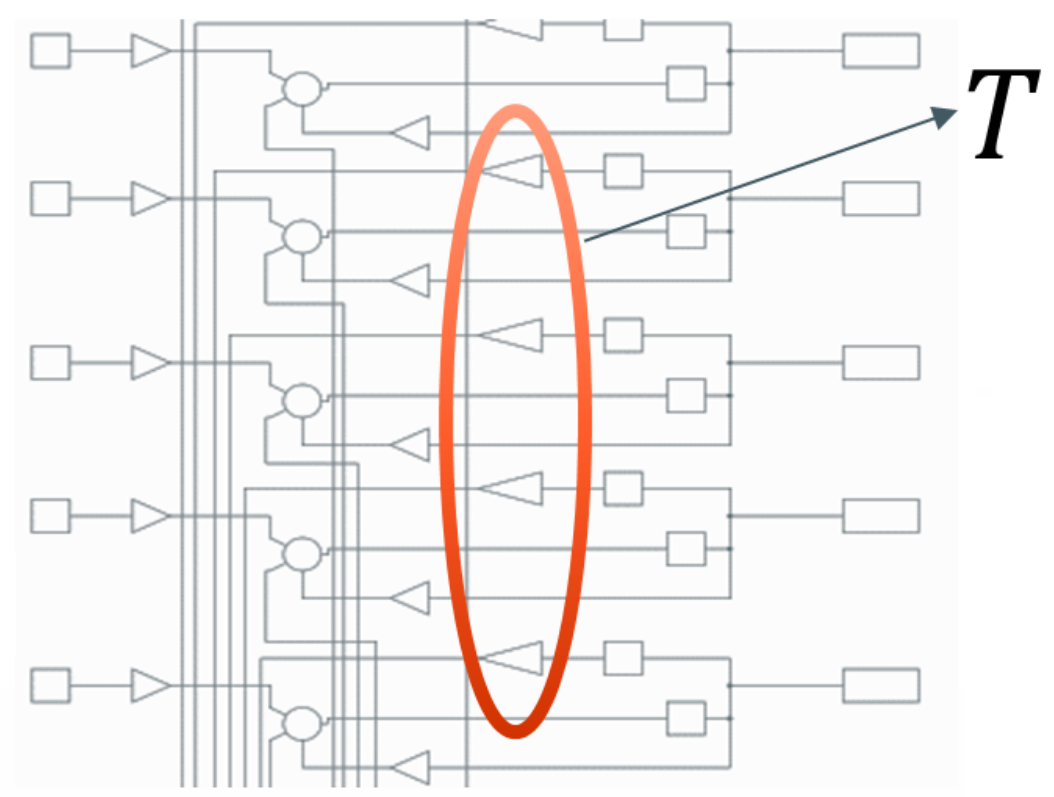

Source: Author.

Therefore, in our model, the lower the value of $T_{i j}$ the greater the connection strength $(S)$ between the neurons. In this situation one has a healthy memory. And the higher the value of $T_{i j}$ the lower is the connection strength $(S)$ between the neurons, which starts to remit in synaptic losses, leading to memory loss, characterizing Alzheimer's disease. The box in Figure 69 summarizes the process of modeling memory loss.

Figure 69: Memory loss modeling process.

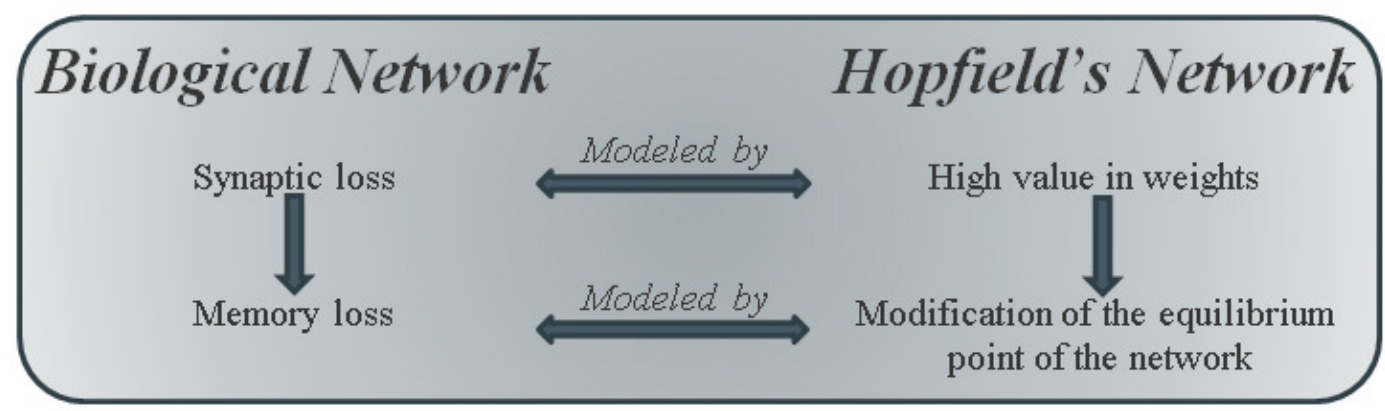

Source: Author.

\subsubsection{Simulation}

The purpose of the simulation is to show the functioning of the Hopfield's network as a healthy memory and then as a pathology (Alzheimer's disease). The selected input is the letter $\mathrm{L}$ as (5.5), and it is used the initial condition $u^{0}=[1,-1,-1,1,-1,-1,1,-1,-1,1,1,1]$. 
Healthy Network: In order to have a healthy network, it is inserted the matrix of weights $T=0.005 T_{0}$, where $T_{0}$ is as in (5.6). Note that the weight matrix $T$ is symmetrical and no neuron feeds itself back, and this guarantees stability for the network. Now observing the 0.005 value, this constant makes the weights small which leads the network to take as equilibrium points the $I$ input as can be observed in the temporal response of Figure 70 .

$$
T_{0}=\left[\begin{array}{cccccccccccc}
0 & 0 & 0 & 0 & 0 & 0 & 0 & 0 & 0 & 0 & 0 & 1.5 \\
0 & 0 & 0 & 0 & 0 & 0 & 0 & 0 & 0 & 0 & 1.5 & 0 \\
0 & 0 & 0 & 0 & 0 & 0 & 0 & 0 & 0 & 1.5 & 0 & 0 \\
0 & 0 & 0 & 0 & 0 & 0 & 0 & 0 & 1.5 & 0 & 0 & 0 \\
0 & 0 & 0 & 0 & 0 & 0 & 0 & 1.5 & 0 & 0 & 0 & 0 \\
0 & 0 & 0 & 0 & 0 & 0 & 1.5 & 0 & 0 & 0 & 0 & 0 \\
0 & 0 & 0 & 0 & 0 & 1.5 & 0 & 0 & 0 & 0 & 0 & 0 \\
0 & 0 & 0 & 0 & 1.5 & 0 & 0 & 0 & 0 & 0 & 0 & 0 \\
0 & 0 & 0 & 1.5 & 0 & 0 & 0 & 0 & 0 & 0 & 0 & 0 \\
0 & 0 & 1.5 & 0 & 0 & 0 & 0 & 0 & 0 & 0 & 0 & 0 \\
0 & 1.5 & 0 & 0 & 0 & 0 & 0 & 0 & 0 & 0 & 0 & 0 \\
1.5 & 0 & 0 & 0 & 0 & 0 & 0 & 0 & 0 & 0 & 0 & 0
\end{array}\right]
$$

Figure 70: Healthy network temporal response.

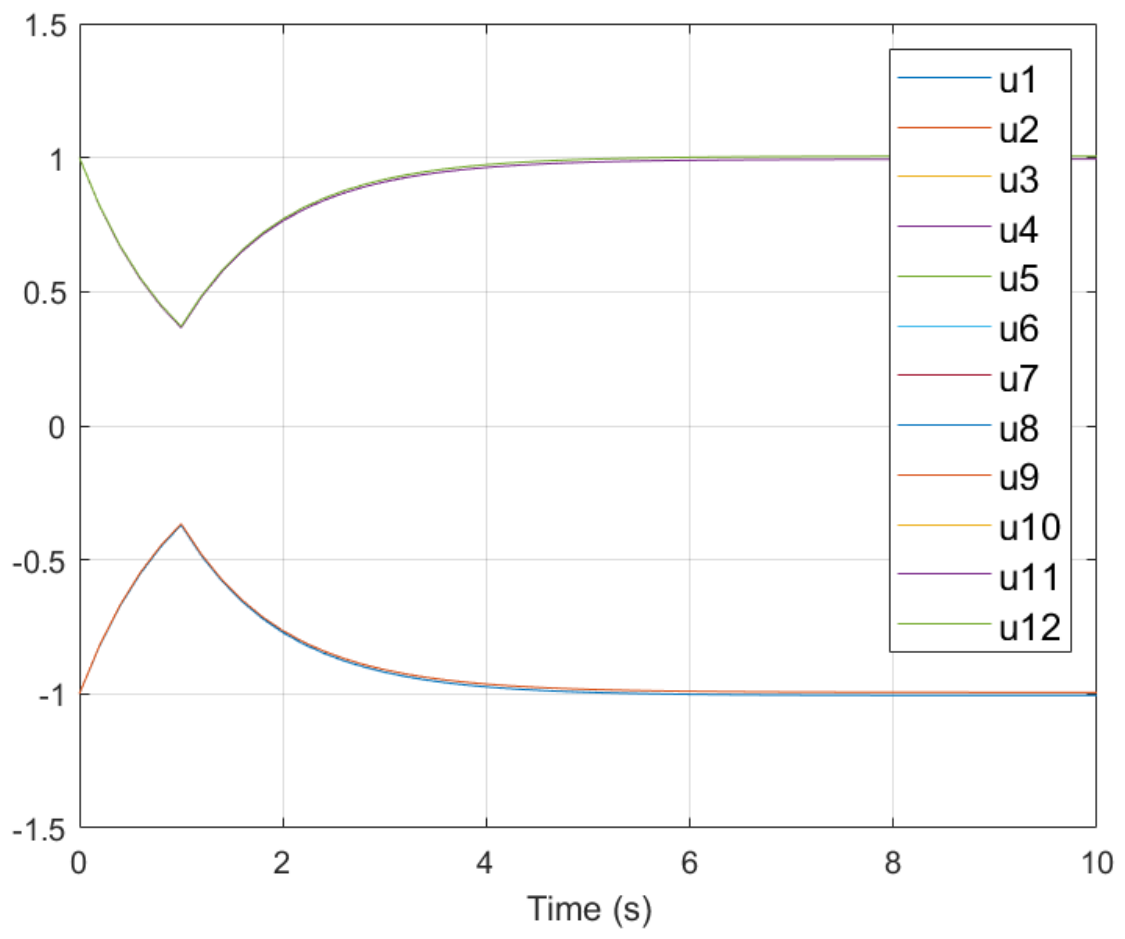

Source: Author. 
Therefore, one has a healthy memory, that is, the network is able to remember the letter L. The Figure 71 shows via a digital sign the evolution of the network in the process of remembering the letter L.

Figure 71: Temporal evolution of the memory of the letter L via digital sign.
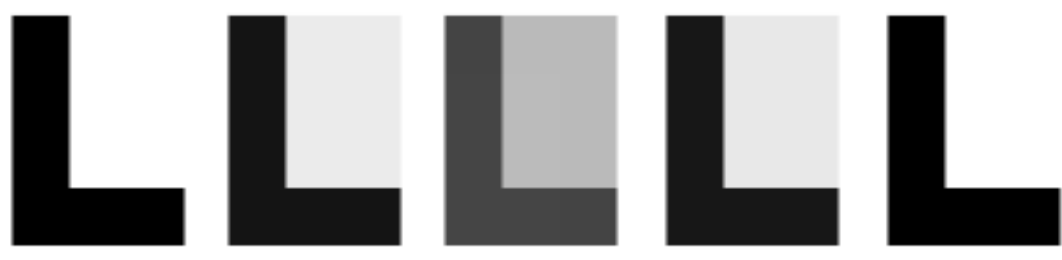

Source: Author.

\section{Network with Alzheimer-Like's Disease:}

First, it is simulated the weakening of the synaptic connection between the neurons. It is increased the value of the weight by 100 times, i.e., instead of multiplying the $T_{0}$ matrix, in (5.6) by 0.005 , it is multiplied by 0.5 , i.e., $T=0.5 T_{0}$. In the network equilibrium points change, according to the temporal response of Figure 72, models memory loss in Alzheimer's disease. Note that the network remains stable, but now with other equilibrium points. In the representation by the digital sign in Figure 73, one can see that the network looses the ability to remember the letter L correctly.

Figure 72: Temporal response for network with partial memory loss.

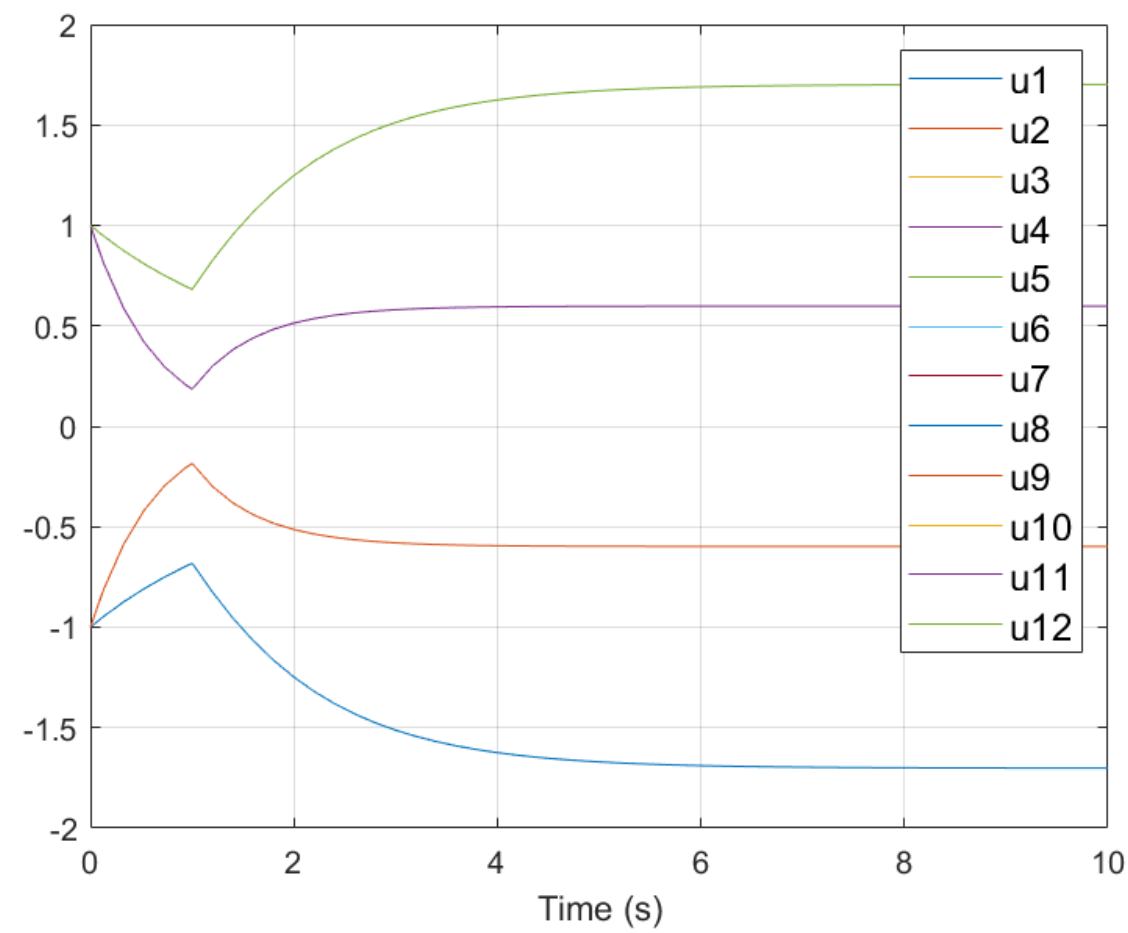

Source: Author. 
Figure 73: Temporal evolution of the letter L for network with partial loss of memory via digital sign.
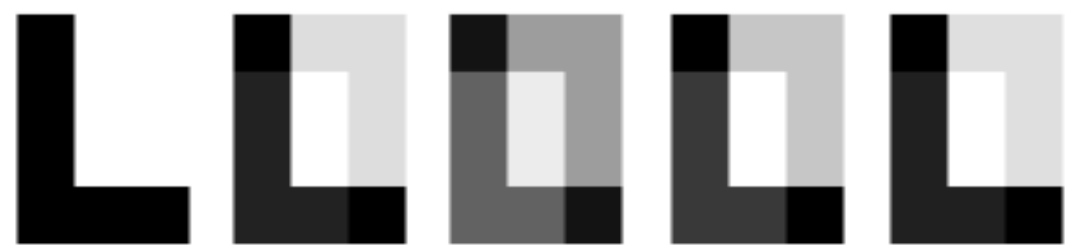

Source: Author.

Finally, it is taken $T=200 T_{0}$. Note in Figure 74 that the network assumes new equilibrium points, and by the digital sign of Figure 75, it completely looses its memory, not being able to remember the letter $\mathrm{L}$.

Figure 74: Temporal response of the worst case of memory loss.

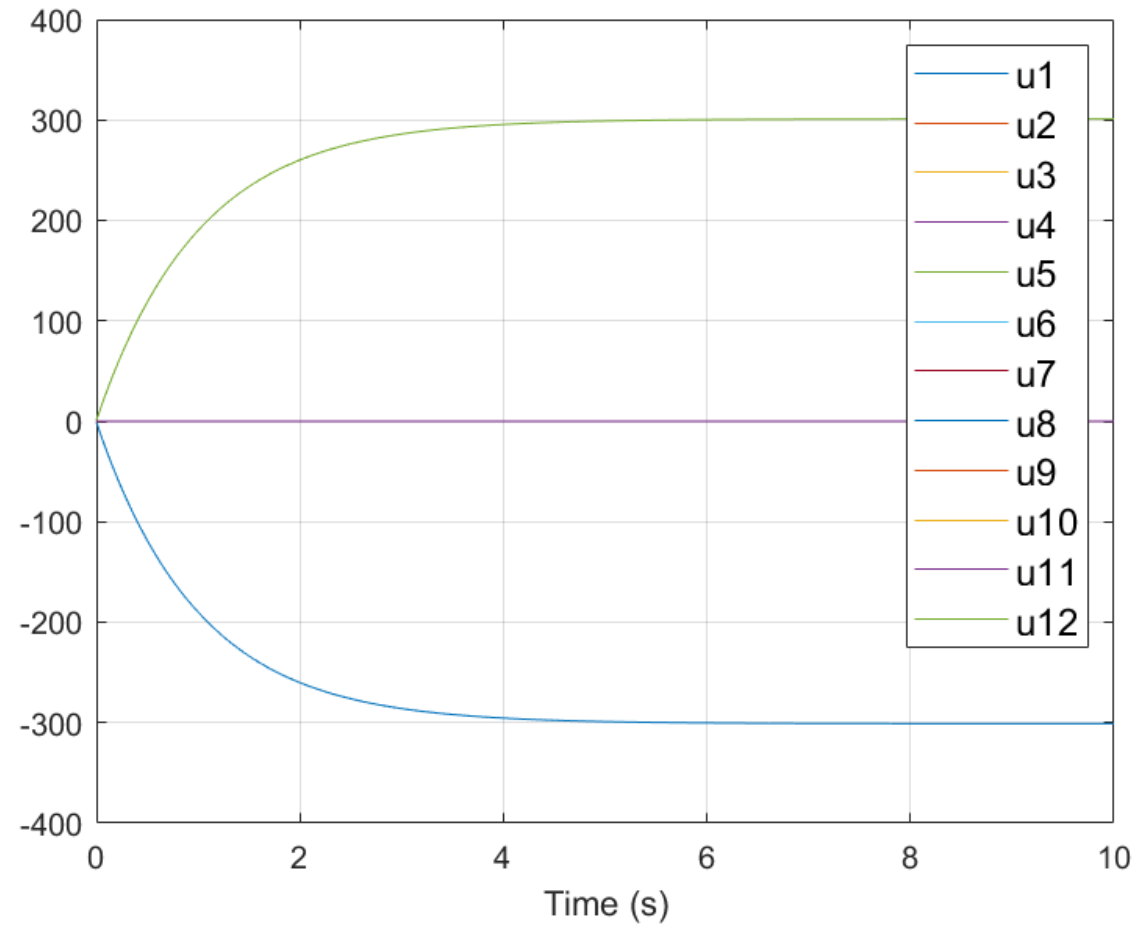

Source: Author.

\subsection{Application of a Result of Lurie Problem in Hop- field's Network}

In this section, an application of the Theorem 25 is made. This theorem together with $\mu$-synthesis (via DK-iteration), provide mechanisms for the design of a controller that ensures robustness of stability and performance for the network in (5.4). The goal is to correct the memory failure in the network, thus simulating a kind of cure in the 
Figure 75: Complete loss of memory via digital sign.
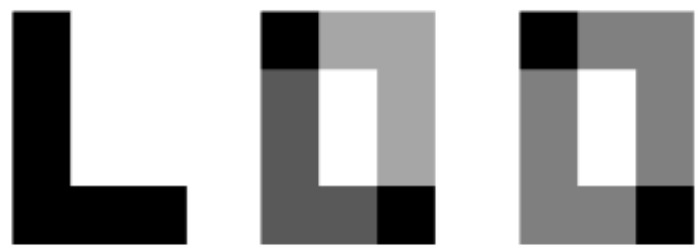

Source: Author.

computationally modeled disease. The Algorithm 1, brings the procedures and Matlab ${ }^{\circledR}$ function suggested to perform the DK-iteration. Note that the algorithm provides a matrix of controllers $K=\operatorname{diag}\left[K_{1}(s), \ldots, K_{12}(s)\right]$, which must be placed in the network according to Figure 76. In comparison with the network in Figure 68, a feedback loop (in red) is created.

Figure 76: Hopfield's network with controllers.

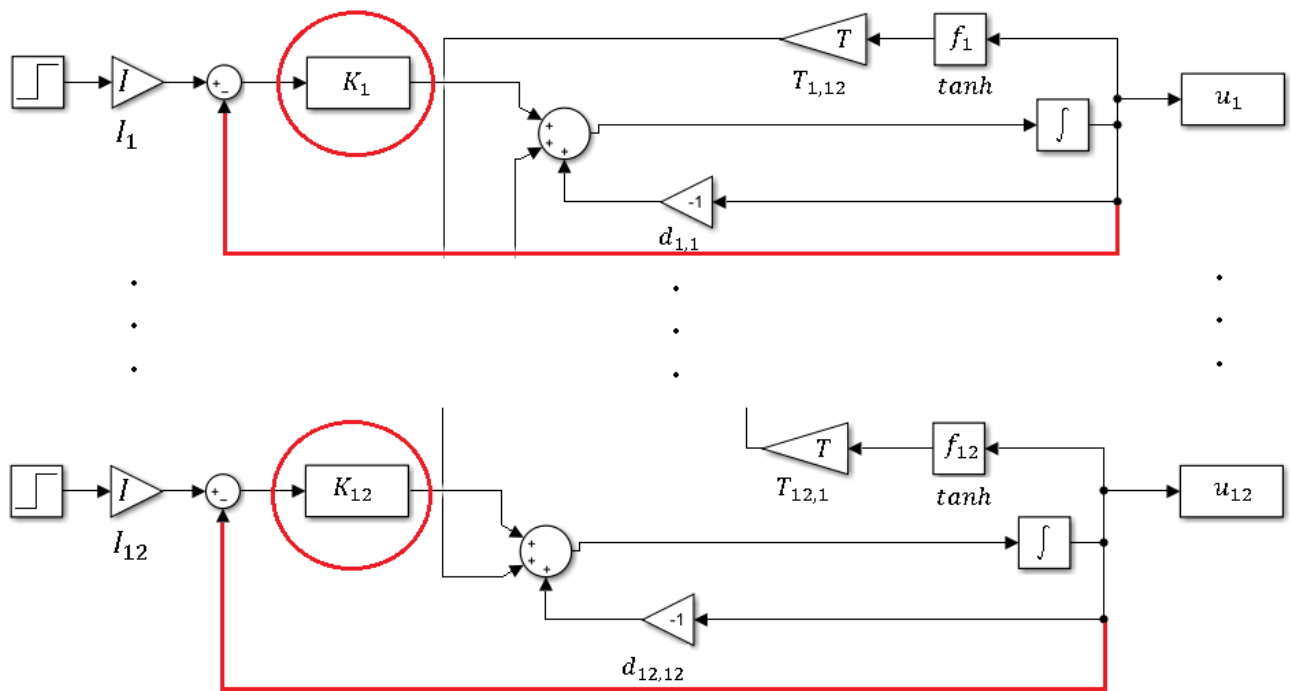

Source: Author.

\subsubsection{Simulation}

In this simulation, the problem of memory failure in the network presented by Figure 72 and Figure 73 is corrected by means of controllers designed according to the Theorem 25 and Algorithm 1. The input and initial condition remain as in the simulation of the section 5.3.1. The nonlinearities for Lurie type system in the design of the controllers are of the kind $f_{i} \in F_{(0,2]}, i=1, \ldots, 12$, where 2 is the parameter for the variation of the parametric uncertainties. Note that the Theorem 25 refers to the system (1.2), so should insert the parameters $A, B$ and $C$ obtained from the Hopfield's network of the computational model of the disease according to the equation (5.4). In this case one must 
assume:

$$
\begin{gathered}
A=\operatorname{diag}[-1, \ldots,-1]_{(12 \times 12)}, \quad B=T, \\
\tilde{\Delta}=\left[\begin{array}{ccccc}
\delta_{1} & & & \\
& \ddots & & 0 & \\
& & \delta_{12} & & \\
& 0 & & \delta_{13} & \delta_{14} \\
& & & \delta_{15} & \delta_{16}
\end{array}\right], \quad C=\left[\begin{array}{cc}
0 & 1 \\
1 & 0
\end{array}\right]_{(12 \times 12)} .
\end{gathered}
$$

Remark 22. In the $\tilde{\Delta}$ matrix one has the nonlinearities $f$ of the Lurie type system replaced by the uncertainties $\delta_{1}, \ldots, \delta_{12}$, and the uncertainties $\delta_{13}, \delta_{14}, \delta_{15}$ and $\delta_{16}$ are used to ensure robustness of performance (see (SKOGESTAD; POSTLETHWAITE, 2007) for more information).

Remark 23. After the design of the controllers, the simulation is performed in the Simulink $^{\circledR}$ (via ode 45 Dormand-Prince method with variable-step) using the nonlinearities of the network (5.4), that is, the hyperbolic tangent. The weight matrix is $T=0.5 T_{0}$.

In the first iteration, $m(j \omega)=1.000$ was found. Figure 77 shows the upper bound of $\mu$ reaching 1 . This value of $\mu$ does not satisfy the condition (4.31).

Figure 77: Condition (4.31) not satisfied.

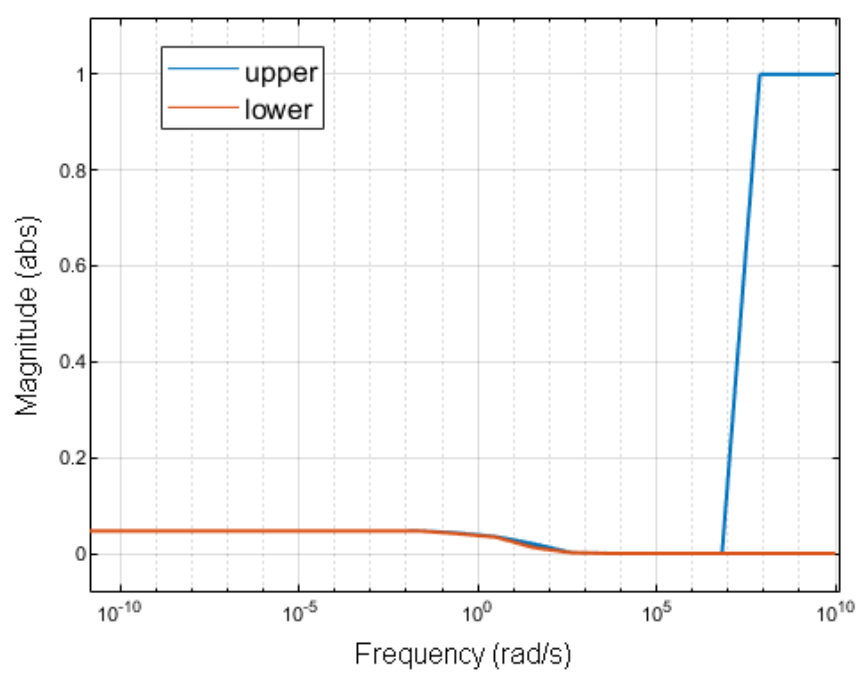

Source: Author.

In the second iteration, as shown in Figure $78, m(j \omega)=0.0067$ was obtained. This value satisfies the condition (4.31) of Theorem 25. Therefore, one has a matrix of controllers, $K=\operatorname{diag}\left[K_{1}(s), \ldots, K_{12}(s)\right]$, that guarantee robustness of stability and performance for the network (5.4). 
Figure 78: Value of $\mu$ that satisfies the condition (4.31) of Theorem 25 .

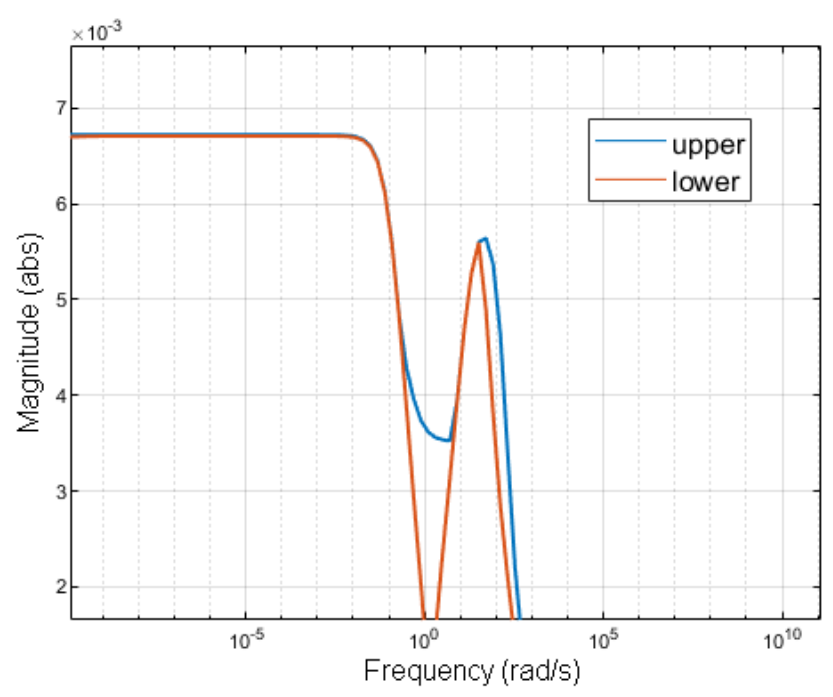

Source: Author.

Applying the controllers designed in the network, one has in Figure 79 the temporal response following the input. One can observe that the response is very fast in this case and the transient is not observable. Finally, according to Figure 80, it is observed that the network memory has been restored.

Figure 79: Temporal response of the network with controllers.

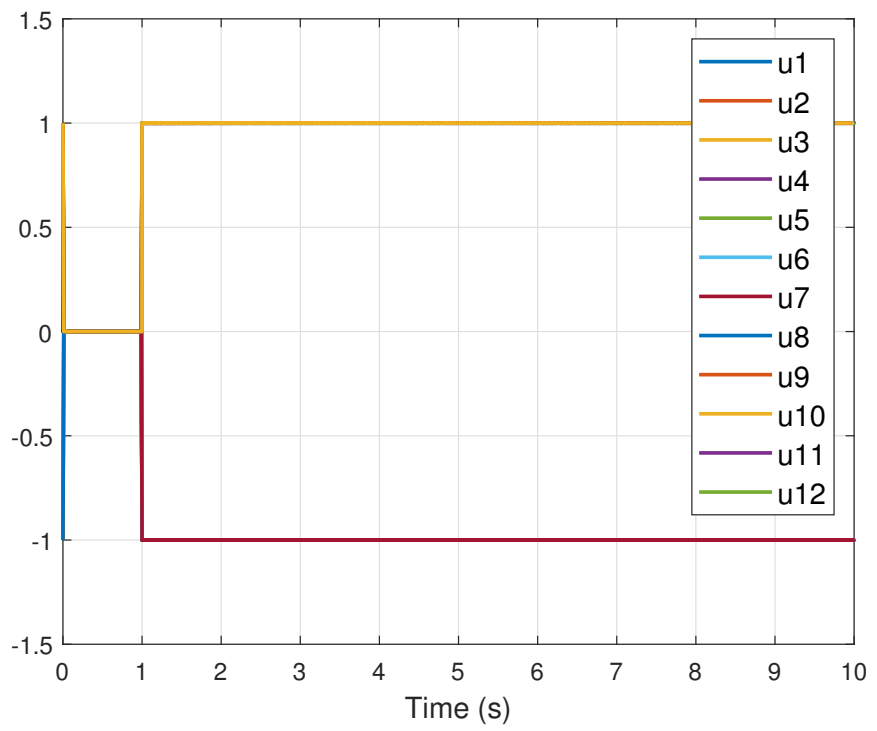

Source: Author. 
Figure 80: Temporal evolution of the letter L in digital sign for network controlled.
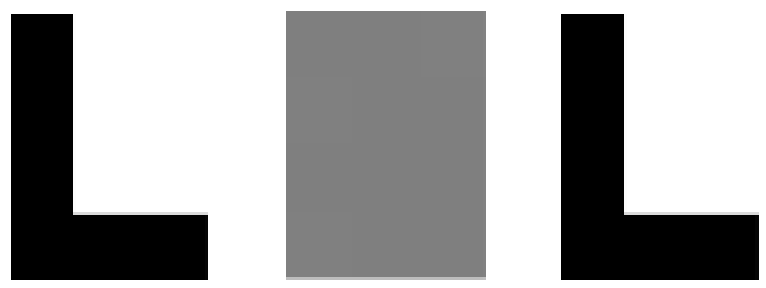

Source: Author.

For networks with serious memory problems, as in Figure 75 , it was not possible to synthesize a controller to correct the problem of memory loss. 


\section{CONCLUSION}

This is a multidisciplinary work, because it presented a vast range of subjects, but with high mathematical rigor, many examples and an unprecedented application. The work starts treating a problem from mechanical engineering (automatic control of aircraft), visits areas of electrical engineering, artificial intelligence and ends with an interesting application in the medical area. The Introduction was conducted starting with the presentation of Lurie problem in a simple and detailed language in relation to that normally found in the literature. The Theoretical Background covers topics such as the classical methods of absolute stability analysis, as well as a new approach to the relationship between absolute stability and robust stability was presented. Furthermore, a new way of transforming nonlinearity into parametric uncertainty was presented, and then the theory of LFT was reformulated specifically for the treatment of the Lurie problem of this work. Still on the Theoretical Background the $\mu$ theory was presented for use in the Lurie problem and for possible extensions in the area of robust control. New conditions for the Lurie problem were developed, new methodologies for design of controllers and a new application were made. The main innovation that this thesis provided, was in the part of systems with delay, where a new technique was built, which presented better results in relation to existing techniques in the literature. The following is a brief discussion and summary of the results obtained through this work and suggestions for future research.

\subsection{Discussion and Summary of the Results}

In relation to continuous SISO systems, it was present a different way to verify the absolute stability of a class of SISO Lurie type systems with the obtaining of new sufficient conditions. Moreover, a condition for time-delay system was obtained and a technique for robust stabilizing controller design via $\mathcal{H}_{\infty}$ Mixed-Sensitivity $(\mathrm{S} / \mathrm{KS} / \mathrm{T})$ was developed, which also solves the performance problem of a Lurie type system in the SISO case. In comparison to other results in the literature, it was found that the condition of the Theorems 13 and 14 are less conservative. In addition, the designed controller is better 
than others in the literature, showing the effectiveness of the presented technique. In relation to discrete SISO systems it was developed two procedures for obtaining robust discrete controllers for a class of SISO Lurie type systems. The controller designed via LMI with polytopic uncertainties presented non-removable error, however, the problem can be easily solved with an integrator. Regarding the controllers via mixed-sensitivity, the controller $(S / K S)$ presented a smooth oscillation in permanent regime, which cannot be removed. The controller $(S / K S / T)$ showed no significant oscillation or error, being, in our analysis, the one that went best for the example proposed. Like in (PINHEIRO; COLÓN, 2017) a theoretical robustness analysis could also be performed. This could prove stability and performance robustness in any frequency band. It is, therefore, a suggestion for a next work.

For the MIMO case, it was presented a different way to verify the absolute stability of some classes of MIMO Lurie type systems. Examples of applications were presented in the absolute stability analysis of complex networks and the synthesis of controllers were possible by means of the theory developed. In comparison with other result in literature (HADDAD; BERNSTEIN, 1993), the thesis method is equivalent in conservatism, but in other, the results of this work are better. Also, our results have the advantage of requiring fewer verifications for a conclusion on absolute stability and are applicable to a wider class of nonlinearities. It was also analyzed time-delay Lurie type systems, and two new condition was proposed, indicating that the theory presented can be extended to systems with time-delay. It was also demonstrated via Chua's circuit that the condition of the Theorem 21 in this thesis is less conservative than others found in the literature (GAO; DU; LIU, 2017; HE et al., 2005; LIU et al., 2010). Furthermore, it was presented a result to analyze robustness of stability and performance of a MIMO Lurie type system in closed-loop. This result, together with Algorithm 1, enabled the synthesis of controllers via DK-Iteration to Lurie type complex network, in particular to Hopfield neural networks.

In applications, one of the consequences of Alzheimer's disease, memory failure, is modeled through the Hopfield's network. In this modeling, it can be seen that the synaptic connection strength between neurons in a biological network is inverse in the modeled artificial neural network. That is, a high weight in the artificial network models lower synaptic interactions between biological neurons, causing memory loss. In order to correct the problem of memory loss in the artificial network, a controller via $\mathcal{H}_{\infty}$ control techniques was designed using a recent theorem from the literature on Lurie's problem. Simulations showed the effectiveness of the model and controller. In the process of healing the artificial network using the controller, it was found that for a high degree of memory loss, the 
controller cannot restore memory. This makes sense when observing a biological network, because it can mean the complete loss of synapses or the death of the neuron. Thus, for practical purposes for Alzheimer's disease, it is supposed that energy inputs in neurons not completely impaired may lead to attenuation in memory loss. This idea is in agreement with the discovery in (THURAISINGHAM, 2015). In this work, the model suggests that the external stimuli in the brain will slow down this memory loss. Nevertheless, if the neuronal connections are already poor, then the enhancements will not be noticeable.

\subsection{Suggestions for Future Research}

For future research, the following items can be considered:

\section{SISO Systems:}

- Extend the results presented in this work to other classes of systems, such as discretetime domain version;

- It is suggested to apply this theory in deep learning, in solving optimization problems, and in complex networks.

\section{MIMO Systems:}

- Extend the results presented in this work to other classes of systems, such as timevarying time-delay MIMO Lurie type systems, and discrete-time domain version;

- Search for explicit conditions from Theorems 18 and 19, like in Theorem 2. That is, to provide conditions with direct values for matrices $A, B$ and $C$ of Lurie type systems (1.2).

- Search for further results in complex networks that could be used in distributed control and filtering, using for example graph theory (DING; WANG; HAN, 2020).

- Apply the theory to artificial neural networks that could be used in deep learning and in solving optimization problems. In fact, the neural approach for solving optimization problems has attracted considerable attention in recent years. The main drawback is that spurious suboptimal responses, due to the existence of many stable equilibrium points, severely limit the performance. The main idea is to train the network respecting the absolute stability criterion in order to avoid the presence of spurious responses and to 
guarantee convergence toward the global optimal solution (LIAO; YU, 2008).

- Explore applications in chaos and fractional derivatives.

- Study bifurcations in the Hopfield network model.

- Investigate the possibility of applying the theory developed in this work to critical Lurie type systems and furthermore research the performance of a Lurie type system in conjunction with phase-locked loop (PIQUEIRA, 2020).

- Model Alzheimer's disease using other types of networks, which include delay, other nonlinearities, and the application of other techniques such as adaptive control using only state feedback, which would represent new synapses. In addition, other control techniques can be explored, such as, the use of the LPV framework with the discrete-time domain and integral quadratic constraints IQC's.

- Use the theory of controller design presented in this thesis to develop robust controllers for power take-off (PTO) of wave energy converters (WEC). It is observed in works such as (GASPAR et al., 2016) and (GASPAR et al., 2021) the necessity of robust controllers, once these systems have many nonlinearities coming from actuators, valves, accumulators, temperature variations, and several sea conditions. Many of these nonlinearities can be considered as the nonlinearities of Lurie type systems. 


\section{REFERENCES}

ABTAHI, S. F.; YAZDI, E. A. Robust control synthesis using coefficient diagram method and $\mu$-analysis: an aerospace example. International Journal of Dynamics and Control, v. 7, p. $595-606,2019$.

AIZERMAN, M. A. On the effect of nonlinear functions of several variables on the stability of automatic control systems (in Russian). Autom. i Telemekh, v. 8, n. 1, p. 20-29, 1947.

AL-SHAYEA, Q. Artificial neural networks in medical diagnosis. Intern. J. Comp. Sci., v. 8, n. 2, 2011.

ALVERGUE, L.; GU, G.; ACHARYA, S. A generalized sector-bound approach to feedback stabilization of nonlinear control systems. International Journal of Robust and Nonlinear Control, v. 23, n. 14, p. 1563-1580, 2013.

ALZHEIMER, A. Uber eine eigenartige erkrankung der hirnrinde (in german). Allg. Zeitschrift Psychiatrie und phychish-Gerichtliche Medizin, v. 64, p. 146-148, 1907.

AOUITI, C.; LI, X.; MIAADI, F. Finite-time stabilization of uncertain delayed-Hopfield neural networks with a time-varying leakage delay via non-chattering control. Sci. China Technol. Sci., v. 62, p. 1111-1122, 2019.

BAKER, G.; GRAVE-MORRIS, P. R. Padé Approximants. [S.l.]: Addison-Wesley Publishing Company, Massachusetts, 1981.

BERNSTEIN, D. S.; HADDAD, W. M.; SPARKS, A. G. A Popov criterion for uncertain linear multivariable systems. Automatica, v. 31, n. 7, p. 1061-1064, 1995.

BIANCHI, F. D.; BATTISTA, H. D.; MANTZ, R. J. Wind Turbine Control Systems. [S.l.]: Springer-Verlag, London, 1981.

BOCCALETTI, S. et al. Complex networks: Structure and dynamics. Physics Reports, v. 424, n. 4, p. 175-308, 2006.

BRAGA, A. P.; CARVAlho, A. C. P. L.; LUDEMIR, T. B. Redes Neurais Artificiais: Teoria e Aplicacões. [S.l.]: LTC, RJ, 2005.

BRAGA, M. F. et al. A new procedure for discretization and state feedback control of uncertain linear systems. In: 52nd IEEE Conference on Decision and Control. [S.l.: s.n.], 2013. p. 6397-6402.

CARRASCO, J.; TURNER, M. C.; HEATH, W. P. Zames-Falb multipliers for absolute stability: From O'Shea's contribution to convex searches. European Journal of Control, v. 28, p. 1-19, 2016. 
CHENG, T. M.; SAVKIN, A. V. Output feedback stabilization of nonlinear networked control systems with non-decreasing nonlinearities: a matrix inequalities approach. International Journal of Robust and Nonlinear Control, v. 17, n. 5-6, p. 387-404, 2007.

DING, D.; WANG, Z.; HAN, Q.-L. A set-membership approach to event-triggered filtering for general nonlinear systems over sensor networks. IEEE Transactions on Automatic Control, v. 65, n. 4, p. 1792-1799, 2020.

DOYLE, J. Analysis of feedback systems with structured uncertainties. IEE Proceedings D (Control Theory and Applications), v. 129, p. 242-250(8), 1982.

DOYLE, J. ONR/Honeywell workshop on advances on multivariable control. [S.l.]: Lecture Notes, Minneapolis, 1984.

DOYLE, J. Structured uncertainty in control system design. In: 24th IEEE Conference on Decision and Control. [S.1.: s.n.], 1985. p. 260-265.

DUAN, W. et al. Improved sufficient lmi conditions for the robust stability of time-delayed neutral-type Lur'e systems. Int. J. Control Autom. Syst., v. 16, p. 2343-2353, 2018.

DUllerud, G. E.; PAGANINI, F. G. A Course in Robust Control Theory: A Convex Approach. [S.1.]: Springer Science and Business Media, New York, 2013.

FEINBERG, E. A.; KASYANOV, P. O.; VOORNEVELD, M. Berge's maximum theorem for noncompact image sets. Journal of Mathematical Analysis and Applications, v. 413, n. 2, p. 1040-1046, 2014.

FILLIT, H.; CUMMINGS, J. Practice guidelines for the diagnosis and treatment of Alzheimer's disease in a managed care setting: Part II-Pharmacologic therapy. Alzheimer's disease (AD) Managed care advisory council. Manag Care Interface, p. 51-56, 2000.

FORTI, M. et al. On absolute stability of neural networks. In: Proceedings of IEEE International Symposium on Circuits and Systems - ISCAS '94. [S.1.: s.n.], 1994. v. 6, p. 241-244.

FREITAS, E. V. Tratado de Geriatra e Gerontologia. [S.l.]: Guanabara Koogan 2nd edn., Rio de Janeiro, 2006.

GAO, Q.; DU, J.; LIU, X. An improved absolute stability criterion for time-delay Lur'e systems and its frequency domain interpretation. Circuits Syst Signal Process, v. 36, p. 916-930, 2017.

GAO, Y.; YANG, X.; SHARDT, Y. A. Robust decoupling mixed sensitivity controller design of looper control system for hot strip mill process. Advances in Mechanical Engineering, v. 10, n. 11, p. 1-10, 2018.

GAPSKI, P. B.; GEROMEL, J. C. A convex approach to the absolute stability problem. IEEE Trans. Automat. Contr., v. 25, p. 613-617, 1994.

GASPAR, J. F. et al. Speed control of oil-hydraulic power take-off system for oscillating body type wave energy converters. Renewable Energy, v. 97, p. 769-783, 2016. 
GASPAR, J. F. et al. Hydraulic power take-off concept for the $\mathrm{m} 4$ wave energy converter. Applied Ocean Research, v. 106, p. 102462, 2021.

GEROMEL, J. C.; KOROGUI, R. H. Controle Linear de Sistemas Dinâmicos. [S.1.]: Editora Blucher, São Paulo, 2011.

GIL, D. et al. Application of artificial neural networks in the diagnosis of urological dysfunctions. Expert Systems with Applications, v. 36, n. 3, Part 2, p. 5754-5760, 2009.

GU, D. W.; PETKOV, P. H.; KOnSTANTINOV, M. M. Robust Control Design with MATLAB, in: Series Advanced Textbooks in Control and Signal Processing. [S.1.]: Springer, London, 2006.

GUTZWILLER, J. Das Herz, etwas zu wagen. [S.l.]: Friedrich Bahn Verlag: Neukirchen-Vluyn, ISBN: 3761593031, 2000.

GUZMAN, M. Ecuaciones Diferenciales Ordinarias - Teoria de Estabilidad y Control. [S.l.]: Alhambra, Madrid, 1980.

GYURKOVICS, E.; ESZES, D. Sufficient conditions for stability and stabilization of networked control systems with uncertainties and nonlinearities. International Journal of Robust and Nonlinear Control, v. 25, n. 16, p. 3004-3022, 2015.

HADDAD, W. M.; BERNSTEIN, D. S. Explicit construction of quadratic Lyapunov functions for the small gain, positivity, circle, and Popov theorems and their application to robust stability. part I: Continuous-time theory. International Journal of Robust and Nonlinear Control, v. 3, n. 4, p. 313-339, 1993.

HAKE, A. M. The treatment of Alzheimer's disease: The approach from a clinical specialist in the trenches. Seminars In Neurology, v. 22, n. 1, p. 071-074, 2002.

HAO, F.; ZHAO, X. Absolute stability of Lurie networked control systems. International Journal of Robust and Nonlinear Control, v. 20, n. 12, p. 1326-1337, 2010.

HE, Y. et al. Robust stability for delay Lur'e control systems with multiple nonlinearities. Journal of Computational and Applied Mathematics, v. 176, n. 2, p. 371-380, 2005.

HETEL, L.; DAAFOUZ, J.; IUNG, C. LMI control design for a class of exponential uncertain systems with application to network controlled switched systems. In: 2007 American Control Conference. [S.l.: s.n.], 2007. p. 1401-1406.

HOPFIELD, J. J. Neurons with graded response have collective computational properties like those of two-state neurons. Proceedings of the National Academy of Sciences, National Academy of Sciences, v. 81, n. 10, p. 3088-3092, 1984.

HUANG, Y.-P.; ZHOU, K. Robust stability of uncertain time-delay systems. IEEE Transactions on Automatic Control, v. 45, n. 11, p. 2169-2173, 2000.

IMANI, A. A multi-loop switching controller for aircraft gas turbine engine with stability proof. International Journal of Control, Automation and Systems, v. 17, p. 1359-1368, 2019. 
IYERNGAR, V.; BALAGANI, K. The use of artifical neural networks to study and improve neural synaptic promoters and connectivity in Alzheimer's disease states. In: IEEE. IEEE 4th Intern. Conference on Intelligent Syst. Design and Application. [S.1.], 2004. p. 525-530.

KALMAN, R. E. Liapunov functions for the problem of Lurie in automatic control. Proc. Natl. Acad. Sci., v. 49, n. 8, p. 201-205, 1963.

KASZKUREWICZ, E.; BHAYA, A. Comments on "necessary and sufficient condition for absolute stability of neural networks". IEEE Transactions on Circuits and Systems I: Fundamental Theory and Applications, v. 42, n. 8, p. 497-499, 1995.

KAZEMY, A.; FARROKHI, M. Synchronization of chaotic Lur'e systems with state and transmission line time delay: a linear matrix inequality approach. Transactions of the Institute of Measurement and Control, v. 39, n. 11, p. 1703-1709, 2017.

KHALIL, H. K. Nonlinear Systems. [S.l.]: Prentice Hall, third edition, New Jersey, 2002.

KRASOVSKII, N. N. On the stability of the solutions of a system of two differential equations (in Russian). Prikl. Mat. i Mekh., v. 17, p. 651-672, 1953.

LANCASTER, P.; TISMENETSKY, M. Multivariable feedback control: analysis and design. [S.l.]: Academic Press. San Diego, CA, 1985.

LEE, C. M.; JUANG, J. C. A novel approach to stability analysis of multivariable Lurie systems. In: IEEE. IEEE International Conference on Mechatronics and Automation, Niagara Falls, Ont. [S.l.], 2005. p. 199-203.

LI, X. et al. Instability and unboundedness analysis for impulsive differential systems with applications to Lurie control systems. Int. J. Control Autom. Syst., v. 16, p. 1521-1531, 2018.

LIAO, X. Absolute Stability of Nonlinear Control Systems. [S.l.]: Kluwer Academic China Science Press, Beijing, 1993.

LIAO, X.; WANG, L.; YU, P. Stability of Dynamical Systems. [S.l.]: Elsevier, 2007.

LIAO, X.; YU, P. Absolute Stability of Nonlinear Control Systems. [S.l.]: Springer, 2nd edition, Netherlands, 2008.

LIAO, X. et al. Robust absolute stability of Lurie interval control systems. Int. J. Robust Nonlinear Control, v. 17, n. 18, p. 1669-1689, 2007.

LIN, H.; WANG, C.; TAN, Y. Hidden extreme multistability with hyperchaos and transient chaos in a Hopfield neural network affected by electromagnetic radiation. Nonlinear Dyn., v. 99, p. 2369-2386, 2020.

LIU, X. et al. New absolute stability criteria for time-delay Lur'e systems with sector-bounded nonlinearity. International Journal of Robust and Nonlinear Control, v. 20, n. 6, p. 659-672, 2010.

LURIE, A. I.; POSTNIKOV, V. N. On the theory of stability of control systems (in Russian). Prikl. Mat. i Mekh., v. 8, n. 3, p. 246-248, 1944. 
MATSUMOTO, T. A chaotic attractor from Chua's circuit. IEEE Transactions on Circuits and Systems, v. 31, n. 12, p. 1055-1058, 1984.

MCKHANN, G. et al. Clinical diagnosis of Alzheimer's disease: report of the nincds-adrda work group under the auspices of department of health and human services task force on Alzheimer's disease. Neurology, v. 34, n. 7, p. 939-944, 1984.

MEYER, C. D. Matrix Analysis and Applied Linear Algebra. [S.l.]: SIAM, Philadelphia, 2000 .

MONTEIRO, L. H. A. Sistemas Dinâmicos. [S.l.]: Livraria da Física 2nd edn., São Paulo, 2006.

MORRISON, M.; MAIA, P. D.; KUTZ, J. N. Preventing neurodegenerative memory loss in Hopfield neuronal networks using cerebral organoids or external microelectronics. Computational and Mathematical Methods in Medicine, n. Article ID 6102494, p. 13 pages, 2017.

MORTON, B. G.; MCAFOOS, R. M. A mu-test for robustness analysis of a realparameter variation problem. In: 1985 American Control Conference. [S.l.: s.n.], 1985. p. $135-138$.

NADERI, T. et al. Revisiting Kalman and Aizerman conjectures via a graphical interpretation. IEEE Transactions on Automatic Control, v. 64, n. 2, p. 670-682, 2019.

OGATA, K. Modern Control Engineering. [S.l.]: Prentice Hall, New Jersey, 2010.

OLIVEIRA, M. C.; GEROMEL, J. C.; BERNUSSOU, J. Extended h 2 and h norm characterizations and controller parametrizations for discrete-time systems. International Journal of Control, v. 75, n. 9, p. 666-679, 2002.

PEIRAN, L. et al. $\mathcal{H}_{\infty}$ control synthesis for Lurie networked control systems with multiple delays based on the non-uniform characteristic. Asian Journal of Control, v. 15, n. 4, p. 1112-1123, 2012.

PINHEIRO, R. F. The Lurie problem and applications to neural networks (in Portuguese). [S.l.]: Master's thesis, USP, São Paulo, 2015.

PINHEIRO, R. F.; COLÓN, D. Controller by $\mathcal{H}_{\infty}$ mixed-sensitivity design $(\mathrm{S} / \mathrm{KS} / \mathrm{T})$ for Lurie type systems. In: ABCM. 24th ABCM International Congress of Mechanical Engineering, Curitiba. [S.l.], 2017.

PINHEIRO, R. F.; COLÓN, D. An application of the Lurie problem in Hopfield neural networks. In: SPRINGER. Proceedings of DINAME 2017. [S.1.], 2019. p. 371-382.

PINHEIRO, R. F.; COLÓN, D. Analysis and synthesis of single-input-single-output Lurie type systems via $\mathcal{H}_{\infty}$ mixed-sensitivity. Transactions of the Institute of Measurement and Control, 2021.

PINHEIRO, R. F.; COLÓN, D. On the $\mu$-analysis and synthesis of mimo Lurie type systems with application in complex networks. Circuits, Systems, and Signal Processing, v. 40, p. 193-232, 2021. 
PIQUEIRA, J. R. C. Delay-free phase-locked loop ring: Equilibrium and phase perturbation. Physica D: Nonlinear Phenomena, v. 404, p. 132344, 2020. ISSN 0167-2789.

PLISS, V. A. Aizerman problem for dimension 3 (in Russian). Dokl. Akad. Nauk., Tom 17, n. 3, 1958.

POPOV, V. M. Absolute stability of nonlinear systems of automatic control. Remote Control, v. 22, n. 8, p. 857-875, 1961.

QI, X.; XIA, J.; AL. et. On the robust stability of active disturbance rejection control for siso systems. Circuits, Systems, and Signal Processing, v. 36, p. 65-81, 2017.

RASVAN, V. Systems with monotone and slope restricted nonlinearities. Tatra Mt. Math. Publ, v. 48, p. 165-187, 2011.

RASVAN, V.; POPESCU, D. On the problem of aizerman. In: 2012 16th International Conference on System Theory, Control and Computing (ICSTCC). [S.l.: s.n.], 2012. p. $1-6$.

SANJEEWA, S. D.; PARNICHKUN, M. Control of rotary double inverted pendulum system using mixed sensitivity $H_{\infty}$ controller. International Journal of Advanced Robotic Systems, v. 16, n. 2, p. 1012-1017, 2019.

SCHEFF, S. W. et al. Synaptic alterations in CA1 in mild Alzheimer disease and mild cognitive impairment. Neurology, v. 68, n. 18, p. 1501-1508, 2007.

SELKOE, D. J. Alzheimer's disease: Genes, proteins, and therapy. Physiological Reviews, v. 81, n. 2, p. 741-766, 2001.

SERGiO, A. T.; BRAGA, D. S.; BUARQUE, L. N. F. Computational modeling of Alzheimer's disease symptoms using Venn's network. In: ABRICOM/SBIC, OURO PRETO. CBRN 2009 - Anais do IX Congresso Brasileiro de Redes Neurais. [S.l.], 2009.

SERON, M.; DONA, J. On invariant sets and closed-loop boundedness of Lurie - type nonlinear systems by LPV-embedding. Int. J. Robust Nonlinear Control, v. 26, n. 5, p. 1092-1111, 2015.

SHIEH, L. S.; WANG, W.; CHEN, G. Discretization of cascaded continuous-time controllers and uncertain systems. Circuits, Systems and Signal Processing, v. 17, n. 5, p. 591-611, 1998.

SKOGESTAD, S.; POSTLETHWAITE, I. Multivariable feedback control: analysis and design. [S.l.]: John Wiley and Sons, 2nd-edn., New York, 2007.

SLOTINE, J. J.; LI, W. Applied Nonlinear Control. [S.l.]: Prentice-Hall, New Jersey, 1991.

SWIETLIK, D. et al. Computer model of synapse loss during an Alzheimer's disease-like pathology in hippocampal subregions DG, CA3 and CA1 - the way to chaos and information transfer. Entropy, v. 21, p. 408, 2020. 
TABEKOUENG, N. Z.; KENGNE, J.; FOTSIN, H. B. Coexistence of multiple stable states and bursting oscillations in a 4d Hopfield neural network. Circuits Syst Signal Process, v. 39, p. 3424-3444, 2020.

TAN, N.; ATHERTON, D. P. Robustness analysis of control systems with mixed perturbations. Transactions of the Institute of Measurement and Control, v. 25, n. 2, p. 163-184, 2003.

TERRY, R. D. Cell death or synaptic loss in Alzheimer disease. Journal of Neuropathology and Experimental Neurology, v. 59, n. 12, p. 1118-1119, 2000.

THURAISINGHAM, R. A. Dementia and Hopfield model. J. Neural Transm., v. 122, p. 773-777, 2015.

TOWNLEY, A. et al. Existence and learning of oscillations in recurrent neural networks. IEEE Trans. Neural Networks, v. 11, p. 205-214, 2000.

VANANTWERP, J. G.; BRAATZ, R. D. A tutorial on linear and bilinear matrix inequalities. Journal of Process Control, v. 10, n. 4, p. 363-385, 2000.

VIDYASAGAR, M. Nonlinear Systems Analysis. [S.l.]: Prentice Hall, 2nd. edn., New Jersey, 1993. v. 2.

WANG, Y.; XUE, Y.; ZHANG, X. Less conservative robust absolute stability criteria for uncertain neutral-type Lur'e systems with time-varying delays. Journal of the Franklin Institute, v. 353, n. 4, p. 816-833, 2016.

WANG, Z.; LUNDSTROM, P.; SKOGESTAD, S. Representation of uncertain time delays in the $H_{\infty}$ framework. International Journal of Control, v. 59, n. 3, p. 627-638, 1994.

YI, Z.; HENG, P. A.; VADAKKEPAT, P. Absolute periodicity and absolute stability of delayed neural networks. IEEE Transactions on Circuits and Systems I: Fundamental Theory and Applications, v. 49, n. 2, p. 256-261, 2002.

YU, X.; LIAO, F. Preview tracking control for discrete-time nonlinear Lur'e systems with sector-bounded nonlinearities. Transactions of the Institute of Measurement and Control, v. 41, n. 10, p. 2726-2737, 2019.

ZAK, S. H. Systems and Control. [S.1.]: Oxford University Press, New York, 2003.

ZAMES, G. On the input-output stability of time-varying nonlinear feedback systemspart II: Conditions involving circles in the frequency plane and sector nonlinearities. IEEE Transactions on Automatic Control, v. 11, n. 3, p. 465-476, 1966.

ZENG, H. et al. Improved stabilization method for Lurie networked control systems. IEEE Transactions on Automatic Control, n. Article ID 789398, p. 6 pages, 2014.

ZENG, H.-B. et al. Absolute stability and stabilization for Lurie networked control systems. International Journal of Robust and Nonlinear Control, v. 21, n. 14, p. 1667-1676, 2010.

ZHENG, J.; CUI, B. State estimation of chaotic Lurie systems via communication channel with transmission delay. Circuits Syst Signal Process, v. 37, p. 4568-4583, 2018. 
ZHOU, C. et al. A unified associative memory model based on external inputs of continuous recurrent neural networks. Neurocomputing, v. 186, p. 44-53, 2016.

ZHOU, K.; DOYLE, J. C.; GLOVER, K. Optimal Control. [S.l.]: Prentice Hall, New Jersey, 1995. 


\section{APPENDIX A - A RESULT IN PARAMETRIZED OPTIMIZATION}

In this thesis, there are some optimization problems that depends on a set of parameters $\theta_{1}, \theta_{2}, \cdots, \theta_{p}$ that can be grouped in a vector $\theta$. In those cases, the objective function if of the form $f: X \times \Theta \rightarrow \mathbb{R}$, where $X$ is the set of decision variables (the searched space in order to find the optimal) and $\Theta$ is the parameter space.

The result of the solution of the optimization problem is two functions $x^{*}(\theta)$ and $v(\theta)=\sup _{x \in X} f(x, \theta)$.

Definition 17 (Constrained Optimization). A constrained optimization problem is an optimization problem like:

$$
\max _{x \in X} f(x), \text { subject to } g_{i}(x) \leq 0, \text { for } i=1, \cdots, m \text {. }
$$

Theorem 26 (Berge's Continuous Maximum Theorem). Consider the general constrained maximization problem:

$$
\max _{x \in G(\theta)} f(x, \theta) .
$$

If the objective function $f: X \times \Theta \rightarrow \mathbb{R}$ is continuous and the set function $G: \Theta \rightarrow 2^{X}$ is continuous and compact-valued, then the value function $v: \Theta \rightarrow \mathbb{R}$ :

$$
v(\theta)=\sup _{x \in G(\theta)} f(x, \theta)
$$

is continuous and the set function of optimal values, that is:

$$
x^{*}(\theta)=\operatorname{argmax}_{x \in G(\theta)} f(x, \theta),
$$

is nonempty, compact-valued and upper semicontinuous.

Proof. It can be found in (FEINBERG; KASYANOV; VOORNEVELD, 2014). 